Florida International University FIU Digital Commons

FIU Electronic Theses and Dissertations

University Graduate School

7-24-2017

\title{
Essays on Women's Issues and Economic Development
}

Amy Ramnarine

amyramnarine@gmail.com

DOI: $10.25148 /$ etd.FIDC004010

Follow this and additional works at: https://digitalcommons.fiu.edu/etd

Part of the Growth and Development Commons

\section{Recommended Citation}

Ramnarine, Amy, "Essays on Women's Issues and Economic Development" (2017). FIU Electronic Theses and Dissertations. 3556.

https://digitalcommons.fiu.edu/etd/3556

This work is brought to you for free and open access by the University Graduate School at FIU Digital Commons. It has been accepted for inclusion in FIU Electronic Theses and Dissertations by an authorized administrator of FIU Digital Commons. For more information, please contact dcc@fiu.edu. 


\section{FLORIDA INTERNATIONAL UNIVERSITY}

Miami, Florida

\section{ESSAYS ON WOMEN'S ISSUES AND ECONOMIC DEVELOPMENT}

A dissertation submitted in partial fulfillment of

the requirements for the degree of

DOCTOR OF PHILOSOPHY

in

ECONOMICS

by

Amy Vijaya Ramnarine 
To: Dean John F. Stack Jr.

Green School of International and Public Affairs

This dissertation, written by Amy Vijaya Ramnarine, and entitled Essays on Women's Issues and Economic Development, having been approved in respect to style and intellectual content, is referred to you for judgment.

We have read this dissertation and recommend that it be approved.

$\begin{array}{r}\hline \text { Cem Karayalcin } \\ \hline \text { Alejandro Arrieta } \\ \hline \text { Tobias Pfutze, Co-Major Professor } \\ \hline \text { Mihaela Pintea, Co-Major Professor }\end{array}$

Date of Defense: July 24, 2017

The dissertation of Amy V. Ramnarine is approved.

Dean John F. Stack, Jr

Green School of International and Public Affairs

Andrés G. Gil

Vice President for Research and Economic Development and Dean of the University Graduate School

Florida International University, 2017 
C Copyright 2017 by Amy Vijaya Ramnarine

All rights reserved. 


\section{DEDICATION}

I dedicate this dissertation to my mother and father. Thank you for always bringing me sunshine on dark and cloudy days. Thank you for teaching me never to give up. And thank you, for never giving up on me. 


\section{ACKNOWLEDGMENTS}

I would like to thank my advisor, Dr. Pintea who has helped me in the transition from student to researcher. Her comments, support and encouragement over the past five years has truly been invaluable. I would also like to express my gratitude to Dr. Pfutze for his great insights and for all the time he has invested in helping me through my dissertation. I am deeply grateful to my committee members: Dr. Karayalcin and Dr. Arrieta for their patience, contributions and support. I would also like to express my sincere appreciation for the Economics Department Staff: Mayte, Mariela and Lorette for always addressing every concern and for always providing me with help. 


\section{ABSTRACT OF THE DISSERTATION \\ ESSAYS ON WOMEN'S ISSUES AND ECONOMIC DEVELOPMENT \\ by}

Amy Vijaya Ramnarine

Florida International University, 2017

Miami, Florida

Professor Mihaela Pintea, Major Professor

This dissertation is composed of three essays under the recurring theme of women's issues, child health and economic development. In the first chapter, I examine the effect of Child Marriage on the health outcomes of offspring in Bangladesh. I use an exogenous variation in drought and flood shocks as an instrument for Child Marriage and measure children's health outcomes by stunting, from the height-for-age index. I develop a theoretical model to show that the decision to marry a daughter early is driven by the type of income shocks that a household is exposed to. From the empirical estimation, I find that children from Child Marriage unions are more likely to be stunted. I also find that the effect of Child Marriage is concentrated through increases in severe stunting.

In the second chapter, I estimate the impact of Child Marriage on the mortality outcomes of offspring in Bangladesh. Similar to the empirical strategy in the first chapter, I focus on the effect of an exogenous temporary shock on the marriage decision, to allow for identification of a causal impact. The results indicate that in comparison to children of later-married women, children from Child Marriage unions are more likely to die before reaching the age of five. Further, I find that the effect of Child Marriage is smaller on infant 
mortality. By examining the impact by gender, the results suggest that the predominant effect of Child Marriage is focused on increases in male child and infant mortality. In the third chapter, I examine the effect of Sharia Law on the health outcomes of children in Nigeria. The measures of health outcomes are constructed from the height-for-age, weight-for-age and weight-for-height indices. I apply a difference-in-differences estimation strategy and exploit the variation in exposure to Sharia law across time and state of residence. I also explore the effect of Sharia Law on women's empowerment. The results indicate that Sharia Law substantially increases the probability of stunting in children. In states with high enforcement of Sharia Law, children are more likely to be stunted and underweight. The results suggest a negative association between Sharia Law and women's empowerment with respect to household and medical decision-making, control over resources and self-worth. 


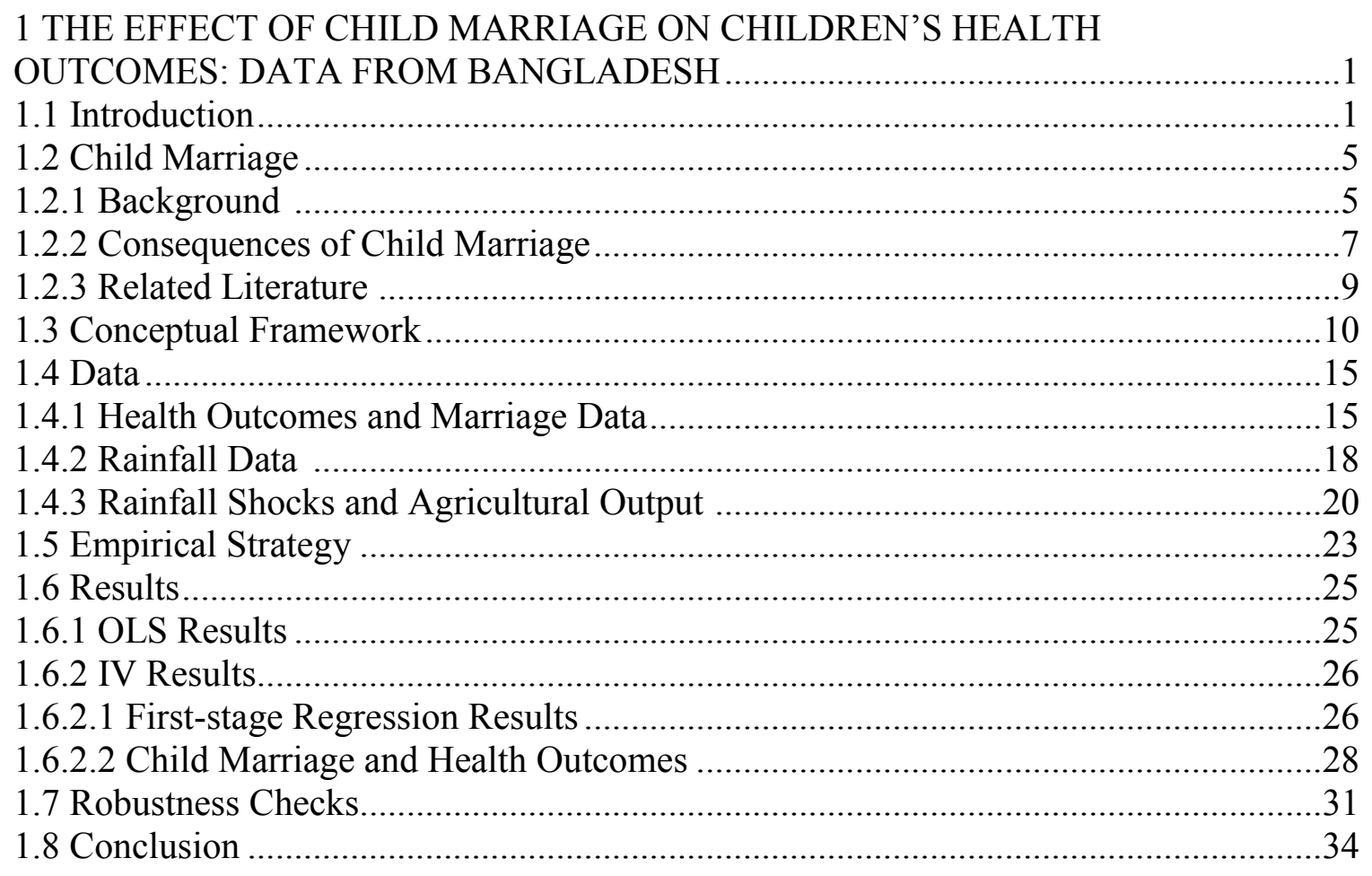

2 CHILD MARRIAGE AND MORTALITY OUTCOMES OF OFFSPRING .................51

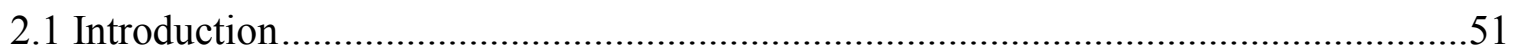

2.2 Channels of Impact of Child Marriage on Offspring's Mortality ................................56

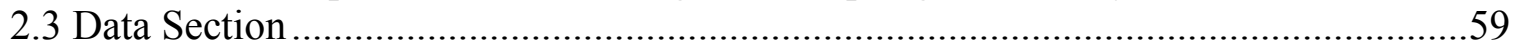

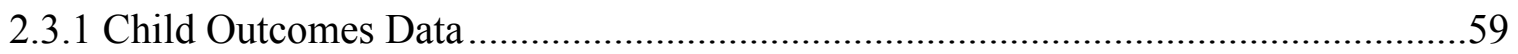

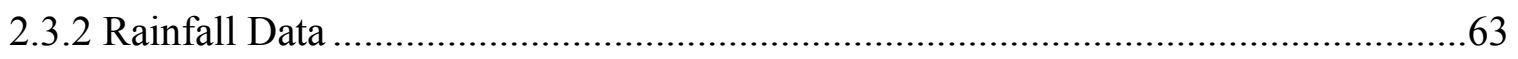

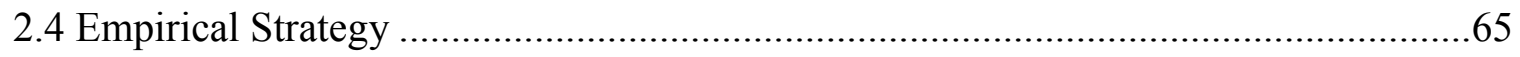

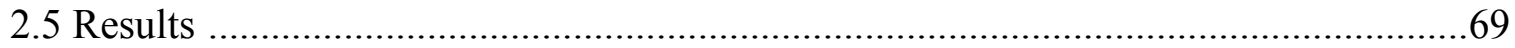

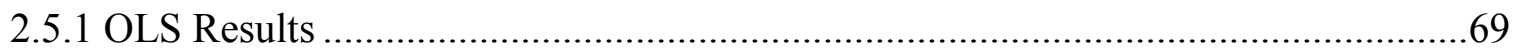

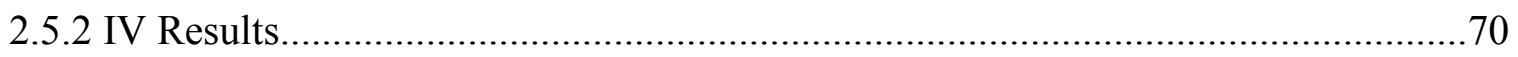

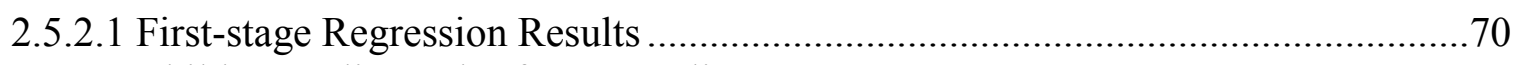

2.5.2.2 Child Mortality and Infant Mortality Outcomes …………..................................71

2.5.2.3 Child and Infant Mortality Outcomes by Gender .................................................74

2.6 Child Marriage and the Status of Women in the Household ........................................76

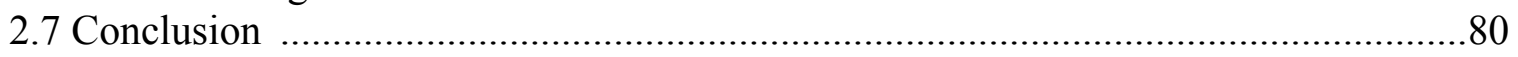

3 SHARIA LAW, CHILDREN'S HEALTH OUTCOMES AND WOMEN'S

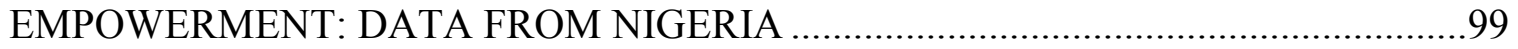

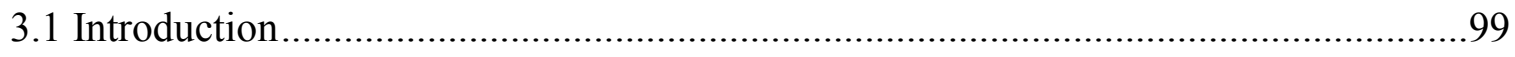




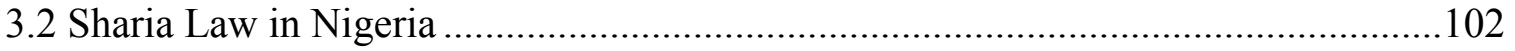

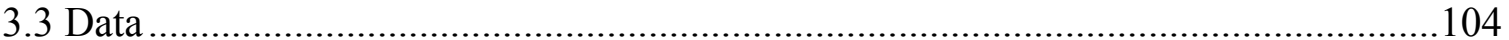

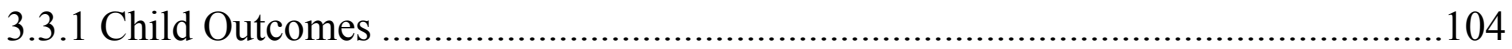

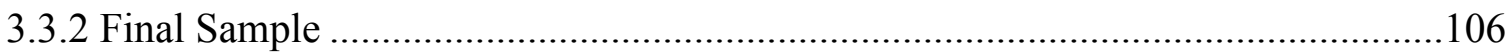

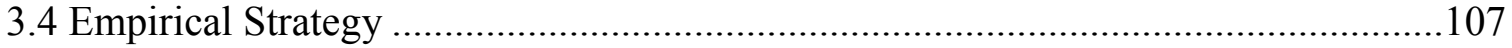

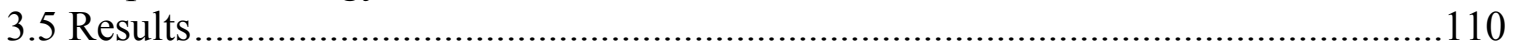

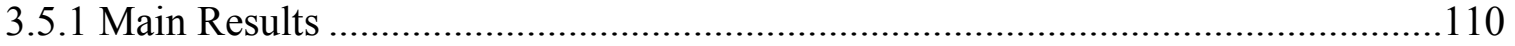

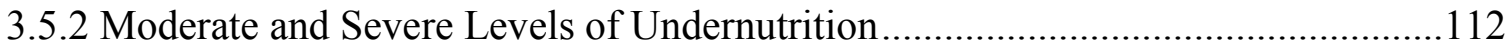

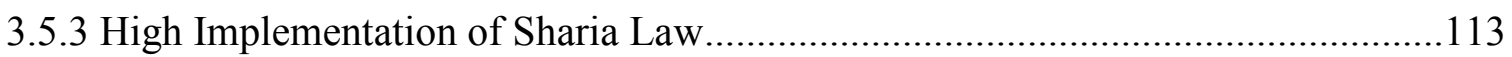

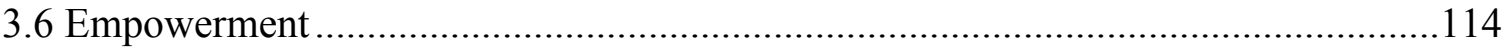

3.6.1 Empowerment and Health Outcomes of Women and Children............................116

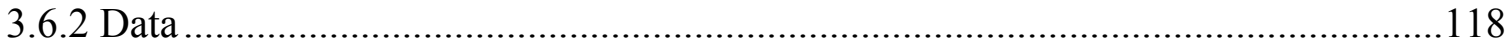

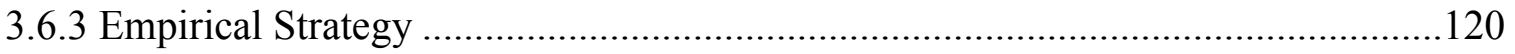

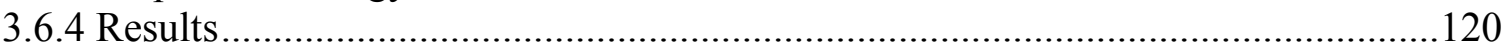

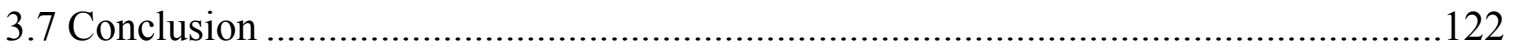

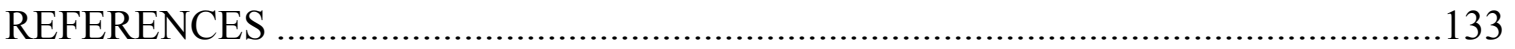

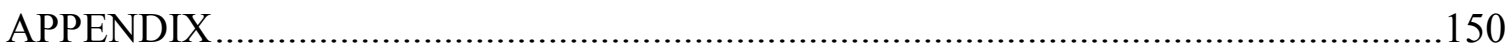

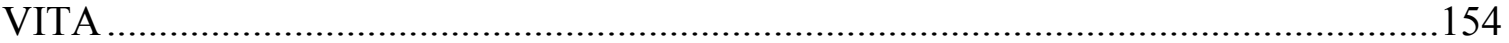




\section{LIST OF TABLES}

TABLE

PAGE

1.1 Summary Statistics. .39

1.2 Effect of Rainfall Shock on Crop Yields .40

1.3 OLS Results - Effect of Child Marriage on Stunting .41

1.4 First-stage IV Results - Effect of Child Marriage on Stunting .42

1.5 IV Results - Effect of Child Marriage on Stunting .44

1.6 First-stage IV Results - Weather Shock Definition .45

1.7 IV Results - Weather Shock Definition .47

1.8 First-stage Results - Choice of Instruments .48

1.9 IV Results - Choice of Instruments .49

1.10 IV Results - Effect on Moderate Stunting and Severe Stunting .50

2.1 Summary Statistics for Child Mortality Sample .................................................86

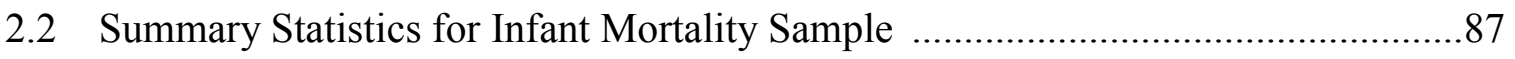

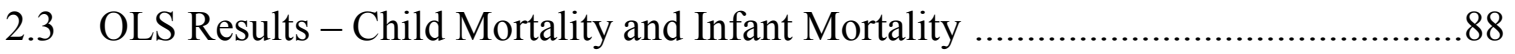

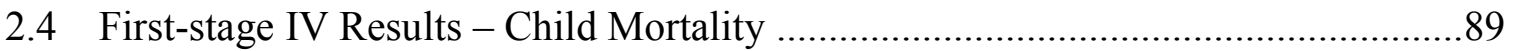

2.5 First-stage IV Results - Infant Mortality ................................................... 91

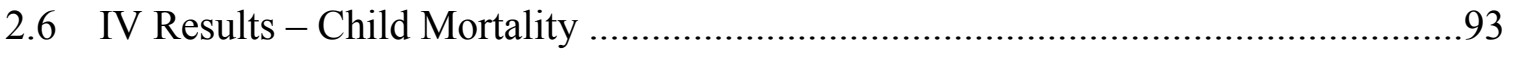

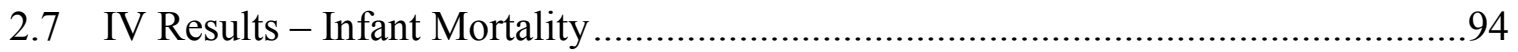

2.8 IV Results - Child Mortality by Gender ......................................................95

2.9 IV Results - Infant Mortality by Gender ....................................................96

2.10 IV Results - Access to Healthcare. .97 


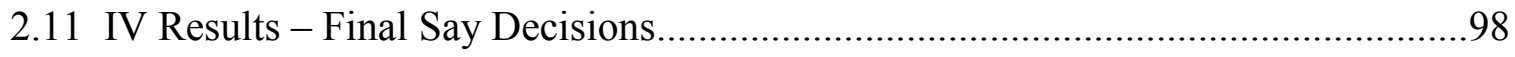

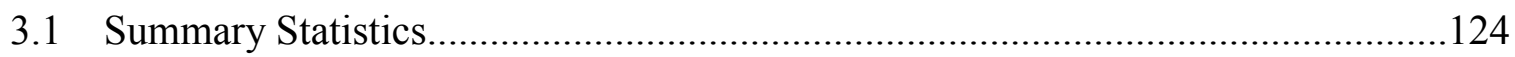

3.2 Effect of Sharia Law on Children's Nutritional Outcomes ....................................125

3.3 Effect of Sharia Law on Moderate and Severe Undernutrition .............................126

3.4 Effect of High Implementation on Children's Nutritional Outcomes ....................128

3.5 Effect of High Implementation on Moderate and Severe Undernutrition .............129

3.6 Effect of Sharia Law on Household Decision-making .........................................130

3.7 Effect of Sharia Law on Medical Decision-making ……………........................131

3.8 Effect of Sharia Law on Attitudes towards Violence ............................................132

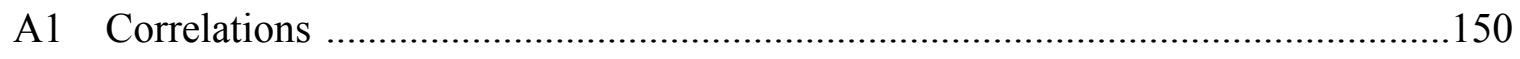

A2 OLS Results - Effect on Moderate Stunting and Severe Stunting .........................151

A3 First-stage IV Results - Child Mortality by Gender ..............................................152

A4 First-stage IV Results - Infant Mortality by Gender ...........................................153 


\section{LIST OF FIGURES}

FIGURES

PAGE

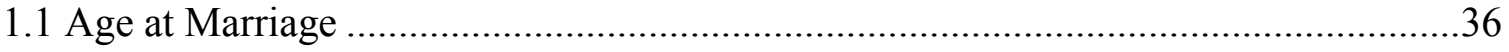

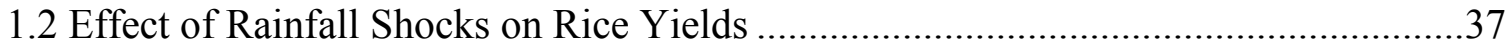

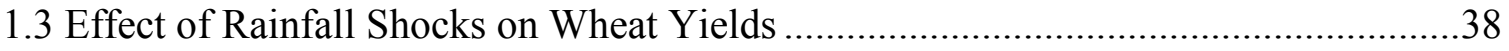

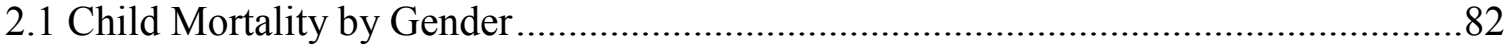

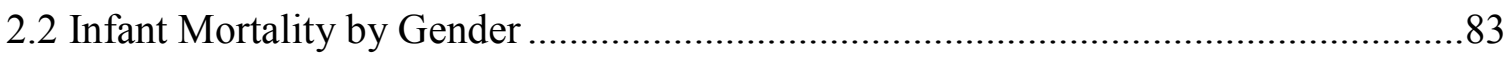

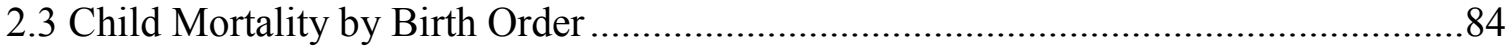

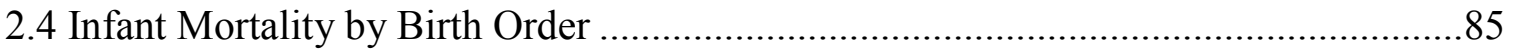




\section{CHAPTER 1}

\section{THE EFFECT OF CHILD MARRIAGE ON CHILDREN'S HEALTH \\ OUTCOMES: DATA FROM BANGLADESH}

\subsection{Introduction}

Child Marriage, defined as a legal or informal union before the age of 18 , is a violation of human rights and a significant barrier to social and economic development. At present, each year an average of 15 million girls become child brides. Numerous programs and initiatives $^{1}$ (Gupta et al. 2008, Lee-Rife et al. 2012, Verma et al. 2013) have been launched to reduce the practice of Child Marriage but the pace of change in reduction is slow. In comparison to the 1980 s where 1 out of 4 women was married before the age of 18 , today 1 out of 3 marries too early (UNICEF 2014). Child Marriage remains prevalent mainly in Sub-Saharan Africa and South Asia. Bangladesh, which I use as the setting, has the highest rate of Child Marriage within the South Asian region and the fourth highest world-wide. The most recent estimates report that 52 percent of females are married before the age of 18, and 18 percent are married by the age of 15 (UNICEF 2016).

Child Marriage has physical, psychological and intellectual implications for the child bride. A large body of evidence has documented the negative consequences, including lower educational attainment, higher susceptibility to violence, poor physical and mental

\footnotetext{
${ }^{1}$ Some examples of programs and initiatives include: USAID's Safe Schools programs, The Yemen Early Marriage Project (YEMP), Maharashtra Life Skills Program in India, Abriendo Oportunidades program in Guatemala, the Gender Roles, Equality and Transformations (GREAT) project, BRAC's Social and Final Empowerment of Adolescents (SoFEA) program in Bangladesh, Save the Children's Kishoree Kontha program in Bangladesh, the Berhane Hewane program in Ethiopia, the Zomba Cash Transfer program in Malawi, the Community-based Rural Livelihood program in Afghanistan, and Oxfam's Integrated Action on Poverty and Early Marriage (IAPE) project in Yemen.
} 
health effects, reduced labor force participation and less power and decision making within the marital household (Parsons et al. 2015, Jensen and Thornton 2003, Malhotra 2013). A priori, these adverse effects should in turn have an impact on the children of the child bride. There is a gap in the literature, however, in assessing the outcomes of children produced from Child Marriage unions. Such an assessment is quite important for policy as it highlights that the effect of Child Marriage may extend into the next generation. It serves as a notification that Child Marriage is not just an issue pertinent to child brides, but is an impediment to their offspring, the future human capital.

In this paper, I provide new evidence on the relationship between Child Marriage and the health outcomes of children produced from these unions. Specifically, using the condition of stunting as a measure of health outcomes, I seek to address the question of whether children from Child Marriage unions suffer poorer health outcomes. Stunting, which is constructed from the height-for-age index, reflects the failure to receive adequate nutrition over a long period and has the advantage of being robust to transitory spells of disease and seasonality in diet. Children that are stunted are subject to poor development outcomes across their life course. Stunting is associated with a higher risk of infection and mortality, impaired psychomotor and cognitive development, and lower educational attainment. These factors transfer to adulthood and diminish work capacity and earnings, affecting labor productivity (Martorell 1997, Black et al. 2013, Rodriguez 2016, Deshmukh, Sinha and Dongre 2013).

To answer the question of whether children's health outcomes are affected by Child Marriage, I employ a two-step methodology where I make use of the exogenous variation in rainfall as an instrumental variable for Child Marriage. Rainfall shocks are an important 
source of income variation in countries like Bangladesh, due largely to the household's dependence on the agricultural sector. I construct two measures of rainfall shocksdrought and flood - and show that they each generate significant but different changes in the household's decision to enter into a Child Marriage union. I explain these differences through the development of a simple model of marriage decisions, in which the potential brides' households are exposed to negative and positive shocks to their income. Households have access to savings and borrowing for the dowry, a payment that is required at marriage, but also face a borrowing constraint. The main predictions regarding marriage decisions are that positive income shocks enable the household to delay marriage, and negative income shocks induce the household to marry early, as long as the dowry payment does not exceed the borrowing limit.

By using an instrumental variable (IV) estimation approach, I can address the endogeneity that would arise in estimating the direct effect of Child Marriage on children's health outcomes, since there are omitted variables that are likely to be correlated with both Child Marriage and children's health outcomes. For example, consider the status of women in Bangladesh. Socio-cultural norms dictate the subservience of women-to-male relations (Joseph and Nağmābādī 2003). This can entail a lack of power and independence in various aspects of their life. Coupled with unequal access to education, employment and health information (Stromquist 2014), these characteristics can have a significant influence on marriage age decisions and the health outcomes of their children.

The results from the IV estimation indicate that the health outcomes of children are adversely affected by Child Marriage. I find that Child Marriage increases the likelihood of stunting in children by 8.33 percentage points. I conduct additional analyses using 
different measures of rainfall shocks, and find similar and significant increases in stunting from children born to mothers in Child Marriage unions. I also examine the extent to which Child Marriage affects stunting and find that children are more likely to be severely stunted. The effect of Child Marriage on moderate stunting yields results that are not significantly different from zero, indicating that the predominant effect on children's health outcomes is concentrated through increases in severe stunting.

This paper distinguishes itself from existing research by establishing causality between Child Marriage and children's outcomes. While there is a body of research that examines the causal effect of Child Marriage on women's educational outcomes (Field and Ambrus 2008, Nguyen and Wood 2012) and explores the association between Child Marriage and the health consequences for the child bride and their children (Nour 2009, Nour 2006, Raj et al. 2010, Le Strat, Dubertret and Le Foll 2011, Ahmed et al. 2013), none has directly generated estimates of the potential impact on children produced from these unions. These findings justify the need for considerations related to expanding and providing resources to children from Child Marriage unions, as opposed to present efforts aimed solely at the welfare of the child bride.

The rest of the paper is divided as follows. Section 1.2 discusses the background of Child Marriage in Bangladesh and reviews related economic literature. In Section 1.3, I present a simple conceptual framework to motivate the identification strategy, and in Section 1.4, I describe the datasets used. Section 1.5 details the empirical strategy. Section 1.6 reports the main empirical results and Section 1.7 presents robustness checks. Finally, Section 1.8 concludes. 


\subsection{Child Marriage}

\subsubsection{Background}

Marriage is a universal construct in Bangladeshi society. It is a necessity for females as they are only afforded respect and status through male relationships (Chen 1983). There are social-related costs of having unmarried mature daughters in the household; the family may face exclusion from their village and risk being socially ostracized for not following the norm. Furthermore, marriage is considered as a sign of success for the family and a means to elevate their respect and prestige within their village (Myers 2013).

The International Centre for Research on Women's Demographic Health Survey, for the period 2002-2011, ranks Bangladesh as the country with the fourth highest rate of Child Marriage in the world (Verma et al. 2013). The most current estimates show that 52 percent of females are married before the age of 18 , and 18 percent are married before the age of 15 (UNICEF 2016). In accordance with the 1929 Child Marriage Restraint $\mathrm{Act}^{2}$, the minimum age for a woman to marry is 18 . The punishment for Child Marriage, as set forth in sections 4 through 6 of the Child Marriage Restraint Act, is imprisonment of up to one month, or a fine of one thousand Taka, or both. Child Marriage remains prevalent in Bangladesh for numerous and interrelated reasons. Firstly, the national law is routinely ignored and enforcement does not occur very often. Further complicating matters are religious personal laws, which can permit marriage at ages younger than 18 in some

\footnotetext{
2 The Child Marriage Restraint Act No. XIX of 1929
} 
circumstances (Hossain 2013). For example, under Muslim personal law, marriage of a female is allowed at 14 years of age.

Second, the economic circumstances of the bride's household, coupled with the discriminatory attitude towards girls can fuel Child Marriage. Generally, parents have lower aspirations for daughters than sons (Beamen et al. 2012). While sons are seen as the future wage earners, daughters are considered to be an economic burden (White 1992). For households that are poverty-stricken, Child Marriage is used as a coping strategy to lessen their costs. Girls in Bangladesh have reported a lack of food and drought occurrence as causes for being married off (Myers 2013). The marriage provides an avenue for the bride's household to transfer the burden to the groom's family.

Third, the practice of dowry which increases with the age of the girl at marriage (Field and Ambrus 2008, Mathur et al. 2003) places additional pressure on parents to marry daughters early. Marriage payments in the form of dowry originated amongst wealthy families in Bangladesh in the 1950s and then spread to rural areas in the 1960s before becoming a norm amongst poorer families in the early 1970s (Amin and Cain 1997, Lindenbaum 1981, Ambrus, Field and Torero 2008). The payment of dowry has taken on many different forms. Lindenbaum (1981) found that the list of groom demands included jewelry (watches, rings), household objects (radios, television sets, record players) and forms of transportation (car, bicycle or motorbike) in Bangladesh and India. Cash payments have been the most common form of dowry transactions in recent years (Amin and Cain 1997). Even for households facing a poor financial situation, the dowry payment does not deter them from Child Marriage. The one-time dowry payment is weighed against the burden of providing ongoing support for the daughter, and is seen as the preferred option 
(Myers 2013). The dowry is raised by asset stripping at the time of the wedding; the bride's parents may sell livestock, agricultural products and land to raise money for the dowry payment. In other cases, they borrow money from the local moneylender or utilize microcredit ${ }^{3}$ (Chowdhury 2010, Huda 2006).

Finally, the preferences of the groom's household also drive the practice of Child Marriage. A groom's household seeks out a young girl as a bride to ensure sexual purity and to maximize fertility. The ultimate goal of the marriage is to build a family and designate an inheritor for the future of the family. Additionally, child brides are easier to dominate and more likely to be submissive to the demands of the groom and his family (Chowdhury 2004). In comparison, girls over the age of 18 are more independent and selfaware. Education is another element; older girls are less likely to tolerate mistreatment and more able to develop a bond with the groom, a factor that could potentially destabilize his family unit (Ross 2009).

\subsubsection{Consequences of Child Marriage}

Child Marriage is associated with a range of adverse physical and mental health consequences for the child bride. The lack of access to information on reproductive health, coupled with the inability to make decisions within the household contributes to child brides experiencing a higher probability of unwanted pregnancies (Union 2015). Early pregnancy presents a greater risk for maternal morbidity and mortality, as child brides are physically and emotionally unprepared for birth (Raj et al. 2009, Malhotra 2010, Mayor

\footnotetext{
${ }^{3}$ Given the illegal nature of dowry according to the Dowry Prohibition Act of 1980, other assets (cows, rickshaw vans) are shown to the microcredit officer as proof of the loan use (Geirbo and Imam 2006).
} 
2004). To illustrate, child brides are vulnerable to obstructed labor, a condition caused by the girl's pelvic bones being too small to deliver. In comparison to adult women giving birth, they are more likely to have pregnancy and delivery-related complications. These can include the development of hypertension, eclampsia, postpartum hemorrhage and fistulas (Nour 2006). These considerable risks can translate into harmful conditions for their infants, through a higher probability of low birth weight and infant morbidity and mortality (Nour 2009).

While Child Marriage exposes girls to numerous harmful physical health conditions, their mental health is also affected. Child brides suffer from social isolation; they are often confined to the groom's household and prevented from visiting former family and friends (Nour 2009, Le Stat et al. 2011, Parsons et al. 2015). Child brides are also usually forced to end their education early and are unable to access schooling development programs (Vogelstein 2013). The removal from school does not just represent the loss of educational opportunities but, moreover, the ability to interact with peers of the same age and socialize outside of the family unit (UNICEF 2014). This undermines the development of the child bride as it obstructs the formation of an independent identity. These factors can contribute to an increased risk of depression, anxiety and suicide (Carbone-Lopez et al. 2006). The high susceptibility to domestic violence furthermore contributes to these adverse outcomes. Rahman et al. (2014) find that, in comparison to later-married women, child brides in Bangladesh are more likely to experience physical abuse. Among the South Asian countries of Bangladesh, Pakistan, India and Nepal, the prevalence of physical partner violence is highest in Bangladesh at 40 percent (UNICEF 2014). Child brides often face physical abuse 
for their failure to fulfill the sexual demands of their husbands and expectations of their new family unit (Myers 2013).

The physical and mental health consequences of Child Marriage highlighted here indicate that Child Marriage compromises the development and well-being of the child bride. A priori, this in turn should adversely impact the outcomes of their children. Yet, there is an information deficiency on the scale of damage caused by Child Marriage on children's outcomes. The only literature to address this, to the best of my knowledge, is from Raj et al. 2010. Raj et al. (2010) evaluate associations between Child Marriage and morbidity and mortality of children under the age of 5 in India. Although their findings document children born to mothers from Child Marriage unions are more vulnerable to malnutrition than other outcome measures, their analyses are cross-sectional and causality cannot be assumed. Their results inform my research on the most suitable measure of health outcomes for children.

\subsubsection{Related Literature}

The existing economic literature on Child Marriage extends into two different directions. The first is involved in estimating the causal effect of Child Marriage on the child bride's outcomes. Field and Ambrus (2008) generate possibly the first estimates of the causal effect of Child Marriage on education outcomes. Using the age of menarche as an instrument for marriage age, they find that for girls in Bangladesh, a 1-year postponement of marriage between the ages of 11 and 16 increases schooling and adult literacy. Nguyen and Wodon (2012) find similar evidence for child brides in Africa, using the contemporaneous and past incidence of Child Marriage at the level of the primary sampling 
unit in which the girls reside as their instrument. Findings from Lloyd and Mensch (2008) using data on the drop-out rates for girls in Sub-Saharan Africa, also indicate Child Marriage affects education outcomes.

The second is based on understanding the driving forces behind the practice, specifically the relationship between marriage payments and the incidence of Child Marriage. Corno and Voena (2016) provide causal evidence of the association between Child Marriage and adverse income shocks that affect the potential bride's household in a setting that engages in bride price payments. Using rainfall shocks as a proxy for income shocks and data from Tanzania, their findings show that adverse income shocks increase the probability of Child Marriage. Corno, Hildebrandt and Voena (2016) also examine the impact of income shocks on Child Marriage. Their analysis differs by setting and in the construction of rainfall shocks, in which a measure of drought and flood is used. They show that drought has opposite effects on the hazard of Child Marriage in Africa and India and that flood has no impact in both. Their explanation for the difference in effects of a drought relies on the differences in marriage payments in Africa (bride price) and India (dowry). Their methodology influences my research, through the insight gained from the relationship between rainfall shocks and the probability of Child Marriage.

\subsection{Conceptual Framework}

I present a simple model that intends to capture the relationship between idiosyncratic income shocks and parental marriage decisions within a given period. In the basic framework, a household consists of parents and a daughter, where parents make all decisions and have an obligation towards marrying their daughter. 
At the time of the daughter's marriage, parents are required to make a one-time dowry payment. They have access to savings and borrowing for the dowry payment. In cases of borrowing, parents face a borrowing constraint. The dowry payment increases with the age of the daughter at marriage. I assume the minimum age of marriage to be 12 years as girls are withheld from the marriage market until the onset of puberty (Field and Ambrus 2008) and the average age for menstruation in Bangladesh is 12 years.

The household exists for two periods (present and future) and is exposed to idiosyncratic shocks in the first period (present) during which the daughter is between 12 and 17 years of age. The second period (future) occurs through the time that the daughter is 18 years and older.

Parents are altruistic and derive utility from delaying the daughter's marriage due to the negative consequences that can be imposed from marriage before the age of 18 . However, in times of economic hardship, parents may consider marriage as a strategy to lessen financial burden on the household.

The model presents important predictions regarding the timing of marriage - first, a positive income shock increases the likelihood of marriage in the second period. Second, a negative income shock increases the likelihood of marriage in the first period. However, if the dowry payment exceeds the borrowing limit, marriage is delayed to the second period.

\section{Setup}

Parents live for 2 periods of time: period 1(present) and period 2(future). They receive utility $u\left(c_{t}\right)$ over consumption in each period, and from the well-being of their daughter through an altruism parameter $\alpha \epsilon(0,1)$. 
In the first period, the income shock occurs. Within the first period, parents decide how much to consume $\left(c_{1}\right)$ and whether or not to marry their daughter $\left(m_{1} \epsilon\{0,1\}\right)$, where $m_{1}=1$ if marriage occurs in the first period. If the marriage takes place, they must pay the dowry $(D)$. In the second period, parents decide how much to consume $\left(c_{2}\right)$ and pay a dowry $D(1+d)$ if the daughter is married at that time, $d>0$.

Parents can borrow in period $1\left(b_{1}>0\right)$, but they face a borrowing constraint $(\bar{b})$. The amount borrowed in the first period has to be repaid at the real interest rate $(r)$ in the second period. Similarly, parents can save in the first period $\left(b_{1}<0\right)$ and earn the real interest rate $(r)$ on their savings in the second period. They derive utility $\alpha\left(1-m_{1}\right)$ from the timing of their daughter's marriage.

\section{Marriage Decision}

Parents possess the following lifetime utility function:

$V\left(m_{1}\right)=\log c_{1}+\frac{1}{\rho} \log c_{2}+\alpha\left(1-m_{1}\right)$

And they face the following budget constraints:

$$
\begin{aligned}
& c_{1}+D m_{1}=y_{1}+b_{1} \\
& b_{1}(1+r)+c_{2}+D(1+d)\left(1-m_{1}\right)=y_{2}
\end{aligned}
$$

I make the following assumptions. For simplicity, in the absence of income shocks, households receive the same income in both periods, $y_{1}=y_{2}$. Households face a borrowing constraint, $b_{1}<\bar{b}$. And lastly, the rate of interest is less than the increase in cost of dowry, $<d$. if $r>d$, households will always choose marriage in the second period. 
The budget restrictions if the daughter is married in the first period, thus $m_{1}=1$ are:

$$
\begin{aligned}
& c_{1}+D=y_{1}+b_{1} \\
& b_{1}(1+r)+c_{2}=y_{2}
\end{aligned}
$$

This can be consolidated to yield the lifetime budget constraint:

$c_{1}+D+\frac{c_{2}}{1+r}=\frac{y_{2}}{1+r}+y_{1}$

After maximizing the utility function (1.1) subject to the lifetime budget constraint (1.3a), the levels of consumption obtained by marrying their daughter in the first period are:

$$
\begin{aligned}
& c_{1}=\frac{\rho}{1+\rho}\left[\frac{y_{2}}{1+r}+y_{1}-D\right] \\
& c_{2}=\frac{1+r}{1+\rho}\left[\frac{y_{2}}{1+r}+y_{1}-D\right]
\end{aligned}
$$

Hence, the value of marrying their daughter in the first period is equal to:

$$
V\left(m_{1}=1\right)=\log \left[\frac{\rho}{1+\rho}\left[\frac{y_{2}}{1+r}+y_{1}-D m_{1}\right]\right]+\frac{1}{\rho} \log \left[\frac{1+r}{1+\rho}\left[\frac{y_{2}}{1+r}+y_{1}-D m_{1}\right]\right]
$$

If the daughter marries in the second period, parents will save in the first period and use the savings plus interest earned in the second period towards consumption and the dowry payment. The budget identities are given by:

$$
\begin{aligned}
& c_{1}=y_{1}+b_{1} \\
& b_{1}(1+r)+c_{2}+D(1+d)=y_{2}
\end{aligned}
$$

This can be combined into the following lifetime constraint:

$$
c_{1}+\frac{D(1+d)}{1+r}+\frac{c_{2}}{1+r}=\frac{y_{2}}{1+r}+y_{1}
$$


After maximizing the utility function (1.1) subject to the lifetime budget constraint (1.3b), the level of consumption obtained by marrying their daughter in the second period is:

$$
\begin{aligned}
& c_{1}=\frac{\rho}{1+\rho}\left[\frac{y_{2}}{1+r}+y_{1}-\frac{D(1+d)}{1+r}\right] \\
& c_{2}=\frac{1+r}{1+\rho}\left[\frac{y_{2}}{1+r}+y_{1}-\frac{D(1+d)}{1+r}\right]
\end{aligned}
$$

The value of marrying their daughter in the second period is equal to:

$$
V\left(m_{1}=0\right)=\log \left[\frac{\rho}{1+\rho}\left[\frac{y_{2}}{1+r}+y_{1}-\frac{D(1+d)}{1+r}\right]\right]+\frac{1}{\rho} \log \left[\frac{1+r}{1+\rho}\left[\frac{y_{2}}{1+r}+y_{1}-\frac{D(1+d)}{1+r}\right]\right]+\alpha
$$

The dowry payment increases with age of the daughter at marriage. For example, parents of a 12-year-old girl will pay a smaller dowry than the parents of a 16-year-old. Coupled with the borrowing constraint that parents face, two cases arise-first, the constraint is not binding and the dowry is less than or equal to the borrowing limit and second, the constraint is binding and the dowry exceeds the limit on borrowing. We assume that absent a negative income shock in the first period, the borrowing constraint is not binding. Given that parents have to marry their daughter eventually and if $y_{1}=y_{2}$, if they cannot marry her in the first period they would not be able to marry her in the second period when the amount of the dowry they need to pay is higher, $D(1+d)$.

We allow for income shocks to occur only in the first period, as this is the period in which parents can make any meaningful decision. Parents face a choice of whether to marry their daughter in the first period or the second period. The parents' decision to marry the daughter in period $2\left(m_{1}=0\right)$ is based on whether the value of marrying in period 2 is greater than the value of marrying in period 1:

$$
V\left(m_{1}=0\right)-V\left(m_{1}=1\right)>0
$$


If the borrowing constraint is not binding, we can derive the relationship between the likelihood of marrying in the second period and change in income in the first period from $(1.4 \mathrm{~b}) \geq(1.4 \mathrm{a})$. $\frac{\partial\left(V\left(m_{1}=0\right)-V\left(m_{1}=1\right)\right)}{\partial y_{1}}=\left(\left(\frac{y_{2}}{1+r}+y_{1}-\frac{D(1+d)}{1+r}\right)^{-1}-\left(\frac{y_{2}}{1+r}+y_{1}-D\right)^{-1}\right)\left(1+\frac{1}{\rho}\right)>0$

Thus, in the event of a positive income shock ( $y_{1}$ increases), the likelihood of marriage being delayed increases. In the event of a negative income shock if the borrowing constraint is not binding, a fall in $y_{1}$ increases the probability that the daughter is married in period 1 . However, if the borrowing constraint is binding, such that $\left(D>y_{1}+\bar{b}\right)$, parents no longer have a choice between marrying in the first period or second period; they are forced to delay the marriage of their daughter until the second period. There may be concerns about whether or not parents will be able to afford the dowry payment in the second period. The older age of the girls in the second period may facilitate more employment opportunities ${ }^{4}$.

\subsection{Data}

\subsubsection{Health Outcomes and Marriage Data}

The primary source of data for the analysis comes from the 1999-2000 Bangladesh Demographic and Health Survey (BDHS). The survey was authorized by the National Institute of Population Research and Training (NIPORT) and implemented by a

\footnotetext{
${ }^{4}$ Anecdotal evidence from Chowdhury (2010) shows that the girls that are employed are expected to contribute their earnings to savings for the dowry payment. This has the potential to boost savings in the second period, putting parents in a better position to afford the dowry at marriage.
} 
Bangladesh research firm, Mitra and Associates. The survey comprises of household and individual-level information on 9854 households in 341 primary sampling units (referred to as clusters). The survey interviewed 10544 married women between the ages of 10 and 49 , and contained a record for every child of an interviewed woman that was born in the 5 years prior to when the survey was conducted.

The BDHS dataset proves to be suitable for investigating the effect of Child Marriage on children's health outcomes for several reasons. Firstly, it allows for the matching of each child's record to data from their respective mother. Of particular importance is the age of the mother at marriage. Child Marriage, one of the main variables of the analysis, is constructed using this information. The age at marriage of the mother distinguishes clearly whether the child was born from a Child Marriage union or a non-Child Marriage union.

Secondly, from the BDHS dataset there is a detailed provision of information relating to characteristics of the child's health, including anthropometric measurements, immunization coverage, vitamin A supplementation, breastfeeding practices, and the prevalence and treatment of childhood diseases. The other main variable of the analysis, health outcomes, is measured from one of the anthropometric indicators. The height-forage index is used over other measures of nutritional status (weight-for-height index and weight-for-age index) because of its advantage of being robust to transitory spells of disease and seasonality in diet. The weight-for-age index, which indicates whether a child is underweight, is a misleading indicator of health outcomes due to the absence of the height measure. For instance, a tall child with adequate weight for his stature will be classified as underweight. The weight-for-height index is also a poor proxy for health 
outcomes because it can be influenced heavily by economic and environmental factors. The index measures the condition of wasting, which can be reversed, depending on the availability of food (Ray 2013, Gorstein et al. 1994, Dewey et al. 2005). The height-forage index proves to be the best measure of health outcomes, as it measures stunting, a condition reflecting the cumulative effect of malnutrition (Deshmukh, Sinha and Dongre 2013, Black et al. 2013). The child is considered stunted if the height-for-age z-score is below minus two standard deviations from the median of the National Center for Health Statistics/World Health Organization (NCHS/WHO) reference population (WHO 1995, Prendergast and Humphrey 2014). This index is a standard measure of children's health outcomes as shown by Edlund and Rahman (2005), Dulfo (2000) and Atkin (2009). The World Health Organization advises limiting analysis of height measures to children of a young age, specifically between 0 to 5 years (WHO Working Group 1986). This restriction is adhered to, as the analysis includes only children in this specific age range.

Finally, the BDHS helps to implement the identification strategy by providing geographic information on each cluster. The latitude and longitude of the center of each cluster is needed for matching rainfall shocks to the right weather grid cell.

The final sample consists of 1555 children between the ages of 0 to 5 years old that have non-missing information on health outcomes. I restrict the sample to first-born children's cases to minimize bias. The sample only contains data on children born in the last 5 years preceding the survey (from 1994 to 2000). The inclusion of all children leads to a selection issue as households with many children are more likely to be included. Households with many children will systematically differ from other households. Additionally, data are only available for the last child and or the second-to-last child. 
Information such as birth order and birth spacing is missing for all children born before the year of 1994. These factors, when excluded, will confound the analysis and lead to erroneous results.

Figure 1.1 displays the distribution of the age at marriage for the mothers of the children in the sample. The highest frequency of marriages is recorded at age 13, followed by ages 14 and 15. In Table 1.1, summary statistics for the sample are reported. On average, the age at marriage is 15.81 . Approximately 76 percent of children are born to mothers from a Child Marriage union and 42 percent of children are born to mothers who were married before the age of 15 . Birth characteristics display that 45 percent of births occur before the age of 18 and the mean age of the mother at birth is 18.75 years. Approximately half of the children (48 percent) are reported as stunted. When examining the different levels of stunting, 29 percent are reported as moderately stunted and 19 percent are severely stunted. Demographic characteristics show that the majority of the sample is from rural areas, with only 29 percent living in urban areas. A large majority of the parents are educated; 72 percent of mothers and 64 percent of fathers have a primary education. However, the literacy level for mothers is lower at 50 percent. Regarding the levels of wealth of the household, approximately 45 percent of the children are from households above the middle quintile (fourth and highest quintile) of wealth and 36 percent are from households below the middle quintile (lowest and second quintile).

\subsubsection{Rainfall Data}

A main assumption in the analysis is that the timing of marriage is influenced by the variation in the household's income. Fluctuations in rainfall are a major contributing factor 
to income variation in Bangladesh, due largely to the household's dependence on the agricultural industry. The most recent estimates show that the agricultural sector employs 47.3 percent of the labor force and contributes 16.33 percent of the GDP (Bank 2014). In the time period preceding the survey, the agricultural sector was even more central to the economy, employing approximately 64 percent of the labor force and accounting for 30 percent of the GDP. Rice has consistently remained the principal agricultural crop, occupying 80 percent of agricultural land use and contributing to 90 percent of total grain production (Asaduzzaman et al., 2012).

I obtain rainfall data from the University of Delaware dataset (Matsuura and Willmott 2007). This dataset provides monthly total precipitation spanning from 1900 to 2010 interpolated to a 0.5 by 0.5 degree grid resolution. For every year between 1960 and 2010 , I use the Global Positioning System(GPS) information from the BDHS dataset to match each of the 341 clusters to its weather grid cell in which it is stationed.

Rainfall variation is measured in numerous ways in the literature (Miguel, Satyanath and Sergenti 2004, Burke, Gong and Jones 2014, Corno and Voena 2015, Corno, Hildebrandt and Voena 2016). In line with Burke, Gong and Jones (2014), I use a binary indicator for rainfall shocks because continuous measures are potentially problematic if the high years of rainfall were to offset the low years.

To construct a measure of drought and flood from the rainfall data, I sum the total precipitation for each year from 1960 to 2010 and compute the historical mean and standard deviation of each cluster. Using this information, a normalized index is created. I define a drought shock as a rainfall realization that is less than $1.5 \mathrm{SD}$ below the cluster mean. Symmetrically, a flood shock is defined as a rainfall realization that is $1.5 \mathrm{SD}$ above the 
cluster mean. Using this particular cutoff level to define a shock is informed by previous literature, in relation to the annual precipitation anomalies during a drought (Burke, Gong and Jones 2014, Cayan et al 2010, Flato and Kotsadam 2014).

Drought and flood shocks are constructed for mothers when they were between the ages of 12 and 17. The minimum age is assumed to be 12, as girls are withheld from the marriage market until the onset of puberty (Field and Ambrus 2008) and the average age for girls to start menstruating in Bangladesh is 12 years. Ages after 17 are excluded, since the issue at hand is Child Marriage.

Using this specific construction of rainfall shocks presents a significant advantage to the empirical analysis. Given that shocks are defined relative to the historical mean in each particular cluster and the cut-off used in each cluster location is the same, all clusters have equal probability of experiencing a shock in any particular year. This random variation in rainfall shocks is exploited to address endogeneity concerns.

\subsubsection{Rainfall Shocks and Agricultural Output}

Drought shocks are unquestionably adverse income shocks because of their negative impact on agricultural output (Cavallo et al. 2013, Fomby et al. 2013, Kazianga and Udry 2006). In contrast, there is no consensus on the effect of flood shocks. The majority of the literature that considers flood shocks as adverse in their analysis either examines the impact of the most severe floods or uses exposure to an extreme flood event. For example, Rosales-Rueda (2014) exploits extreme floods during the El Nino phenomenon; Ghimire and Ferreira (2012) assume floods are negative shocks to GDP growth using data on large flood events; Fair et al. (2013) use evidence from the 2010-11 Pakistani flood, which was 
deemed as the worst flood in Pakistan's history; and Rana and Islam (2015) explore the relationship between agricultural production and floods in Bangladesh, focusing on exposure to river floods, which are deemed the most severe floods. The literature that finds a positive impact on floods takes into consideration the differences in the intensity of flood shocks. For instance, Fomby et al. (2013) finds a difference in the effects of moderate and severe floods - only moderate floods tend to have a positive effect by raising land productivity. Similarly, Cunado and Ferreira (2014) account for flood magnitude by constructing four different types of flood shocks. They also find a positive impact from a flood shock in developing countries that rely more on traditional agricultural methods. Floodwaters can enrich the soil and make agricultural lands more fertile by carrying and depositing nutrients and sediments over plains (Brebbia 2013). For rice paddies, this is extremely beneficial as it removes the need for artificial fertilizer and provides fresh water for irrigation use.

To further explore the relationship, I use the measures of drought and flood to estimate the effect on the rice crop in Bangladesh. I source yield data from the Food and Agriculture Organization Corporate Statistical Database (FAOSTAT). Due to the provision of yield data available only on a country level basis and not by GPS or cluster identification, I construct a country-level measure of rainfall shocks from 1960 to 2010. Using the same cut-off level, I define a drought (flood) shock as 1.5 standard deviations below (above) the mean. I repeat this empirical analysis to explore the effect of rainfall shocks on wheat and total cereals. Wheat is the second most important grain in Bangladesh and total cereals include all the grains: rice, wheat, millet, barley and sorghum. 
Table 1.2 shows the results of the rainfall shocks on the crop yields. A drought-related rainfall shock has a negative effect on rice, wheat and cereal yields while a flood shock has a positive effect on all of the crop yields. Although the results are not statistically significant, which may be due to the limited number of observations, the regression results highlight that there is a differing effect of a drought and flood shock on crop yields. This contradicts the expectation that a flood shock should be treated as an adverse income shock for Bangladeshi households.

The resulting estimates from the regressions of country level rice yields and wheat yields on rainfall percentile are shown in Figures 1.2 and 1.3 respectively. From these two figures, lower rainfall realizations lead to lower rice and wheat yields. Extremely high rainfall realizations (after the $80^{\text {th }}$ percentile) have different effects, leading to greater rice yields but causing wheat yields to start declining.

Using information from the analysis and past literature, I conclude that the drought shocks serve as negative income shocks to households. The effect of flood shocks is dependent on the severity and magnitude. Given the particular definition of a flood shock in my analysis, the results from the regressions on crop yields and the past literature on the positive effects of moderate flood shocks, I conclude that flood shocks should be considered as a positive income shock to Bangladeshi households. 


\subsection{Empirical Strategy}

To investigate the effect of Child Marriage on children's health outcomes, I estimate the following:

$Y_{i, c}=\alpha_{0}+\alpha_{1} C M_{i, c}+\alpha_{2} X_{i, c}+\gamma_{t}+\delta_{c}+\varepsilon_{i, t, c}$

where $Y_{i, c}$ is the health outcome indicator for stunting of child $i$ in cluster $c$, it takes the value of 1 if the child's height-for-age z-score is less than 2SD from the reference median and 0 otherwise. $C M_{i, c}$ is a cardinal variable that takes the value of 0 if the child's mother was married at 18 years and above, and 1 if married at age 17,2 if married at age 16,3 if married at age 15 and so forth. $X_{i, c}$ is a vector representing the following controls: parental education, wealth, urban location, teenage (adolescent) pregnancy and flood shock at birth ${ }^{5}$. Parental education controls include whether the mother is literate, the mother has a primary education and the father has a primary education. Wealth levels are denoted by four quintiles: lowest, second, fourth and highest. The lowest quintile represents the poorest 20 percent of households and the highest quintile represents the richest 20 percent of households in Bangladesh.

Estimation of the model in Equation (1.5), using Ordinary Least Squares (OLS), fails to uncover the causal relationship between Child Marriage and children's health outcomes due to the presence of endogeneity. There are unobservable variables that can induce a correlation between Child Marriage and the error term and lead to an asymptotic bias. To illustrate the endogeneity problem, consider the disadvantageous status of women in Bangladesh. Socio-cultural norms dictate that women must be subservient to all male

\footnotetext{
${ }^{5}$ The need for the control flood shock at birth, will be explained further after discussion of the instrumental variable approach.
} 
relations. Further, women tend to be secluded with restricted access to education, employment and health information (Stromquist 2014). Overall, this can contribute to a loss of independence and power in various aspects of their life. These factors, in turn, are liable to influence marriage age decisions and the health outcomes of their children.

To address the endogeneity problem in estimating the causal effect of Child Marriage on children's health outcomes, I employ a two-step methodology where drought and flood shocks are used as instruments for Child Marriage. Rainfall shocks are an important source of income variation, due largely to the household's dependence on the agricultural sector. Rainfall-related shocks generate significant changes in the household's decision to enter into a Child Marriage union. Earlier studies have utilized rainfall shocks as instruments for the age at marriage; Corno and Voena (2015) find that adverse rainfall shocks increase the probability of marrying by age 18 in Tanzania and Corno, Hildebrandt and Voena (2016) show that drought shocks have differing effects-increasing the early marriage hazard in Africa where the brides' families receive bride prices, and decreasing it in India, where the brides' families pay dowries.

The two-stage model involves estimating:

$$
\begin{aligned}
Y_{i, c}= & \alpha_{0}+\alpha_{1} C M_{i, c}+\alpha_{2} X_{i, c}+\gamma_{t}+\delta_{c}+\varepsilon_{i, t, c} \\
C M_{i, c}= & \beta_{0}+\beta_{1} \text { DroughtShock At Age } a_{i, c}+\beta_{2} \text { Floodshock At Age } a_{i, c}+\beta_{3} X_{i, c}+ \\
& \gamma_{t}+\delta_{c}+\vartheta_{i, t, c}
\end{aligned}
$$

where DroughtShock At Age $a_{i, c}$ and Floodshock At Age $a_{i, c}$ are the instruments. They are both binary indicators that take the value of 1 if the mother of child $i$ experienced a drought (flood) shock at age $a$ in cluster $c$ and 0 otherwise. I use ages ( $a$ ) from 12 to 17 in the regression analysis. The minimum age is assumed to be 12, as girls are withheld 
from the marriage market until the onset of puberty (Field and Ambrus 2008) and the average age for girls to start menstruating in Bangladesh is 12 years. Drought (flood) shocks are defined as 1.5 standard deviations below (above) the historical mean in each cluster. Using this specific construction of shocks means that all clusters have equal probability of experiencing a shock in any particular year. By exploiting the random variation in rainfall shocks, I can correct for any endogeneity bias.

$X_{i, c}$ is a vector representing the controls described earlier. The control, flood shock at birth, is the average effect of flood shocks one year before, the year of, and one year after, child birth. For children born to mothers who are still minors, these cases introduce a natural correlation between a shock in the birth year of the child and when the mother is between 12 and 17 years of age. This shock becomes part of the error term leading to instruments having a correlation with the error term. To address this problem, the control takes into account the average effect of a flood shock in the conception stage (year before child birth), the year of birth, and the lactation stage (year after child birth). Cluster fixed effects $\left(\delta_{c}\right)$ and year of birth fixed effects $\left(\gamma_{t}\right)$ are included to account for any unobserved differences in the cluster and in the year the child was born.

\subsection{Results}

\subsubsection{OLS Results}

Results from Ordinary Least Squares (OLS) estimates for stunting are reported in Table 1.3. Columns 1 to 3 report results without and with year of birth fixed effects. The results 
indicate that women who are married before the age of 18 worsen their children's health outcomes by increasing the likelihood of stunting by 1.73 percentage points (column 2).

In column 3, the model specification is expanded to include a control for whether the mother was a teenager at the time of birth. The Child Marriage estimate in both model specifications (columns 2 and 3) is about the same distance from zero, but it is less statistically significant in column 3. By introducing the additional control, the high correlation between Child Marriage and teenage pregnancy at 0.662 (Table A1) induces the problem of multicollinearity. This causes the standard error to increase, thereby lowering the significance level for the Child Marriage estimate. However, the overall fit of the model is not affected; the R-squared value is relatively the same, with a change of 0.0002 .

In comparison to the instrumental variable (IV) estimates reported in Table 1.5, OLS underestimates the results of the effect of Child Marriage. This implies that the OLS estimate is attenuated due to the presence of the omitted variable bias.

\subsubsection{Results}

\subsubsection{First-stage Regression Results}

For identification of an IV model using drought and flood shocks as instruments, there has to be a strong correlation between these weather shocks and the probability of Child Marriage. First-stage regression results with and without fixed effects are reported in Table 1.4. The coefficients are slightly larger in magnitude when year fixed effects are included 
but are not statistically different from the baseline specifications. The differences in the F statistic and R-squared are relatively minor.

The results in the first three columns estimate the average effect of drought shocks and flood shocks across ages 12 to 17 . As expected from the conceptual framework, there is a negative and statistically significant relationship between a flood shock and the probability of marrying before 18 . Women that experience a flood shock between the ages 12 and 17 (column 3) have a 3.29 points lower probability of being in a Child Marriage union. This effect is statistically significant at the 1 percent level with an F statistic of 132.2. The Rsquared value indicates that 50.8 percent of the total variance is being explained by the variables used. In contrast, the sign on the coefficient of the drought shock (column 3) appears to be inconsistent with the predictions from the conceptual framework. However, its validity remains highly questionable, given that the coefficient is small in magnitude and not significantly different from zero.

To examine the distinct effects of weather shocks at different ages, dummy indicators of a drought shock and flood shock between the ages 12 and 13, 14 and 15, 16 and 17 are presented in columns 4 to 6 . The greatest effects of a flood shock on marrying before 18 occur in the age category of 12 to 13 and 16 to 17 (column 6). The age category 14 to 15 is not statistically significant but maintains, as expected, a negative relationship. A one standard deviation increase in a flood shock at ages 12 to 13 leads to an 8.8 percentage point decreased probability of entering into a Child Marriage and a one standard deviation increase in a flood shock between 16 and 17 leads to an 11.2 percentage point lower probability of marrying before 18 . These effects are statistically significant at the 1 percent level, with an F-statistic of 101. There is no significant difference in the R-squared value 
(column 3 and 6) between rainfall shocks at specific age categories and averages of rainfall shocks on the probability of Child Marriage.

Drought shocks experienced between the ages of 12 and 13, and 14 and 15 (column 6), are not statistically significant but retain the positive effect on Child Marriage, as predicted from the conceptual framework. In the age category 16 to 17, the coefficient for drought shocks is negative and statistically significant from zero. This change from a positive effect in the younger age categories to a negative effect in the older age category is in line with the predictions from the framework. Girls at 16 to 17 years will have to pay a higher dowry at marriage in comparison to girls in the younger age categories. For a household hit by a drought shock, the higher dowry may lessen their ability to marry the daughter at the time of the shock. Additionally, employment opportunities that are only available to the daughter at later ages may be a factor contributing to the decline in Child Marriage in the age category 16 to $17^{6}$.

\subsubsection{Child Marriage and Health Outcomes}

Results from IV specifications are presented in Table 1.5, with and without year fixed effects. The first three columns present results using the average effects of drought shocks and flood shocks across ages 12 to 17 as instruments. The specification in column 2 shows that Child Marriage unions lead to a 5.16 percentage point increase in the likelihood of stunting in children. The effect is statistically significant at the 1 percent level and is robust

\footnotetext{
${ }^{6}$ In Bangladesh, the rise of the garment industry in the 1980s and its facilitation of child labor has made more employment opportunities accessible to girls. Women's labor force participation rate rose continuously from 1991 to 2010, with the highest increase reported among the younger age groups (Rahman and Islam 2013, Wiebecke and Raham 1996).).
} 
to the inclusion of controls for urban location, parental education, wealth levels and flood shocks at the time of child birth. The coefficients on the father's education and the higher wealth levels of the household (fourth quintile and highest quintile) are negatively correlated with stunting. This is not surprising as low caregiver education and poor home environment (inadequate sanitation and water supply) are contributing factors of stunting.

The coefficient on flood shock at birth is negatively correlated with stunting. This confirms that a flood shock represents a positive flow of income into the household. One of the major causes of stunting is inadequate nutrition, which is fueled by food insecurity, infrequent feeding, poor sanitation and water supply (Onis et al. 2013, WHO 2013, Dewey and Begum 2011). If flood was considered as a negative income shock to the household, then food availability and characteristics of the home environment are likely to worsen, in turn increasing the likelihood of stunting. Thus, the negative relationship between the flood shock at birth and stunting corresponds to a flood shock being a positive income shock to the household. In column 3, the control for teenage pregnancy is added to the model specification. The high pairwise correlation $^{7}$ between Child Marriage and teenage pregnancy causes the standard error to rise. In comparison to the OLS estimation, the increase of the standard error in the IV estimation is more pronounced. Consequently, there is a decrease in the statistical power of the Child Marriage coefficient. The results indicate that Child Marriage unions increase stunting by 8.33 percentage points and are statistically significant at the 10 percent level.

\footnotetext{
${ }^{7}$ As discussed earlier, the pairwise correlation between Child Marriage and teenage pregnancy is 0.662 ; this is shown in Table A1.
} 
In Columns 5 to 8, I repeat the analysis using drought and flood shocks across age categories 12 to 13,14 to 15 and 16 to 17 as instruments. The results remain very similar, without the teenage pregnancy control (column 5)—Child Marriage unions statistically and significantly increase stunting. From the addition of the control, the estimate is similar in magnitude but becomes statistically insignificant, due to the substantial increase in the standard error.

Although the high multicollinearity between the Child Marriage and teenage pregnancy variable interferes with the statistical power of the estimates, the coefficients remain fairly similar in magnitude between specifications with and without the control. These results indicate that Child Marriage has a direct and substantial positive effect on stunting.

\section{Instruments Diagnostics}

Statistical tests with respect to using the average of drought shock and flood shocks as instruments are presented in columns 1 to 3 of Table 1.5 . In columns 4 to 6 , the corresponding diagnostics using drought and flood shocks across age categories 12 to 13 , 14 to 15 , and 16 to 17 as instruments are reported.

One of the key assumptions of using rainfall shocks as instruments is that they are distributed independently of the error process. The Sargan statistic is used to test whether the instruments in the specifications are exogenous. Without year fixed effects, the Sargan $\mathrm{p}$-values for both sets of instruments are far from rejection of its null, lending confidence to the validity of the instruments (columns 1,3). With year fixed effects, the Sargan p- 
values are relatively lower; this is expected as some correlation may be introduced between the instruments.

Overall, the Sargan statistics confirm the validity of using the average of drought and flood shocks as instruments, failing to reject its null at the 10 percent significance level (columns 1,2) and at the 5 percent significance level (column 3). However, the low Sargan p-value reported in column 6 calls into question whether using drought and flood shocks across age categories 12 to 13,14 to 15 , and 16 to 17 as instruments is appropriate.

To evaluate how strong the identification is in the sample, I use the Cragg-Donald F statistic. Using the averages of drought and flood shocks as instruments, the size distortion in all specifications (columns 1, 2, 3) is very small, at less than 10 percent.

For the specifications using the drought shocks and flood shocks across different age categories as instruments, the bias is relatively small, at less than 5 percent of OLS (columns 4, 5). With the addition of the control for teenage pregnancy in column 6 , the Cragg Donald F statistic declines to 10.06 . The critical value for a 10 percent and 20 percent maximal relative bias is 11.12 and 6.76 , respectively. This suggests a weak instrument problem, as the bias is less than 20 percent of OLS.

Consequently, from the results of the Sargan p-value and the Cragg-Donald F statistic, I can conclude using the averages of drought and flood shocks as instruments is more efficient.

\subsection{Robustness Checks}

I perform additional analyses to examine whether the main results are robust to various issues of weather shock definition, instrument choice and different levels of stunting. 


\section{Weather Shock Definition}

The sensitivity of results to the definition of rainfall shocks is now examined. Previously, a binary indicator was used for whether a particular year held a drought or flood shock. A drought (flood) shock was defined as a year with rainfall less than (more than) 1.5 standard deviations below (above) the historical mean in each cluster. I construct a different measure of rainfall shocks using a continuous indicator. The rainfall shock is defined as the absolute value of the rainfall deviation from the historical mean in each cluster; this is similar to the construction of rainfall shocks as used by Corno and Voena (2015).

Table 1.6 presents first-stage regression results. Using an absolute measure does not take into account the differing effects of a drought and flood shock on the probability to marry before 18 . As such, rainfall shocks are negatively correlated with Child Marriage.

Table 1.7 reports results from IV regressions. Two different sets of instruments are used - the average of rainfall shocks across ages 12 to 17 in columns 1 to 3 and rainfall shocks at specific ages $(12,13,14,15,16$ and 17$)$ in columns 4 to 6 . The estimates on Child Marriage display the same pattern as in the main results. The addition of the teenage pregnancy control increases the standard error and causes the coefficients to decrease in statistical significance. I find that the Child Marriage estimate presented in column 6 does not differ using this alternative measure of rainfall. The estimated coefficient on Child Marriage using the average of rainfall shocks as an instrument (column 3), is similar, though slightly larger, and remains statistically significant at the 10 percent level. 


\section{Choice of Instruments}

In the main analysis, flood shocks and drought shocks were used as instruments. Flood shocks were found to be more highly correlated with Child Marriage than drought shocks. This can potentially be problematic as including irrelevant instruments can adversely affect estimators and increase biases. To alleviate potential concerns over this, the model is reestimated using only flood shocks as instruments for Child Marriage. IV estimates with and without fixed effects are presented in Table 1.9. In columns 1 to 3, the average effect of flood shocks across ages 12 to 17 is used as an instrument. Corresponding results from first-stage regressions are presented in Table 1.8.

The estimated coefficient on Child Marriage from the IV specification is nearly identical to the main results (column 3 in Table 1.5) and is still positive and statistically significant. This confirms that the inclusion of drought shocks an as instrument is not adversely affecting the estimate on Child Marriage.

\section{Levels of Stunting}

I also examine the severity of stunting to analyze whether children are more moderately or severely stunted from Child Marriage. Children are considered to be moderately stunted if their height-for-age $\mathrm{z}$ score is between $-2 \mathrm{SD}$ and $-3 \mathrm{SD}$ from the reference median, and severely stunted if their height-for-age z score falls below -3SD. Using these definitions, I construct indicators for whether the child in each cluster suffers from moderate or severe stunting. The results, using the average of drought and flood shocks as instruments, are 
reported in Table $1.10^{8}$. I find that when accounting for the different levels of stunting, children from Child Marriage unions are more likely to be severely stunted. The estimated coefficient of Child Marriage indicates an 8.2 percentage point increase in severe stunting, and is statistically significant at the 5 percent level. The coefficient of Child Marriage on moderate stunting is not statistically different from zero. This implies that the predominant effect of Child Marriage on children's health outcomes is concentrated on increases in severe stunting.

\subsection{Conclusion}

Bangladesh has the fourth-highest rate of Child Marriage in the world. While previous studies are forthcoming about the detrimental effects on the development of the child bride, very little is known about how the outcomes of children are impacted by Child Marriage unions. In this paper, I provide empirical evidence showing that Child Marriage adversely affects the health outcomes of children.

In an effort to disentangle the causal effect of Child Marriage from the effects of other factors on children's health outcomes, I use the random variation in rainfall to study how drought and flood shocks to income affect Child Marriage. I find that drought and flood shocks generate significant changes in the probability of Child Marriage. To further explain these changes, I provide a simple model to show that the parents' decision to enter their daughter into a Child Marriage union is driven by the differences in the type of income shocks that households are exposed to.

\footnotetext{
${ }^{8}$ OLS results are presented in Table A2.
} 
Using an anthropometric indicator as a measure of health outcomes, I find that children born from Child Marriage unions are more likely to be stunted. Furthermore, the predominant effect of Child Marriage on children's health outcomes is concentrated through increases in severe stunting.

These findings provide causal evidence that the impact of Child Marriage is not limited to the child bride, rather, it transfers to the next generation. Given the relationship between stunting and lower educational attainment in childhood and its impact on labor productivity in adulthood, Child Marriage is a discernible threat to future human capital and economic productivity in Bangladesh. From a policy perspective, these findings also highlight the need for an expansion and provision of resources directed towards children born from Child Marriage unions. Additionally, the paper contributes to an understanding of the relationship between rainfall shocks and the incidence of Child Marriage within the context of Bangladesh. 
Figure 1.1: Age at Marriage

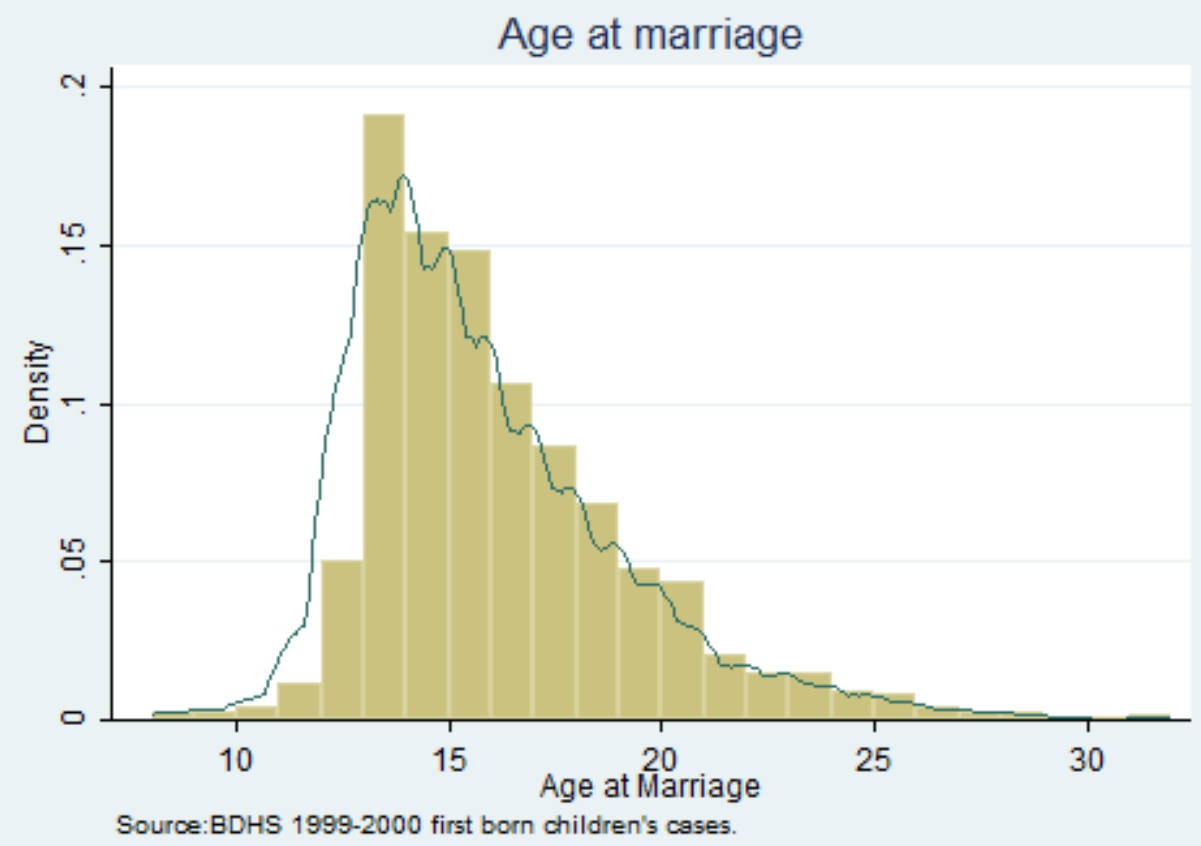


Figure 1.2 : Effect of Rainfall Shocks on Rice Yields

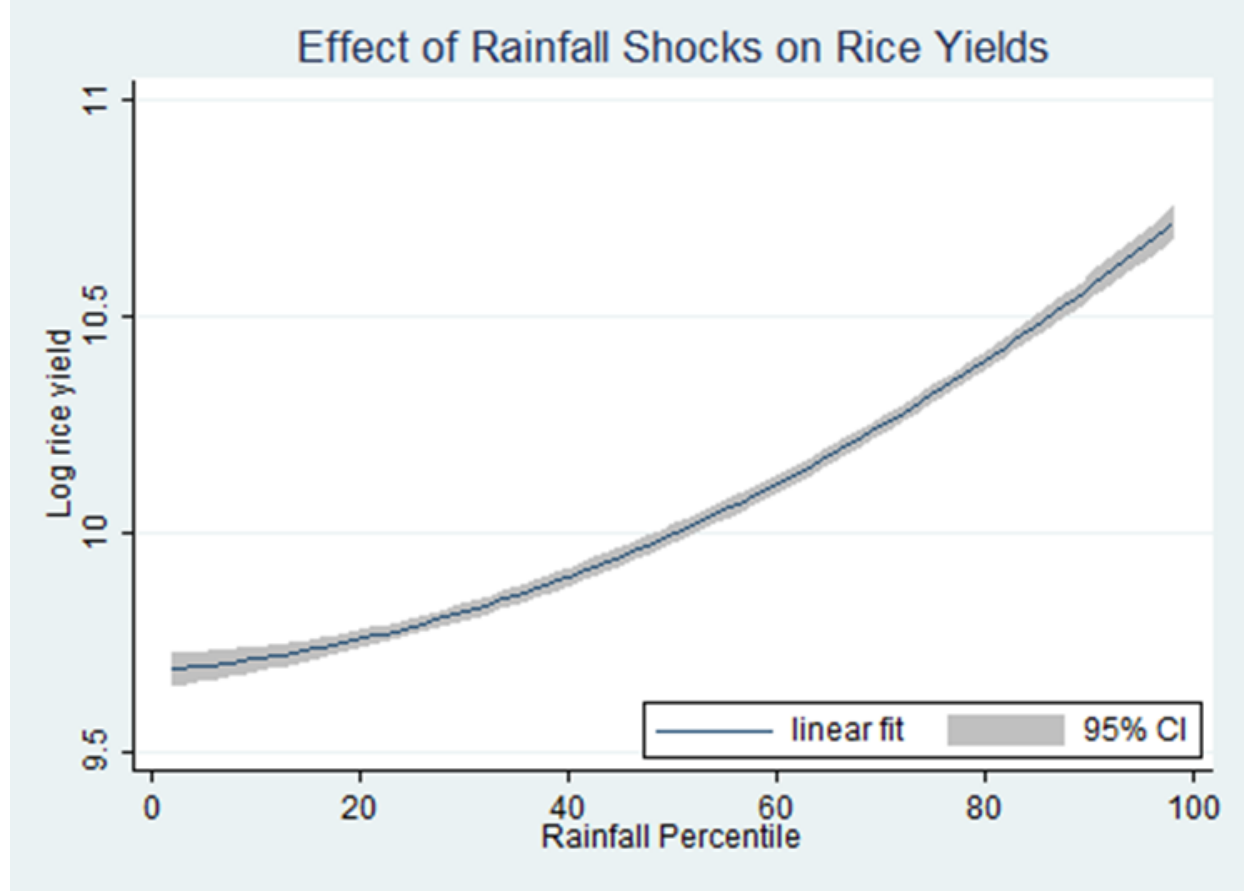


Figure 1.3: Effect of Rainfall Shocks on Wheat Yields

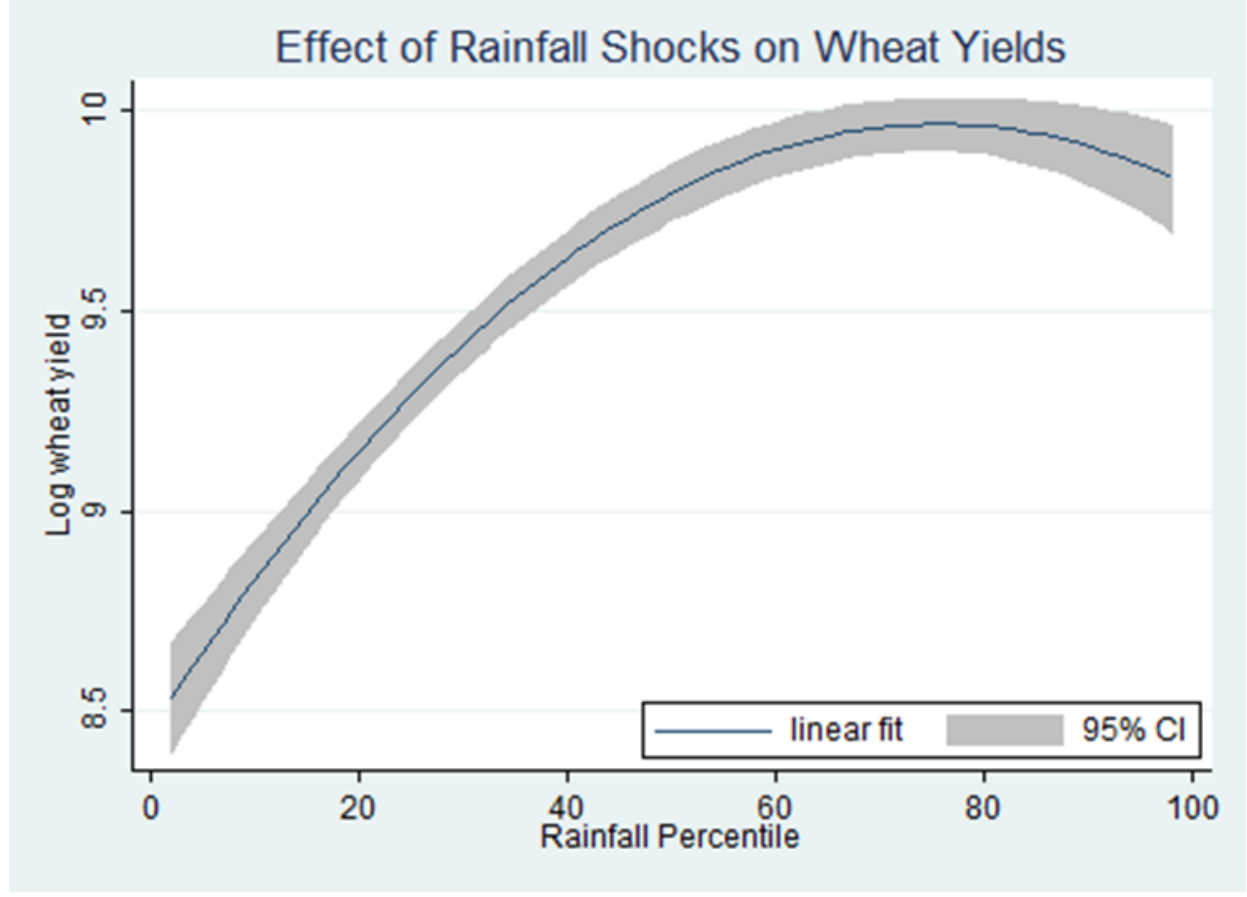


Table 1.1: Summary Statistics

\begin{tabular}{lcccc}
\hline & Mean & Std. Dev & Min & Max \\
\hline Marriage Characteristics & & & & \\
Age at Marriage & 15.81 & 3.28 & 8.00 & 32.00 \\
Married before 15 & 0.42 & 0.49 & 0.00 & 1.00 \\
Child Marriage & 0.76 & 0.43 & 0.00 & 1.00 \\
& & & & \\
Birth Characteristics & & & & \\
Age of mother at Birth & 18.76 & 3.69 & 13.00 & 40.00 \\
Mother<18 at Birth & 0.45 & 0.50 & 0.00 & 1.00 \\
& & & & \\
Health Outcomes & & & & \\
Stunted & 0.48 & 0.50 & 0.00 & 1.00 \\
Moderately Stunted & 0.29 & 0.45 & 0.00 & 1.00 \\
Severely Stunted & 0.19 & 0.39 & 0.00 & 1.00 \\
& & & & \\
Demographic Characteristics & & & & \\
Urban & 0.29 & 0.45 & 0.00 & 1.00 \\
Mother- Primary Education & 0.72 & 0.45 & 0.00 & 1.00 \\
Mother-Literate & 0.50 & 0.50 & 0.00 & 1.00 \\
Father- Primary Education & 0.64 & 0.48 & 0.00 & 1.00 \\
Lowest Quintile & 0.19 & 0.40 & 0.00 & 1.00 \\
Second Quintile & 0.17 & 0.37 & 0.00 & 1.00 \\
Fourth Quintile & 0.19 & 0.39 & 0.00 & 1.00 \\
Highest Quintile & 0.26 & 0.44 & 0.00 & 1.00 \\
& & & & \\
Rainfall Shocks & & & & \\
Flood Shock at Birth & 0.01 & 0.05 & 0.00 & 0.33 \\
Drought shock, age 12 & 0.15 & 0.36 & 0.00 & 1.00 \\
Drought shock, age 13 & 0.16 & 0.37 & 0.00 & 1.00 \\
Drought shock, age 14 & 0.12 & 0.33 & 0.00 & 1.00 \\
Drought shock, age 15 & 0.11 & 0.31 & 0.00 & 1.00 \\
Drought shock, age 16 & 0.09 & 0.28 & 0.00 & 1.00 \\
Drought shock, age 17 & 0.07 & 0.25 & 0.00 & 1.00 \\
Flood shock, age 12 & 0.05 & 0.22 & 0.00 & 1.00 \\
Flood shock, age 13 & 0.04 & 0.20 & 0.00 & 1.00 \\
Flood shock, age 14 & 0.04 & 0.19 & 0.00 & 1.00 \\
Flood shock, age 15 & 0.02 & 0.14 & 0.00 & 1.00 \\
Flood shock, age 16 & 0.02 & 0.14 & 0.00 & 1.00 \\
Flood shock, age 17 & 0.02 & 0.14 & 0.00 & 1.00 \\
\hline Observations & 1555 & & & \\
\hline & & & &
\end{tabular}


Table 1.2: Effect of Rainfall Shocks on Crop Yields

\begin{tabular}{lccc}
\hline & $(1)$ & $(2)$ & $(3)$ \\
& Rice & Wheat & Total Cereals \\
\hline Drought Shock & -0.142 & -0.108 & -0.143 \\
& $(0.232)$ & $(0.337)$ & $(0.228)$ \\
Flood shock & & & \\
& 0.0227 & 0.109 & 0.0261 \\
R2 & $(0.167)$ & $(0.244)$ & $(0.164)$ \\
Observations & 0.00857 & 0.00682 & 0.00915 \\
& 50 & 50 & 50 \\
\hline
\end{tabular}

Standard errors in parentheses

FAOSTAT Data. The dependent variable is the log of annual yield (hectograms per hectare) from 1960 to 2010

$* \mathrm{p}<0.10, * * \mathrm{p}<0.05, * * * \mathrm{p}<0.010$ 
Table 1.3: OLS Results - Effect of Child Marriage on Stunting

\begin{tabular}{|c|c|c|c|}
\hline & $\begin{array}{l}(1) \\
\text { OLS }\end{array}$ & $\begin{array}{c}(2) \\
\text { OLS }\end{array}$ & $\begin{array}{c}(3) \\
\text { OLS }\end{array}$ \\
\hline Child Marriage & $\begin{array}{c}0.0195 * * * \\
(0.00633)\end{array}$ & $\begin{array}{c}0.0173 * * * \\
(0.00618)\end{array}$ & $\begin{array}{c}0.0140^{*} \\
(0.00782)\end{array}$ \\
\hline Urban & $\begin{array}{c}-0.0171 \\
(0.0306)\end{array}$ & $\begin{array}{c}-0.0187 \\
(0.0298)\end{array}$ & $\begin{array}{c}-0.0187 \\
(0.0298)\end{array}$ \\
\hline Mother Primary Edu & $\begin{array}{c}-0.0174 \\
(0.0359)\end{array}$ & $\begin{array}{c}-0.00270 \\
(0.0351)\end{array}$ & $\begin{array}{c}-0.00388 \\
(0.0351)\end{array}$ \\
\hline Mother Literate & $\begin{array}{c}-0.0502 \\
(0.0348)\end{array}$ & $\begin{array}{l}-0.0508 \\
(0.0340)\end{array}$ & $\begin{array}{c}-0.0509 \\
(0.0340)\end{array}$ \\
\hline Father Primary Edu & $\begin{array}{c}-0.114 * * * \\
(0.0301)\end{array}$ & $\begin{array}{c}-0.116^{* * *} \\
(0.0294)\end{array}$ & $\begin{array}{c}-0.117 * * * \\
(0.0295)\end{array}$ \\
\hline Lowest Quintile & $\begin{array}{c}0.0199 \\
(0.0412)\end{array}$ & $\begin{array}{l}0.00447 \\
(0.0403)\end{array}$ & $\begin{array}{c}0.00432 \\
(0.0403)\end{array}$ \\
\hline Second Quintile & $\begin{array}{c}-0.0278 \\
(0.0413)\end{array}$ & $\begin{array}{c}-0.0388 \\
(0.0403)\end{array}$ & $\begin{array}{c}-0.0393 \\
(0.0403)\end{array}$ \\
\hline Fourth Quintile & $\begin{array}{l}-0.0780 * \\
(0.0404)\end{array}$ & $\begin{array}{c}-0.0933 * * \\
(0.0395)\end{array}$ & $\begin{array}{c}-0.0915 * * \\
(0.0396)\end{array}$ \\
\hline Highest Quintile & $\begin{array}{c}-0.169 * * * \\
(0.0425)\end{array}$ & $\begin{array}{c}-0.183 * * * \\
(0.0416)\end{array}$ & $\begin{array}{c}-0.181 * * * \\
(0.0417)\end{array}$ \\
\hline Flood Shock at Birth & $\begin{array}{l}-0.430 \\
(0.271)\end{array}$ & $\begin{array}{c}-0.489^{*} \\
(0.274)\end{array}$ & $\begin{array}{l}-0.487^{*} \\
(0.274)\end{array}$ \\
\hline Teenage Pregnancy & & & $\begin{array}{c}0.0218 \\
(0.0319) \\
\end{array}$ \\
\hline FE & no & yes & yes \\
\hline $\mathrm{R} 2$ & 0.0947 & 0.0957 & 0.0959 \\
\hline $\mathrm{F}$ & 16.14 & 16.27 & 14.83 \\
\hline Observations & 1555 & 1555 & 1555 \\
\hline
\end{tabular}

Standard errors in parentheses

1999-2000 DHS Data. OLS regression; dependent variable is children's health outcomes measured by stunting.

$* \mathrm{p}<0.10,{ }^{* *} \mathrm{p}<0.05,{ }^{* * *} \mathrm{p}<0.010$ 
Table 1.4: First-stage IV Results - Effect of Child Marriage on Stunting

\begin{tabular}{|c|c|c|c|c|c|c|}
\hline & $\begin{array}{l}(1) \\
\text { CM }\end{array}$ & $\begin{array}{l}(2) \\
\mathrm{CM} \\
\end{array}$ & $\begin{array}{l}(3) \\
\text { CM }\end{array}$ & $\begin{array}{l}\text { (4) } \\
\text { CM }\end{array}$ & $\begin{array}{c}(5) \\
\mathrm{CM} \\
\end{array}$ & $\begin{array}{c}\text { (6) } \\
\text { CM }\end{array}$ \\
\hline Drought shock & $\begin{array}{c}-2.862 * * * \\
(0.382)\end{array}$ & $\begin{array}{c}-3.286^{* * *} \\
(0.390)\end{array}$ & $\begin{array}{c}-0.0658 \\
(0.353)\end{array}$ & & & \\
\hline Flood shock & $\begin{array}{c}-8.144 * * * \\
(0.561)\end{array}$ & $\begin{array}{c}-8.672 * * * \\
(0.569)\end{array}$ & $\begin{array}{c}-3.293 * * * \\
(0.525)\end{array}$ & & & \\
\hline Urban & $\begin{array}{c}-0.257 * * \\
(0.115)\end{array}$ & $\begin{array}{c}-0.258 * * \\
(0.114)\end{array}$ & $\begin{array}{c}-0.149 \\
(0.0961)\end{array}$ & $\begin{array}{c}-0.251 * * \\
(0.112)\end{array}$ & $\begin{array}{c}-0.251 * * \\
(0.111)\end{array}$ & $\begin{array}{c}-0.152 \\
(0.0957)\end{array}$ \\
\hline Mother Primary Edu & $\begin{array}{l}0.0980 \\
(0.135)\end{array}$ & $\begin{array}{c}0.120 \\
(0.134)\end{array}$ & $\begin{array}{c}-0.0302 \\
(0.113)\end{array}$ & $\begin{array}{c}0.109 \\
(0.132)\end{array}$ & $\begin{array}{c}0.135 \\
(0.131)\end{array}$ & $\begin{array}{l}-0.0131 \\
(0.113)\end{array}$ \\
\hline Mother Literate & $\begin{array}{c}-0.644 * * * \\
(0.129)\end{array}$ & $\begin{array}{c}-0.619 * * * \\
(0.129)\end{array}$ & $\begin{array}{c}-0.450 * * * \\
(0.109)\end{array}$ & $\begin{array}{c}-0.652 * * * \\
(0.127)\end{array}$ & $\begin{array}{c}-0.629 * * * \\
(0.126)\end{array}$ & $\begin{array}{c}-0.476^{* * *} \\
(0.109)\end{array}$ \\
\hline Father Primary Edu & $\begin{array}{c}-0.323 * * * \\
(0.113)\end{array}$ & $\begin{array}{c}-0.292 * * * \\
(0.112)\end{array}$ & $\begin{array}{c}-0.277 * * * \\
(0.0945)\end{array}$ & $\begin{array}{c}-0.299 * * * \\
(0.110)\end{array}$ & $\begin{array}{c}-0.262^{* *} \\
(0.110)\end{array}$ & $\begin{array}{c}-0.262 * * * \\
(0.0942)\end{array}$ \\
\hline Lowest Quintile & $\begin{array}{c}0.132 \\
(0.155)\end{array}$ & $\begin{array}{c}0.128 \\
(0.154)\end{array}$ & $\begin{array}{l}0.0494 \\
(0.130)\end{array}$ & $\begin{array}{l}0.0812 \\
(0.151)\end{array}$ & $\begin{array}{l}0.0793 \\
(0.150)\end{array}$ & $\begin{array}{l}0.0302 \\
(0.129)\end{array}$ \\
\hline Second Quintile & $\begin{array}{c}0.126 \\
(0.155)\end{array}$ & $\begin{array}{c}0.109 \\
(0.154)\end{array}$ & $\begin{array}{l}0.0124 \\
(0.130)\end{array}$ & $\begin{array}{c}0.145 \\
(0.151)\end{array}$ & $\begin{array}{c}0.131 \\
(0.150)\end{array}$ & $\begin{array}{l}0.0214 \\
(0.129)\end{array}$ \\
\hline Fourth Quintile & $\begin{array}{c}-0.670 * * * \\
(0.150)\end{array}$ & $\begin{array}{c}-0.694 * * * \\
(0.150)\end{array}$ & $\begin{array}{c}-0.315^{* *} \\
(0.127)\end{array}$ & $\begin{array}{c}-0.649 * * * \\
(0.147)\end{array}$ & $\begin{array}{c}-0.671 * * * \\
(0.146)\end{array}$ & $\begin{array}{c}-0.316 * * \\
(0.127)\end{array}$ \\
\hline Highest Quintile & $\begin{array}{c}-0.970 * * * \\
(0.157)\end{array}$ & $\begin{array}{c}-0.989 * * * \\
(0.157)\end{array}$ & $\begin{array}{c}-0.509 * * * \\
(0.134)\end{array}$ & $\begin{array}{c}-0.929 * * * \\
(0.154)\end{array}$ & $\begin{array}{c}-0.942 * * * \\
(0.153)\end{array}$ & $\begin{array}{c}-0.511 * * * \\
(0.133)\end{array}$ \\
\hline Flood Shock at Birth & $\begin{array}{c}5.636^{* * * *} \\
(1.039)\end{array}$ & $\begin{array}{c}5.185 * * * \\
(1.059)\end{array}$ & $\begin{array}{c}2.615^{* * *} \\
(0.898)\end{array}$ & $\begin{array}{c}5.712 * * * \\
(1.016)\end{array}$ & $\begin{array}{c}5.192 * * * \\
(1.034)\end{array}$ & $\begin{array}{c}2.623 * * * \\
(0.897)\end{array}$ \\
\hline Teenage Pregnancy & & & $\begin{array}{c}2.307 * * * \\
(0.0919)\end{array}$ & & & $\begin{array}{c}2.261 * * * \\
(0.0974)\end{array}$ \\
\hline Drought shock, 12 to 13 & & & & $\begin{array}{l}0.0335 \\
(0.178)\end{array}$ & $\begin{array}{c}-0.0830 \\
(0.178)\end{array}$ & $\begin{array}{c}0.113 \\
(0.153)\end{array}$ \\
\hline Drought shock, 14 to 15 & & & & $\begin{array}{c}-1.198 * * * \\
(0.182)\end{array}$ & $\begin{array}{c}-1.369^{* * *} * \\
(0.183)\end{array}$ & $\begin{array}{c}0.134 \\
(0.170)\end{array}$ \\
\hline Drought shock, 16 to 17 & & & & $\begin{array}{c}-2.180 * * * \\
(0.231)\end{array}$ & $\begin{array}{c}-2.394 * * * \\
(0.233)\end{array}$ & $\begin{array}{c}-0.540 * * \\
(0.215)\end{array}$ \\
\hline Flood shock, 12 to 13 & & & & $\begin{array}{c}-2.520 * * * \\
(0.289)\end{array}$ & $\begin{array}{c}-2.731 * * * \\
(0.289)\end{array}$ & $\begin{array}{c}-1.389 * * * \\
(0.255)\end{array}$ \\
\hline Flood shock, 14 to 15 & & & & $\begin{array}{c}-2.458 * * * \\
(0.388)\end{array}$ & $\begin{array}{c}-2.602 * * * \\
(0.387)\end{array}$ & $\begin{array}{c}-0.453 \\
(0.345)\end{array}$ \\
\hline
\end{tabular}




\begin{tabular}{lcccccc} 
Flood shock,16 to 17 & & & $-2.434^{* * *}$ & $-2.628^{* * *}$ & $-1.029 * * *$ \\
& & & & $(0.415)$ & $(0.413)$ & $(0.362)$ \\
\hline FE & no & yes & yes & no & yes & yes \\
R2 & 0.297 & 0.306 & 0.508 & 0.330 & 0.342 & 0.513 \\
F & 59.25 & 61.70 & 132.2 & 50.53 & 53.18 & 101.0 \\
Observations & 1555 & 1555 & 1555 & 1555 & 1555 & 1555 \\
\hline
\end{tabular}

Standard errors in parentheses

1999-2000 DHS Data. OLS regression; dependent variable is Child Marriage.

$* \mathrm{p}<0.10,{ }^{* *} \mathrm{p}<0.05,{ }^{* * *} \mathrm{p}<0.010$ 
Table 1.5: IV Results - Effect of Child Marriage on Stunting

\begin{tabular}{|c|c|c|c|c|c|c|}
\hline & $\begin{array}{l}\text { (1) } \\
\text { IV }\end{array}$ & $\begin{array}{l}\text { (2) } \\
\text { IV }\end{array}$ & $\begin{array}{l}\text { (3) } \\
\text { IV }\end{array}$ & $\begin{array}{l}\text { (4) } \\
\text { IV }\end{array}$ & $\begin{array}{l}\text { (5) } \\
\text { IV }\end{array}$ & $\begin{array}{l}\text { (6) } \\
\text { IV }\end{array}$ \\
\hline Child Marriage & $\begin{array}{c}0.0385 * * \\
(0.0175)\end{array}$ & $\begin{array}{c}0.0516^{* * * *} \\
(0.0164)\end{array}$ & $\begin{array}{l}0.0833^{*} \\
(0.0480)\end{array}$ & $\begin{array}{l}0.0272 * \\
(0.0152)\end{array}$ & $\begin{array}{c}0.0388 * * * \\
(0.0143)\end{array}$ & $\begin{array}{c}0.0349 \\
(0.0401)\end{array}$ \\
\hline Urban & $\begin{array}{c}-0.0128 \\
(0.0308)\end{array}$ & $\begin{array}{c}-0.0108 \\
(0.0302)\end{array}$ & $\begin{array}{c}-0.00848 \\
(0.0313)\end{array}$ & $\begin{array}{c}-0.0153 \\
(0.0306)\end{array}$ & $\begin{array}{c}-0.0138 \\
(0.0300)\end{array}$ & $\begin{array}{l}-0.0157 \\
(0.0304)\end{array}$ \\
\hline Mother Primary Edu & $\begin{array}{c}-0.0210 \\
(0.0360)\end{array}$ & $\begin{array}{l}-0.00979 \\
(0.0354)\end{array}$ & $\begin{array}{c}-0.00341 \\
(0.0359)\end{array}$ & $\begin{array}{c}-0.0189 \\
(0.0359)\end{array}$ & $\begin{array}{c}-0.00715 \\
(0.0352)\end{array}$ & $\begin{array}{c}-0.00374 \\
(0.0351)\end{array}$ \\
\hline Mother Literate & $\begin{array}{l}-0.0367 \\
(0.0367)\end{array}$ & $\begin{array}{l}-0.0267 \\
(0.0359)\end{array}$ & $\begin{array}{c}-0.0194 \\
(0.0409)\end{array}$ & $\begin{array}{l}-0.0447 \\
(0.0361)\end{array}$ & $\begin{array}{c}-0.0357 \\
(0.0352)\end{array}$ & $\begin{array}{l}-0.0415 \\
(0.0384)\end{array}$ \\
\hline Father Primary Edu & $\begin{array}{c}0.107 * * * \\
(0.0307)\end{array}$ & $\begin{array}{c}-0.104 * * * \\
(0.0301)\end{array}$ & $\begin{array}{c}0.0963 * * * \\
(0.0331)\end{array}$ & $\begin{array}{c}-0.111 * * * \\
(0.0305)\end{array}$ & $\begin{array}{c}-0.108 * * * \\
(0.0298)\end{array}$ & $\begin{array}{c}-0.110^{* * *} \\
(0.0316)\end{array}$ \\
\hline Lowest Quintile & $\begin{array}{c}0.0182 \\
(0.0412)\end{array}$ & $\begin{array}{l}0.00179 \\
(0.0406)\end{array}$ & $\begin{array}{l}0.00214 \\
(0.0412)\end{array}$ & $\begin{array}{c}0.0192 \\
(0.0411)\end{array}$ & $\begin{array}{l}0.00279 \\
(0.0404)\end{array}$ & $\begin{array}{l}0.00366 \\
(0.0403)\end{array}$ \\
\hline Second Quintile & $\begin{array}{c}-0.0303 \\
(0.0413)\end{array}$ & $\begin{array}{l}-0.0430 \\
(0.0406)\end{array}$ & $\begin{array}{l}-0.0405 \\
(0.0412)\end{array}$ & $\begin{array}{c}-0.0288 \\
(0.0412)\end{array}$ & $\begin{array}{c}-0.0415 \\
(0.0404)\end{array}$ & $\begin{array}{c}-0.0397 \\
(0.0403)\end{array}$ \\
\hline Fourth Quintile & $\begin{array}{c}-0.0627 \\
(0.0424)\end{array}$ & $\begin{array}{c}-0.0653 \\
(0.0417)\end{array}$ & $\begin{array}{l}-0.0702 \\
(0.0430)\end{array}$ & $\begin{array}{l}-0.0718^{*} \\
(0.0417)\end{array}$ & $\begin{array}{c}-0.0757^{*} \\
(0.0409)\end{array}$ & $\begin{array}{c}-0.0851^{* *} \\
(0.0414)\end{array}$ \\
\hline Highest Quintile & $\begin{array}{c}-0.145^{* * *} \\
(0.0471)\end{array}$ & $\begin{array}{c}-0.140^{* * *} \\
(0.0461)\end{array}$ & $\begin{array}{c}-0.144^{* * *} \\
(0.0496)\end{array}$ & $\begin{array}{c}-0.159 * * * \\
(0.0457)\end{array}$ & $\begin{array}{c}-0.156^{* * *} \\
(0.0447)\end{array}$ & $\begin{array}{c}-0.170 * * * \\
(0.0467)\end{array}$ \\
\hline Flood Shock at Birth & $\begin{array}{c}-0.493^{*} \\
(0.277)\end{array}$ & $\begin{array}{c}-0.606^{* *} \\
(0.280)\end{array}$ & $\begin{array}{c}-0.655^{* *} \\
(0.302)\end{array}$ & $\begin{array}{l}-0.455^{*} \\
(0.274)\end{array}$ & $\begin{array}{c}-0.562 * * \\
(0.277)\end{array}$ & $\begin{array}{c}-0.537^{*} \\
(0.290)\end{array}$ \\
\hline Teenage Pregnancy & & & $\begin{array}{c}-0.151 \\
(0.123)\end{array}$ & & & $\begin{array}{r}-0.0302 \\
(0.103)\end{array}$ \\
\hline FE & no & yes & yes & no & yes & yes \\
\hline R2 & 0.0894 & 0.0775 & 0.0498 & 0.0938 & 0.0885 & 0.0918 \\
\hline Sargan p-value & 0.915 & 0.528 & 0.0964 & 0.169 & 0.0824 & 0.0361 \\
\hline Cragg-Donald F & 116.3 & 128.8 & 21.90 & 53.23 & 59.12 & 10.06 \\
\hline Observations & 1555 & 1555 & 1555 & 1555 & 1555 & 1555 \\
\hline
\end{tabular}

Standard errors in parentheses

1999-2000 DHS Data. Columns 1 to 3 use the average effect of drought shocks and flood shocks across ages 12 to 17 as instruments. Columns 4 to 6 use drought shocks and flood shocks across age categories 12 to 13,14 to 15,16 to 17 as instruments. The dependent variable is children's health outcomes, measured by stunting.

$* \mathrm{p}<0.10, * * \mathrm{p}<0.05, * * * \mathrm{p}<0.010$ 
Table 1.6: First-stage IV Results - Weather Shock Definition

\begin{tabular}{|c|c|c|c|c|c|c|}
\hline & $\begin{array}{l}(1) \\
\mathrm{CM}\end{array}$ & $\begin{array}{l}(2) \\
\mathrm{CM}\end{array}$ & $\begin{array}{l}(3) \\
\mathrm{CM}\end{array}$ & $\begin{array}{l}(4) \\
\mathrm{CM}\end{array}$ & $\begin{array}{l}(5) \\
\mathrm{CM}\end{array}$ & $\begin{array}{l}(6) \\
\mathrm{CM}\end{array}$ \\
\hline Rainfall shock & $\begin{array}{c}-2.617 * * * \\
(0.177)\end{array}$ & $\begin{array}{c}-2.884 * * * \\
(0.183)\end{array}$ & $\begin{array}{c}-0.680^{* * *} \\
(0.179)\end{array}$ & & & \\
\hline Urban & $\begin{array}{l}-0.188 \\
(0.115)\end{array}$ & $\begin{array}{l}-0.180 \\
(0.114)\end{array}$ & $\begin{array}{c}-0.142 \\
(0.0968)\end{array}$ & $\begin{array}{c}-0.182 \\
(0.113)\end{array}$ & $\begin{array}{l}-0.173 \\
(0.112)\end{array}$ & $\begin{array}{c}-0.125 \\
(0.0963)\end{array}$ \\
\hline Mother Primary Edu & $\begin{array}{l}0.0925 \\
(0.135)\end{array}$ & $\begin{array}{c}0.115 \\
(0.134)\end{array}$ & $\begin{array}{l}-0.0131 \\
(0.114)\end{array}$ & $\begin{array}{c}0.104 \\
(0.132)\end{array}$ & $\begin{array}{c}0.127 \\
(0.131)\end{array}$ & $\begin{array}{c}0.00683 \\
(0.113)\end{array}$ \\
\hline Mother Literate & $\begin{array}{c}-0.591 * * * \\
(0.130)\end{array}$ & $\begin{array}{c}-0.563 * * * \\
(0.129)\end{array}$ & $\begin{array}{c}-0.439 * * * \\
(0.110)\end{array}$ & $\begin{array}{c}-0.608 * * * \\
(0.128)\end{array}$ & $\begin{array}{c}-0.580 * * * \\
(0.127)\end{array}$ & $\begin{array}{c}-0.470 * * * \\
(0.109)\end{array}$ \\
\hline Father Primary Edu & $\begin{array}{c}-0.322 * * * \\
(0.113)\end{array}$ & $\begin{array}{c}-0.289^{* *} \\
(0.112)\end{array}$ & $\begin{array}{c}-0.281 * * * \\
(0.0954)\end{array}$ & $\begin{array}{c}-0.330^{* * *} \\
(0.111)\end{array}$ & $\begin{array}{c}-0.293 * * * \\
(0.110)\end{array}$ & $\begin{array}{c}-0.274 * * * \\
(0.0948)\end{array}$ \\
\hline Lowest Quintile & $\begin{array}{c}0.115 \\
(0.155)\end{array}$ & $\begin{array}{c}0.113 \\
(0.154)\end{array}$ & $\begin{array}{l}0.0431 \\
(0.131)\end{array}$ & $\begin{array}{c}0.102 \\
(0.152)\end{array}$ & $\begin{array}{c}0.106 \\
(0.151)\end{array}$ & $\begin{array}{l}0.0540 \\
(0.130)\end{array}$ \\
\hline Second Quintile & $\begin{array}{c}0.115 \\
(0.155)\end{array}$ & $\begin{array}{l}0.0981 \\
(0.154)\end{array}$ & $\begin{array}{l}0.0191 \\
(0.131)\end{array}$ & $\begin{array}{c}0.111 \\
(0.152)\end{array}$ & $\begin{array}{l}0.0945 \\
(0.151)\end{array}$ & $\begin{array}{c}0.0261 \\
(0.130)\end{array}$ \\
\hline Fourth Quintile & $\begin{array}{c}-0.714 * * * \\
(0.151)\end{array}$ & $\begin{array}{c}-0.737 * * * \\
(0.150)\end{array}$ & $\begin{array}{c}-0.325^{* *} \\
(0.128)\end{array}$ & $\begin{array}{c}-0.673^{* * *} \\
(0.148)\end{array}$ & $\begin{array}{c}-0.693 * * * \\
(0.147)\end{array}$ & $\begin{array}{c}-0.312 * * \\
(0.128)\end{array}$ \\
\hline Highest Quintile & $\begin{array}{c}-1.029 * * * \\
(0.158)\end{array}$ & $\begin{array}{c}-1.045^{* * *} \\
(0.157)\end{array}$ & $\begin{array}{c}-0.537 * * * \\
(0.135)\end{array}$ & $\begin{array}{c}-0.955 * * * \\
(0.155)\end{array}$ & $\begin{array}{c}-0.963 * * * \\
(0.154)\end{array}$ & $\begin{array}{c}-0.521^{* * *} \\
(0.134)\end{array}$ \\
\hline Flood shock at Birth & $\begin{array}{c}5.120 * * * \\
(1.025)\end{array}$ & $\begin{array}{c}4.645^{* * *} \\
(1.047)\end{array}$ & $\begin{array}{c}2.793 * * * \\
(0.892)\end{array}$ & $\begin{array}{c}5.069^{* * *} \\
(1.006)\end{array}$ & $\begin{array}{c}4.475^{* * *} \\
(1.026)\end{array}$ & $\begin{array}{c}2.682^{* * * *} \\
(0.887)\end{array}$ \\
\hline Teenage Pregnancy & & & $\begin{array}{c}2.317 * * * \\
(0.0945)\end{array}$ & & & $\begin{array}{c}2.282 * * * \\
(0.0988)\end{array}$ \\
\hline Rainfall shock, age 12 & & & & $\begin{array}{c}-0.141^{* *} \\
(0.0654)\end{array}$ & $\begin{array}{c}-0.170^{* * * *} \\
(0.0651)\end{array}$ & $\begin{array}{c}-0.0474 \\
(0.0563)\end{array}$ \\
\hline Rainfall shock, age 13 & & & & $\begin{array}{c}-0.311^{* * *} \\
(0.0616)\end{array}$ & $\begin{array}{c}-0.351 * * * * \\
(0.0614)\end{array}$ & $\begin{array}{c}-0.169^{* * * *} \\
(0.0535)\end{array}$ \\
\hline Rainfall shock, age 14 & & & & $\begin{array}{c}-0.375^{* * * *} \\
(0.0677)\end{array}$ & $\begin{array}{c}-0.435^{* * * *} \\
(0.0678)\end{array}$ & $\begin{array}{c}-0.00514 \\
(0.0613)\end{array}$ \\
\hline Rainfall shock, age 15 & & & & $\begin{array}{c}-0.494 * * * \\
(0.0737)\end{array}$ & $\begin{array}{c}-0.547 * * * \\
(0.0735)\end{array}$ & $\begin{array}{c}-0.0477 \\
(0.0669)\end{array}$ \\
\hline Rainfall shock, age 16 & & & & $\begin{array}{c}-0.536 * * * \\
(0.0770)\end{array}$ & $\begin{array}{c}-0.596^{* * *} \\
(0.0769)\end{array}$ & $\begin{array}{c}-0.0907 \\
(0.0698)\end{array}$ \\
\hline
\end{tabular}


Rainfall shock, age 17

\section{FE}

$\mathrm{R} 2$

$\mathrm{F}$

Observations

Standard errors in parentheses

1999-2000 DHS Data. OLS regression; dependent variable is Child Marriage- the probability of marriage before age 18.

$* \mathrm{p}<0.10, * * \mathrm{p}<0.05, * * * \mathrm{p}<0.010$ $\begin{array}{ccc}-0.899 * * * & -0.954 * * * & -0.412 * * * \\ (0.0814) & (0.0815) & (0.0741)\end{array}$

$\begin{array}{cccccc}\text { no } & \text { yes } & \text { yes } & \text { no } & \text { yes } & \text { yes } \\ 0.291 & 0.303 & 0.499 & 0.322 & 0.336 & 0.507 \\ 63.35 & 66.74 & 139.0 & 48.72 & 51.69 & 98.62 \\ 1555 & 1555 & 1555 & 1555 & 1555 & 1555\end{array}$

( 
Table 1.7: IV Results - Weather Shock Definition

\begin{tabular}{|c|c|c|c|c|c|c|}
\hline & $\begin{array}{l}\text { (1) } \\
\text { IV }\end{array}$ & (2) & (3) & (4) & (5) & $\begin{array}{l}\text { (6) } \\
\text { IV }\end{array}$ \\
\hline Child Marriage & $\begin{array}{c}0.0273 \\
(0.0180)\end{array}$ & $\begin{array}{c}0.0465 * * * \\
(0.0166)\end{array}$ & $\begin{array}{c}0.158^{*} \\
(0.0895)\end{array}$ & $\begin{array}{c}0.0320^{* *} \\
(0.0157)\end{array}$ & $\begin{array}{c}0.0471 * * * \\
(0.0146)\end{array}$ & $\begin{array}{l}0.0863^{*} \\
(0.0494)\end{array}$ \\
\hline Urban & $\begin{array}{l}-0.0153 \\
(0.0307)\end{array}$ & $\begin{array}{l}-0.0120 \\
(0.0302)\end{array}$ & $\begin{array}{l}0.00251 \\
(0.0354)\end{array}$ & $\begin{array}{l}-0.0143 \\
(0.0307)\end{array}$ & $\begin{array}{l}-0.0119 \\
(0.0301)\end{array}$ & $\begin{array}{l}-0.00804 \\
(0.0314)\end{array}$ \\
\hline Mother Primary Edu & $\begin{array}{l}-0.0189 \\
(0.0359)\end{array}$ & $\begin{array}{l}-0.00874 \\
(0.0353)\end{array}$ & $\begin{array}{l}-0.00291 \\
(0.0386)\end{array}$ & $\begin{array}{l}-0.0198 \\
(0.0359)\end{array}$ & $\begin{array}{l}-0.00886 \\
(0.0353)\end{array}$ & $\begin{array}{l}-0.00339 \\
(0.0359)\end{array}$ \\
\hline Mother Literate & $\begin{array}{l}-0.0446 \\
(0.0367)\end{array}$ & $\begin{array}{l}-0.0303 \\
(0.0358)\end{array}$ & $\begin{array}{c}0.0143 \\
(0.0551)\end{array}$ & $\begin{array}{l}-0.0413 \\
(0.0362)\end{array}$ & $\begin{array}{l}-0.0299 \\
(0.0354)\end{array}$ & $\begin{array}{l}-0.0181 \\
(0.0413)\end{array}$ \\
\hline Father Primary Edu & $\begin{array}{c}-0.111 * * * \\
(0.0306)\end{array}$ & $\begin{array}{c}-0.106 * * * \\
(0.0301)\end{array}$ & $\begin{array}{l}-0.0747 * \\
(0.0415)\end{array}$ & $\begin{array}{c}-0.110 * * * \\
(0.0305)\end{array}$ & $\begin{array}{c}-0.105 * * * \\
(0.0299)\end{array}$ & $\begin{array}{c}-0.0954 * * * \\
(0.0333)\end{array}$ \\
\hline Lowest Quintile & $\begin{array}{c}0.0192 \\
(0.0411)\end{array}$ & $\begin{array}{l}0.00219 \\
(0.0405)\end{array}$ & $\begin{array}{c}-0.000186 \\
(0.0445)\end{array}$ & $\begin{array}{c}0.0188 \\
(0.0412)\end{array}$ & $\begin{array}{l}0.00214 \\
(0.0405)\end{array}$ & $\begin{array}{l}0.00205 \\
(0.0413)\end{array}$ \\
\hline Second Quintile & $\begin{array}{l}-0.0289 \\
(0.0412)\end{array}$ & $\begin{array}{l}-0.0424 \\
(0.0405)\end{array}$ & $\begin{array}{l}-0.0418 \\
(0.0444)\end{array}$ & $\begin{array}{l}-0.0295 \\
(0.0412)\end{array}$ & $\begin{array}{l}-0.0425 \\
(0.0405)\end{array}$ & $\begin{array}{l}-0.0406 \\
(0.0413)\end{array}$ \\
\hline Fourth Quintile & $\begin{array}{l}-0.0717^{*} \\
(0.0424)\end{array}$ & $\begin{array}{l}-0.0694 * \\
(0.0416)\end{array}$ & $\begin{array}{l}-0.0474 \\
(0.0514)\end{array}$ & $\begin{array}{l}-0.0679 \\
(0.0419)\end{array}$ & $\begin{array}{l}-0.0690 * \\
(0.0411)\end{array}$ & $\begin{array}{l}-0.0693 \\
(0.0432)\end{array}$ \\
\hline Highest Quintile & $\begin{array}{c}-0.159 * * * \\
(0.0473)\end{array}$ & $\begin{array}{c}-0.146 * * * \\
(0.0461)\end{array}$ & $\begin{array}{c}-0.104 \\
(0.0663)\end{array}$ & $\begin{array}{c}-0.153 * * * \\
(0.0460)\end{array}$ & $\begin{array}{c}-0.145 * * * \\
(0.0450)\end{array}$ & $\begin{array}{c}-0.142 * * * \\
(0.0501)\end{array}$ \\
\hline Flood Shock at Birth & $\begin{array}{l}-0.456 * \\
(0.276)\end{array}$ & $\begin{array}{c}-0.589 * * \\
(0.280)\end{array}$ & $\begin{array}{c}-0.835 * * \\
(0.371)\end{array}$ & $\begin{array}{l}-0.471 * \\
(0.275)\end{array}$ & $\begin{array}{c}-0.591 * * \\
(0.279)\end{array}$ & $\begin{array}{c}-0.662 * * \\
(0.304)\end{array}$ \\
\hline Teenage Pregnancy & & & $\begin{array}{l}-0.337 \\
(0.225) \\
\end{array}$ & & & $\begin{array}{l}-0.159 \\
(0.126) \\
\end{array}$ \\
\hline $\mathrm{FE}$ & no & yes & yes & no & yes & yes \\
\hline $\mathrm{R} 2$ & 0.0938 & 0.0825 & -0.102 & 0.0924 & 0.0820 & 0.0458 \\
\hline Sargan p-value & & $\cdot$ & . & 0.891 & 0.887 & 0.552 \\
\hline Cragg-Donald F & 217.7 & 248.3 & 14.36 & 49.56 & 56.12 & 6.899 \\
\hline Observations & 1555 & 1555 & 1555 & 1555 & 1555 & 1555 \\
\hline
\end{tabular}

Standard errors in parentheses

1999-2000 DHS Data. Rainfall shocks are calculated as the absolute value of rainfall deviations from the historical mean in each cluster. Columns 1 to 3 use the average effect of rainfall shocks across ages 12 to 17 as instruments. Columns 4 to 6 use rainfall shocks at ages 12,13,14,15,16 and 17 as instruments. The dependent variable is children's health outcomes, measured by stunting

$* \mathrm{p}<0.10, * * \mathrm{p}<0.05, * * * \mathrm{p}<0.010$ 
Table 1.8: First-stage Results - Choice of Instruments

\begin{tabular}{|c|c|c|c|}
\hline & $\begin{array}{l}(1) \\
\mathrm{CM} \\
\end{array}$ & $\begin{array}{l}(2) \\
\text { CM }\end{array}$ & $\begin{array}{l}(3) \\
\mathrm{CM}\end{array}$ \\
\hline Flood shock & $\begin{array}{c}-7.312 * * * \\
(0.560)\end{array}$ & $\begin{array}{c}-7.564 * * * \\
(0.566)\end{array}$ & $\begin{array}{c}-3.259 * * * \\
(0.493)\end{array}$ \\
\hline Urban & $\begin{array}{c}-0.216^{*} \\
(0.116)\end{array}$ & $\begin{array}{c}-0.214 * \\
(0.116)\end{array}$ & $\begin{array}{c}-0.147 \\
(0.0959)\end{array}$ \\
\hline Mother Primary Edu & $\begin{array}{c}0.0992 \\
(0.137)\end{array}$ & $\begin{array}{c}0.115 \\
(0.137)\end{array}$ & $\begin{array}{l}-0.0307 \\
(0.113)\end{array}$ \\
\hline Mother Literate & $\begin{array}{c}-0.666 * * * \\
(0.132)\end{array}$ & $\begin{array}{c}-0.650 * * * \\
(0.132)\end{array}$ & $\begin{array}{c}-0.450 * * * \\
(0.109)\end{array}$ \\
\hline Father Primary Edu & $\begin{array}{c}-0.329 * * * \\
(0.115)\end{array}$ & $\begin{array}{c}-0.310 * * * \\
(0.115)\end{array}$ & $\begin{array}{c}-0.277 * * * \\
(0.0944)\end{array}$ \\
\hline Lowest Quintile & $\begin{array}{c}0.121 \\
(0.157)\end{array}$ & $\begin{array}{c}0.111 \\
(0.157)\end{array}$ & $\begin{array}{l}0.0489 \\
(0.130)\end{array}$ \\
\hline Second Quintile & $\begin{array}{c}0.108 \\
(0.157)\end{array}$ & $\begin{array}{l}0.0915 \\
(0.157)\end{array}$ & $\begin{array}{l}0.0118 \\
(0.130)\end{array}$ \\
\hline Fourth Quintile & $\begin{array}{c}-0.722 * * * \\
(0.153)\end{array}$ & $\begin{array}{c}-0.748 * * * \\
(0.153)\end{array}$ & $\begin{array}{c}-0.315^{* *} \\
(0.127)\end{array}$ \\
\hline Highest Quintile & $\begin{array}{c}-1.051 * * * \\
(0.160)\end{array}$ & $\begin{array}{c}-1.077 * * * \\
(0.160)\end{array}$ & $\begin{array}{c}-0.509 * * * \\
(0.133)\end{array}$ \\
\hline Flood shock at Birth & $\begin{array}{c}3.990 * * * \\
(1.033)\end{array}$ & $\begin{array}{c}3.582 * * * \\
(1.066)\end{array}$ & $\begin{array}{c}2.580 * * * \\
(0.878)\end{array}$ \\
\hline Teenage Pregnancy & & & $\begin{array}{c}2.313 * * * \\
(0.0856) \\
\end{array}$ \\
\hline $\mathrm{FE}$ & no & yes & yes \\
\hline $\mathrm{R} 2$ & 0.271 & 0.274 & 0.508 \\
\hline $\mathrm{F}$ & 57.53 & 58.15 & 144.3 \\
\hline Observations & 1555 & 1555 & 1555 \\
\hline
\end{tabular}

Standard errors in parentheses

1999-2000 DHS Data. OLS regression; dependent variable is Child Marriage- the probability of marriage before age 18 .

$* \mathrm{p}<0.10, * * \mathrm{p}<0.05, * * * \mathrm{p}<0.010$ 
Table 1.9: IV Results - Choice of Instruments

\begin{tabular}{|c|c|c|c|}
\hline & $\begin{array}{l}\text { (1) } \\
\text { IV }\end{array}$ & $\begin{array}{l}\text { (2) } \\
\text { IV }\end{array}$ & $\begin{array}{l}\text { (3) } \\
\text { IV }\end{array}$ \\
\hline Child Marriage & $\begin{array}{l}0.0395 * * \\
(0.0201)\end{array}$ & $\begin{array}{c}0.0453 * * \\
(0.0192)\end{array}$ & $\begin{array}{l}0.0811 * \\
(0.0479)\end{array}$ \\
\hline Urban & $\begin{array}{l}-0.0125 \\
(0.0309)\end{array}$ & $\begin{array}{l}-0.0123 \\
(0.0302)\end{array}$ & $\begin{array}{l}-0.00881 \\
(0.0312)\end{array}$ \\
\hline Mother Primary Edu & $\begin{array}{l}-0.0213 \\
(0.0361)\end{array}$ & $\begin{array}{l}-0.00848 \\
(0.0354)\end{array}$ & $\begin{array}{l}-0.00343 \\
(0.0358)\end{array}$ \\
\hline Mother Literate & $\begin{array}{l}-0.0360 \\
(0.0373)\end{array}$ & $\begin{array}{l}-0.0312 \\
(0.0364)\end{array}$ & $\begin{array}{l}-0.0204 \\
(0.0408)\end{array}$ \\
\hline Father Primary Edu & $\begin{array}{c}-0.107 * * * \\
(0.0309)\end{array}$ & $\begin{array}{c}-0.106 * * * \\
(0.0302)\end{array}$ & $\begin{array}{c}-0.0970 * * * \\
(0.0331)\end{array}$ \\
\hline Lowest Quintile & $\begin{array}{c}0.0181 \\
(0.0413)\end{array}$ & $\begin{array}{l}0.00229 \\
(0.0405)\end{array}$ & $\begin{array}{l}0.00221 \\
(0.0412)\end{array}$ \\
\hline Second Quintile & $\begin{array}{l}-0.0305 \\
(0.0413)\end{array}$ & $\begin{array}{l}-0.0422 \\
(0.0405)\end{array}$ & $\begin{array}{l}-0.0405 \\
(0.0411)\end{array}$ \\
\hline Fourth Quintile & $\begin{array}{l}-0.0619 \\
(0.0431)\end{array}$ & $\begin{array}{l}-0.0705^{*} \\
(0.0423)\end{array}$ & $\begin{array}{c}-0.0709 * \\
(0.0429)\end{array}$ \\
\hline Highest Quintile & $\begin{array}{c}-0.144 * * * \\
(0.0487)\end{array}$ & $\begin{array}{c}-0.148 * * * \\
(0.0477)\end{array}$ & $\begin{array}{c}-0.145 * * * \\
(0.0496)\end{array}$ \\
\hline Flood shock at Birth & $\begin{array}{l}-0.497 * \\
(0.279)\end{array}$ & $\begin{array}{c}-0.584 * * \\
(0.282)\end{array}$ & $\begin{array}{c}-0.650 * * \\
(0.302)\end{array}$ \\
\hline Teenage Pregnancy & & & $\begin{array}{l}-0.146 \\
(0.122) \\
\end{array}$ \\
\hline $\begin{array}{l}\mathrm{FE} \\
\mathrm{R} 2\end{array}$ & $\begin{array}{c}\text { no } \\
0.0888\end{array}$ & \begin{tabular}{c}
\multicolumn{1}{c}{ yes } \\
00836
\end{tabular} & $\begin{array}{c}\text { yes } \\
00527\end{array}$ \\
\hline $\begin{array}{l}\text { Sargan p-value } \\
\text { Cragg-Donald F } \\
\text { Observations }\end{array}$ & $\begin{array}{l}170.6 \\
1555 \\
\end{array}$ & $\begin{array}{r}. \\
178.7 \\
1555 \\
\end{array}$ & $\begin{array}{r}. \\
43.79 \\
1555 \\
\end{array}$ \\
\hline
\end{tabular}

Standard errors in parentheses

1999-2000 DHS Data. Columns 1-3 use the average effect of flood shocks across ages 12 to 17 as instruments. The dependent variable is children's health outcomes, measured by stunting.

$* \mathrm{p}<0.10, * * \mathrm{p}<0.05, * * * \mathrm{p}<0.010$ 
Table 1.10: IV Results - Effect on Moderate Stunting and Severe Stunting

\begin{tabular}{|c|c|c|c|c|}
\hline & $\begin{array}{c}\text { (1) } \\
\text { Moderate Stunting }\end{array}$ & $\begin{array}{c}\text { (2) } \\
\text { Moderate Stunting }\end{array}$ & $\begin{array}{c}\text { (3) } \\
\text { Severe Stunting }\end{array}$ & $\begin{array}{c}\text { (4) } \\
\text { Severe Stunting }\end{array}$ \\
\hline Child Marriage & $\begin{array}{l}0.000785 \\
(0.0156)\end{array}$ & $\begin{array}{l}-0.00257 \\
(0.0449)\end{array}$ & $\begin{array}{c}0.0497 * * * \\
(0.0135)\end{array}$ & $\begin{array}{l}0.0820 * * \\
(0.0400)\end{array}$ \\
\hline Urban & $\begin{array}{l}-0.0163 \\
(0.0287)\end{array}$ & $\begin{array}{l}-0.0169 \\
(0.0292)\end{array}$ & $\begin{array}{l}0.00564 \\
(0.0249)\end{array}$ & $\begin{array}{l}0.00820 \\
(0.0261)\end{array}$ \\
\hline Mother Primary Edu & $\begin{array}{l}0.00225 \\
(0.0336)\end{array}$ & $\begin{array}{l}0.00255 \\
(0.0335)\end{array}$ & $\begin{array}{l}-0.00348 \\
(0.0292)\end{array}$ & $\begin{array}{l}0.00258 \\
(0.0299)\end{array}$ \\
\hline Mother Literate & $\begin{array}{l}0.00736 \\
(0.0340)\end{array}$ & $\begin{array}{l}0.00546 \\
(0.0382)\end{array}$ & $\begin{array}{l}-0.0343 \\
(0.0296)\end{array}$ & $\begin{array}{l}-0.0264 \\
(0.0341)\end{array}$ \\
\hline Father Primary Edu & $\begin{array}{c}-0.0716 * * \\
(0.0286)\end{array}$ & $\begin{array}{c}-0.0727 * * \\
(0.0310)\end{array}$ & $\begin{array}{l}-0.0315 \\
(0.0248)\end{array}$ & $\begin{array}{l}-0.0238 \\
(0.0276)\end{array}$ \\
\hline Lowest Quintile & $\begin{array}{l}-0.0572 \\
(0.0385)\end{array}$ & $\begin{array}{l}-0.0570 \\
(0.0385)\end{array}$ & $\begin{array}{l}0.0557^{*} \\
(0.0335)\end{array}$ & $\begin{array}{c}0.0559 \\
(0.0344)\end{array}$ \\
\hline Second Quintile & $\begin{array}{l}-0.0279 \\
(0.0385)\end{array}$ & $\begin{array}{l}-0.0276 \\
(0.0385)\end{array}$ & $\begin{array}{l}-0.0171 \\
(0.0335)\end{array}$ & $\begin{array}{l}-0.0148 \\
(0.0343)\end{array}$ \\
\hline Fourth Quintile & $\begin{array}{c}-0.0949 * * \\
(0.0395)\end{array}$ & $\begin{array}{c}-0.0968 * * \\
(0.0402)\end{array}$ & $\begin{array}{c}0.0271 \\
(0.0344)\end{array}$ & $\begin{array}{c}0.0231 \\
(0.0358)\end{array}$ \\
\hline Highest Quintile & $\begin{array}{c}-0.125^{* * *} \\
(0.0437)\end{array}$ & $\begin{array}{c}-0.128 * * * \\
(0.0464)\end{array}$ & $\begin{array}{l}-0.0210 \\
(0.0380)\end{array}$ & $\begin{array}{l}-0.0237 \\
(0.0413)\end{array}$ \\
\hline Flood Shock at Birth & $\begin{array}{c}0.00804 \\
(0.266)\end{array}$ & $\begin{array}{l}0.0177 \\
(0.283)\end{array}$ & $\begin{array}{c}-0.594 * * \\
(0.231)\end{array}$ & $\begin{array}{c}-0.646^{* *} \\
(0.252)\end{array}$ \\
\hline Teenage Pregnancy & & $\begin{array}{c}0.00457 \\
(0.115) \\
\end{array}$ & & $\begin{array}{l}-0.149 \\
(0.102) \\
\end{array}$ \\
\hline $\mathrm{FE}$ & yes & yes & yes & yes \\
\hline $\mathrm{R} 2$ & 0.0224 & 0.0218 & 0.0210 & -0.0298 \\
\hline Sargan p-value & 0.805 & 0.783 & 0.536 & 0.0717 \\
\hline Cragg-Donald F & 128.8 & 21.90 & 128.8 & 21.90 \\
\hline Observations & 1555 & 1555 & 1555 & 1555 \\
\hline
\end{tabular}

Standard errors in parentheses

1999-2000 DHS Data. Columns 1 to 4 use the average effect of drought and flood shocks across ages 12 to 17 as instruments. The dependent variable is children's health outcomes, measured by moderate stunting (Columns 1 to 2 ) and severe stunting (Columns 3 to 4 ).

$* \mathrm{p}<0.10, * * \mathrm{p}<0.05, * * * \mathrm{p}<0.010$ 


\section{CHAPTER 2}

\section{CHILD MARRIAGE AND MORTALITY OUTCOMES OF OFFSPRING}

\subsection{Introduction}

Child mortality is an important determinant of development of a country. Child mortality rates have decreased globally in the past 25 years, falling from 91 deaths per 1000 live births in 1990 to 43 deaths in 2015. Despite progress, child mortality rates in South Asia and Sub-Saharan Africa are still very high, and account for more than 80 percent of global under-five deaths. In particular, Bangladesh continues to be one of the top 10 countries with the highest number of under-five deaths; the United Nations Inter-Agency Group for Child Mortality Estimation reports that 119,000 children die before reaching the age of five (UNICEF 2015). Of related concern is the prevalence of Child Marriage within the country. Bangladesh has the fourth highest number of Child Marriages worldwide, with approximately 52 percent of women being married before the age of 18 , and 18 percent being married before the age of 15 (UNICEF 2016).

Child Marriage is associated with a range of negative consequences for the child bride, including lower rates of educational attainment, increased vulnerability to intimate partner violence and lower status and decision-making autonomy within the groom's household. The negative consequences rendered are channels through which Child Marriage influences the outcomes of offspring. Consider the child bride's lower decision-making autonomy in the household; the inability of the child bride to advocate for herself and to make decisions on her own health care and children's health care affects the welfare of her 
children. While the situation of child brides has been extensively documented (Parsons et.al 2015, Jensen and Thornton 2003, Malhotra 2013), evidence on the ways in which Child Marriage may limit and compromise the outcomes of the children produced from these unions is limited. Specifically, little is known about whether mortality outcomes of children differ between women who marry early, and those who marry later. Only two previous studies have investigated the relationship between Child Marriage and children's mortality outcomes. Guilbert (2013) uses a probit estimation and finds that early marriage increases the likelihood of child death and the average number of dead children per woman in Senegal. On the other hand, Raj et al. (2010) uses cross-sectional analyses and shows no significant associations between Child Marriage and infant and child mortality. Understanding the relationship between Child Marriage and child mortality is important to identify whether Child Marriage has an intergenerational impact. Such an assessment is central in designing effective policies to promote child survival and to justify ending Child Marriage.

This paper aims to address the gap in the literature by investigating the effect of Child Marriage on mortality outcomes using child mortality ${ }^{9}$ and infant mortality ${ }^{10}$ as measures of outcomes. The main empirical challenge in establishing a causal link is the endogeneity that can arise and bias the association between Child Marriage and the mortality outcomes of children, thereby leading to an underestimation of the true impact. To illustrate, the woman's natal family may place less interest in the welfare of their children and lower

\footnotetext{
${ }^{9}$ Child mortality refers to the death of children before the age of 5 .

${ }^{10}$ Infant mortality measures the death of infants before the age of 1.
} 
their investment in them. This may lead to the natal family marrying their daughters earlier. These viewpoints may shape the behavior of the woman in childhood and are liable to be replicated in her later adult life, thus leading to women taking care of their children in a less attentive manner. Consider also if the woman's natal family follows traditional norms and believes in alternative medicine, thereby providing less access to health care services (immunizations, vitamin A supplementation). These preferences in traditional practices may lead to the household being more supportive of Child Marriage. Additionally, if women reproduce these preferences, they can affect their children's wellbeing through lower use of Western medicinal treatment from health care facilities.

To solve the endogeneity problem, this paper develops and implements an instrumental variable strategy to study the causal effect of Child Marriage on mortality outcomes. In particular, I exploit the exogenous variation in rainfall shocks across clusters in Bangladesh to use flood shocks as an instrumental variable for Child Marriage ${ }^{11}$. In Bangladesh, the agricultural sector is an important component of the economy. In the time period of data collection, the agricultural sector employed 64 percent of the labor force and contributed to over 30 percent of the GDP. Agricultural productivity is strongly dependent on weather patterns, and fluctuations in rainfall result in a large variation in agricultural yields, and consequently household income. Using the Standard Precipitation Index, I construct the pattern of moderate flood shocks each mother experienced from the ages of 12 to 17 to examine the relationship between flood shocks and the timing of marriage. I specifically choose to examine moderate flood shocks due to the positive effect of moderate

\footnotetext{
${ }^{11}$ In the first chapter, I develop a theoretical model that illustrates the relationship between Child Marriage and income shocks.
} 
floods on rice yields, and thus the positive effect on household income. In Bangladesh, rice is the principal agricultural crop and floods—as long as they are moderate episodes—are beneficial to rice yields (Brebbia 2013, Nakao 1996). When a household experiences a moderate flood shock in a particular year, the shock can impact the parents' decision to marry their daughter in that year. If the moderate flood shock increases crop yields and household income, the economic burden of providing for the daughter in the household is alleviated. The economic circumstances of the household play a central role in the decision to marry early; Child Marriage is often used as a tool to lessen costs (Nour 2006). Previous studies have examined the relationship between rainfall shocks and the age at marriage ${ }^{12}$; Corno and Voena (2015) use data from Tanzania to show that adverse rainfall shocks increase the probability of marriage by age 18; Corno, Hildebrandt and Voena (2016) find differing effects from the impact of drought and flood shocks on the hazard into early marriage in Africa and India.

Using information from the 1999-2000 Bangladesh Demographic Health Surveys (BDHS) and historical rainfall data from the Terrestrial Air Temperature and Precipitation dataset (Matsuura and Willmott 2007), I examine the relationship between Child Marriage and offspring's mortality outcomes. In addition, I explore the channels connecting Child Marriage to mortality outcomes by examining the effect of Child Marriage on a woman's status in the household, using her decision-making ability as an agency of empowerment.

The results I obtain indicate that Child Marriage substantially worsens the outcomes of children. In comparison to children produced from later-married mothers, children from

\footnotetext{
${ }^{12}$ In the first chapter, I use rainfall shocks to analyze the effect of Child Marriage on the health outcomes of firstborn children.
} 
Child Marriage unions are 67.9 percentage points more likely to die before reaching the age of five. The effect on infant mortality is smaller; children are 24.8 percentage points more likely to die before reaching the age of one year. By examining the effect by gender, the results suggest that there is clear differential impact on mortality outcomes. Male children from Child Marriage unions face a higher probability of child mortality, while the effect on female children is smaller. Child Marriage leads to an increase in likelihood of male infant mortality. However, I find no evidence of the effect on female infant mortality. These results imply that the predominant effect of Child Marriage is concentrated through increases in male child and infant mortality.

The evidence I find on the relationship between Child Marriage and the status of women in the household indicates that Child Marriage significantly lowers women's decision-making autonomy and access to health care. In comparison to later-married women, women who are married before the age of 18 are more likely to have problems in gaining permission to go to health facilities and are less able to make health care decisions for themselves and for their children.

The remainder of the paper is organized as follows. Section 2.2 describes the channels of impact of Child Marriage on children's outcomes. Section 2.3 details the data used and provides some descriptive statistics. The empirical strategy is discussed in Section 2.4. Section 2.5 provides the main empirical results, while Section 2.6 provides a supplementary analysis. Section 2.7 concludes the paper. 


\subsection{Channels of Impact of Child Marriage on Offspring's Mortality}

In this section, I discuss the intergenerational impact of Child Marriage by documenting the channels through which Child Marriage affects the health outcomes of offspring. The three channels I explore are: lower educational attainment, increased vulnerability to violence and reduced decision-making and autonomy in the household ${ }^{13}$.

Upon marriage, child brides are prevented from continuing their education (Chowdhury 2014, Vogelstein 2013, Lloyd and Mensch 2008). They are expected to devote their time solely to domestic, marital and child-bearing duties (Nour 2006). Education and marriage are considered to be incompatible; Field and Ambrus (2008) find that a delay in Child Marriage by one year between ages 11 and 16 results in an increase in schooling by an average of 0.22 year and an increase in literacy by 4.1 percentage points; Nguyen and Wodon (2012) show an additional year of Child Marriage is associated with a 5.7 percentage points decrease in literacy and a reduction in secondary schooling by 5.6 percentage points. The impact of the loss in educational attainment is twofold-first, it severely limits the child bride's future employment opportunities and earnings potential. Second, it serves to isolate the child bride by depriving her of an environment beyond the household where she can interact with peers of her own age, develop social skills and build a support network (Lee-Rife et al. 2012). The lower level of education combined with restricted access outside of the household affects children's health outcomes through the inability of the child bride to access knowledge on child care and family planning (Parsons et al. 2015). Smith and Haddard (2014) provide confirmation of women's education as a

\footnotetext{
${ }^{13}$ Health risks related to early pregnancy and childbearing are not applicable as a channel affecting children as my analysis only examines children's cases from non-adolescent mothers
} 
key determinant of reduction in child undernutrition. Similarly, Ruel et al. (2013) highlight that the educational attainment of women has a positive impact on the quality of care their children receive, shown by the duration of breast-feeding and health care seeking during illnesses.

While one of the reasons parents opt for Child Marriage is to safeguard their daughters against violence and unwanted sexual advances from men in conflict-prone regions (Loaiza and Wong 2012), child brides face a higher susceptibility to intimate partner violence than same-age peers who marry later (Santhya et al., 2007, Black 2001, Raj 2010). Intimate partner violence can be characterized as physical, sexual and mental. Physical violence includes the use of physical force and forms of torture and is exhibited through hitting, slapping, whipping and kicking. Sexual violence is sexual activity that is imposed by threats, intimidation or any form of pressure. Mental violence comprises of harmful interactions intended to scar, terrorize, humiliate and threaten. It includes insults, name calling, bullying, isolating and neglecting needs (UNICEF 2014). Jensen and Thornton (2003) indicate that there is a substantial difference in the rate of reported violence (physical, mental and sexual) between women that marry early and later-married women. Similarly, using data from Ethiopia, Erulkar (2013) finds a strong association between Child Marriage and sexual violence. Compared to brides married at 18 to 19 , child brides are four times more likely to experience forced first sex with their spouse. Comparably, in an analysis using data from Bangladesh, Rahman et al. (2014) show an increased risk of physical intimate partner violence for child brides that continues into their subsequent family life. 
Being subjected to intimate partner violence can affect the children of child brides even before they are born. Violence experienced during pregnancy can induce the stress hormone cortisol to increase, resulting in poor fetal growth and brain developmental issues (Mulder et al 2002, Quinlivan 2000). In addition, the experience of violence impacts the mental health of child brides by putting them at a higher risk for stress, anxiety, depression, suicide and post-traumatic disorders (Raj 2010, Nour 2009, Carbone-Lopez et al. 2006). Children are liable to be affected, owing to the failure of mothers to obtain adequate nutrition and medical care during pregnancy and after they are born, as a result of the negative impact on their mental health. In a study by Kabeta (2010) analyzing the effect of maternal depression and violence on child survival, results show a higher risk of child death from mothers who experienced episodes of depression and mental and physical violence. In addition, children are sensitive to their environment and prolonged exposure to violence in early childhood can affect their physical health by increasing the risk for developmental delays (UNICEF 2014)

One of the reasons grooms choose child brides is to establish control, thwart opportunities that would allow them to gain awareness of their rights and stop them from developing an independent or self-reliant personality. Aiding in this is the exclusion of child brides from school, which limits their chances of acquiring skills and accessing better-paid work opportunities (Loaiza and Wong 2012). Confinement is further heightened by the restrictions placed on visiting their natal family and friends (Nour 2009). This all serves to disempower the child bride by increasing her economic dependency on the groom and placing her in a position where she is unable to advocate for herself. As a result, women from Child Marriage unions have less agency in societal and household 
decision-making (Jensen and Thornton 2003, Parsons et al. 2015, Guilbert 2013). Whether - and how much — a child bride has a voice in household decision-making can impact the welfare of her children. Smith et al. (2005) provide evidence confirming that improvements in women's decision-making power have a significant positive effect on the nutritional status of their children. Maitra (2004) examines the relationship between the status of a woman in the household and health care decisions, and shows that a woman's control over household resources has a significant positive effect on the demand for prenatal care and the probability of birth in a hospital. Women with greater status in the household have more control over resources, possess more self-confidence and selfesteem, take better care of themselves, have higher nutritional status and are able to provide higher-quality care to their children. Numerous studies report the importance of women's involvement in household decision-making on the reduction in child-mortality and child malnutrition (Fantahun et al. 2007, Shroff et al 2009, Dancer and Rammohan 2009). To illustrate, Fantahun et al. (2007) show that the mortality of children is three times higher in households where women have low decision-making power in comparison to households where they have high decision-making power. Likewise, Hossain et al. find an adverse association between children's survival and the constrained authority and autonomy of women in Bangladesh.

In section 2.6, I contribute to this literature by examining the effect of Child Marriage on the status of women in the household, using their decision-making ability as a measure of agency and empowerment. 


\subsection{Data Section}

\subsubsection{Child Outcomes Data}

To examine the effect of Child Marriage on child and infant mortality, I use micro data from the 1999-2000 Bangladesh Demographic and Health Survey (BDHS). The survey was conducted by researchers from the firm Mitra and Associates under authorization from the National Institute of Population Research and Training (NIPORT). The survey provides comprehensive information on maternal and child health, family planning and the nutritional status of mothers and children. The survey is based on a nationally representative sample of ever-married women aged from 10 to 49 from 10,268 households. A total of 341 primary sampling units (clusters) are used in the survey, and the geographic coordinates of each cluster are provided. The GPS information is necessary to implement the instrumental variables strategy, where it is used to link clusters to weather grid cells.

Women in the survey are asked to provide a history of all births. For each birth, information on the year of birth, sex, and age at death if the child is dead at the time of interview, is collected. To allow for child mortality exposure, I exclude children that were born in the five years preceding the survey. Child mortality is defined as the death of children under the age of five. The inclusion of all births will lead to incomplete information on the survival status of children that were born in the last five years before the survey. The missing data can potentially underrepresent the incidence of child mortality and produce misleading results by introducing bias. Similarly, when the dependent variable is infant mortality, I drop all children that were born in the year preceding the survey. In 
addition, I limit the analysis to children whose mothers were aged over 18 at their birth ${ }^{14}$. This covers 80 percent of children in the sample for child mortality and 81 percent in the sample for infant mortality. The final sample used in the child mortality analysis consists of 20,919 cases, and the sample used in investigating the effect of Child Marriage on infant mortality contains 25,511 cases.

A key advantage of the BDHS dataset is the ability to link the children's birth records to data from their mothers. The main independent variable, Child Marriage, is constructed from the question regarding the age at marriage. If the age at marriage of the mother is reported as below 18, then these children are identified as the offspring of Child Marriage unions.

Table 2.1 and Table 2.2 present the summary statistics of selected characteristics for mothers, households and children in the samples used for child mortality and infant mortality analysis, respectively. The mean age of marriage for mothers is low at 14 years. Approximately 90 percent of the children are from Child Marriage unions. In the child mortality sample, approximately 58 percent are born to mothers who were married before the age of 15 , while in the infant mortality sample, 56 percent are from mothers married before 15. Birth characteristics show that the mean age at birth occurs at 24 years and approximately 10 percent of children were born within 18 months of the previous birth. The number of surviving children in the household at the time of the birth of the child is approximately three children.

\footnotetext{
${ }^{14}$ Child Marriage is associated with early pregnancy. Due to the health risks related to early pregnancy, I exclude children who were born before their mother was 18 in order to comparatively assess the outcomes of children from Child Marriage to children from non-Child Marriage unions.
} 
From looking at the educational characteristics of the parents, fathers possess a higher level of education (primary school or higher) than mothers. The children are largely from a nuclear family structure (approximately 79 percent) and are in a rural location. In reference to the wealth levels, approximately 38 percent of children are from households below the middle quintile (lowest and second quintile), and 43 percent are from households above the middle quintile (fourth and highest quintile). The number of firstborn children in each sample is approximately 17 percent. The samples are nearly evenly split, with the number of male children exceeding females by 2 percent. The mortality patterns from each sample show that 14 percent of children die before the age of five and approximately 9 percent of children die before the age of one.

Figure 2.1 displays the incidence of male and female child mortality. The pie charts show that there is no substantial difference between the deaths of male and female children before the age of five. Out of the male children born, 14.02 percent die before reaching the age of five, and of all female children born, 14.53 percent die. However, there is a noticeable difference in mortality outcomes for male and female children before the age of one, as displayed in Figure 2.2. The pie charts show that of all male children born, approximately 9.90 percent die before the age of one. For female children, the incidence of infant mortality is lower; the data show that 8.96 percent die.

In consideration of differences in mortality by birth order, Figure 2.3 and Figure 2.4 display the occurrence of child mortality and infant mortality in firstborn and later-born children. For child mortality, the pie charts for firstborn and later-born children are fairly similar; the data show that 14.53 percent of firstborn children die before reaching five years of age and 14.22 percent of later-born children die. In contrast, the pie charts for infant 
mortality show a difference between firstborn and later-born children. Out of all firstborn children, 11.35 percent die before reaching one. The infant mortality outcomes for laterborn children are comparatively lower at approximately 9.04 percent.

\subsubsection{Rainfall Data}

I obtain historical rainfall data for weather stations across Bangladesh from the University of Delaware dataset (Matsuura and Willmott 2007). Precipitation from 1900 to 2010 is recorded monthly on a 0.5 degree latitude by 0.5 degree longitude global grid. As the area of interest lies in the probability of Child Marriage stemming from income shocks created by rainfall shocks, and in consideration of the age of the mothers in the sample, I specifically use rainfall data from 1940 onwards and match each cluster in the BDHS dataset to the closest weather grid cell.

To characterize flood shocks, I construct a Standardized Precipitation Index (SPI). The SPI developed by McKee et al. $(1993,1995)$ serves as one of the best indicators to detect and monitor the frequency of flood events by studying the departures from normality (Guerreiro et al.2008). Theoretically, the SPI is defined as the difference in precipitation from the long-term mean for a given time scale, divided by the standard deviation (Dobos 2013, Giddings et al. 2005, Paltineanu et al. 2009):

$S P I_{y, c}=\frac{P_{y, c}-\overline{\bar{P}}_{c}}{\sigma_{c}}$

where $P_{y, c}$ is the observed precipitation in year y at in cluster $c . \overline{\bar{P}}_{y, s}$ is the long-term mean (1940-2010) in each cluster and $\sigma_{c}$ is the standard deviation. 
Flood shocks are constructed for every year that the mother is at ages $12,13,14,15$, 16 and 17. I use a binary indicator for whether a year constitutes a flood shock, and define a flood shock as an SPI value ranging from 1 to 2 :

Floodshock $_{y, c}=1\left\{1 \leq S P I_{y, c} \leq 2\right\}$

The primary advantage of the SPI is the ability to assess the severity of flood events. By using values of the SPI below the threshold of 2, moderate flood shocks can be clearly identified $^{15}$. This differentiation is pertinent to establish flood shocks as positive income shocks in the analysis. Flood shocks have previously been used as a proxy for negative income shocks due to the damaging effect of floods on crop yields (Asada et al. 2005). However, most of these studies have either investigated the effect of severe (extreme) flood events (Rosales-Rueda 2014, Ferreira and Ghimire 2012) or highlighted the negative effects on agricultural activity by focusing only on crop damage data (Asada et al. 2005, Murshid 1987, Brammer 1990 and Paul and Rasid 1993). In contrast, the literature that takes into account differences in the severity of floods finds that moderate floods have positive implications for crop yields and agricultural productivity (Cunado and Ferreira 2014; Fomby et al. 2013).

Bangladesh currently ranks as the fourth country in the world in rice production (FAO 2016). Rice is the principal agricultural crop. Rice accounts for 90 percent of total grain production and occupies 80 percent of agricultural land (Asaduzzaman et al., 2012). Numerous studies have documented the strong association between fluctuations in rice production and annual rainfall variations (Mowla 1976, Asada and Matsumoto 2009).

\footnotetext{
${ }^{15}$ SPI values from 1 to 2 are representative of moderately wet and very wet episodes. SPI values greater than 2 are associated with extremely wet periods (McKee et al. 1993, Guerreiro et al. 2008)
} 
Floods, as long as they are moderate in degree, are beneficial to rice yields as they bring water and naturally fertilize the soil by depositing nutrients and sediments over rice plains (Nakao 1996, WMO 2006).

\subsection{Empirical Strategy}

To examine the relationship between Child Marriage and the mortality outcomes of offspring, I estimate the following linear probability model:

$Y_{i, c}=\alpha_{0}+\alpha_{1}$ ChildMarriage $_{i, c}+\alpha_{2} X_{i, c}+\gamma_{t}+\delta_{c}+\mu_{i, t, c}$

where $Y_{i, c}$ is the mortality outcome of child $i$ in cluster $c$. Outcome variables are dichotomous and include child mortality (death of the child before the age of five years) and infant mortality (death of the child before one year). ChildMarriage $e_{i, c}$ is a binary indicator for whether the mother of child $i$ was married before the age of 18. The vector $X_{i, c}$ is a set of individual- and household-level controls. These cover the mother's age at birth, household wealth levels, educational levels of the mother and father, household location (urban or rural), household family structure (nuclear or extended), living children in the household at the time of birth and birth interval.

These controls are specifically chosen in light of the evidence that identifies certain socioeconomic and demographic factors as determinants of child and infant mortality (Kim 1988, Das Gupta 1990, Mosley and Chen 1984, Gubhaju, Streatfield and Majumder 1991). To illustrate, Kabir et al. (2001) find that the socioeconomic status of parents in relation to maternal education and access to water ${ }^{16}$ reduces the risk of child and infant mortality.

\footnotetext{
${ }^{16}$ The household's access to water is incorporated in the calculation of the wealth index. The wealth index is a cumulative measure of the household's living standard. It is constructed from data on the household's
} 
Further, Majumder et al. (1997) identify parental education and demographic factors such as birth interval and survival of preceding child (children) as key determinants. Similarly, Cleland and Sathar (1984) and Pebley and Millman (1986) both document the importance of one particular demographic factor: birth interval length.

Dummy variables are constructed and assigned a value of 1 if each of the following holds true: the household is in an urban location, the child is from a nuclear family and the interval between births is within 18 months. Otherwise, the variable is assigned a value of 0 . The controls for mother's age at birth and living children in the household at the time of birth are both continuous variables. Household wealth levels are measured from the wealth index by four dummy variables: lowest quintile, second quintile, fourth quintile and highest quintile. The lowest quintile is representative of the poorest 20 percent of households, while the highest quintile corresponds to the richest 20 percent of households in Bangladesh. The educational levels of the mother and father are each denoted by a dummy variable that is equal to 1 if the highest level of education achieved is primary school or higher, and 0 otherwise. Cluster fixed effects $\delta_{c}$ and year of birth $\gamma_{t}$ fixed effects are included to control for unobserved heterogeneity in the cluster and in the year the child was born.

I estimate Equation (2.1) using OLS. A central challenge with the OLS approach is the endogeneity which may arise and bias the association between Child Marriage and the mortality outcomes of children. This may be due to unobserved confounding factors that affect both the marriage age and the mortality outcomes of offspring. For example, the woman's natal family may place less value on children. This behavior leads to parents

assets, household materials used in construction, and the types of water access and sanitation facilities (BDHS) 
supporting an earlier marriage, and if replicated leads to women taking care of their children in a less attentive fashion. Consider also if the woman's natal family follows traditional norms and believes in alternative medicine, thus providing less access to health care services (e.g., immunizations, vitamin A supplementation). These preferences lead to the involvement of the natal family in early marriages for their daughters. These preferences can shape the behavior of the woman in childhood, and lead women to reproduce these behaviors in their later life, whereby they affect their children's wellbeing through lower use of Western medicinal treatment from health care facilities.

As a solution to the endogeneity problem, I employ an instrumental variables approach. I exploit the exogenous variation in rainfall shocks across clusters in Bangladesh as an instrumental variable for Child Marriage. The idea behind this instrument is based on the following: the household's decision to marry their daughter is influenced by the variation in household income. Fluctuations in rainfall are an important source of variation in income owing largely to the household's dependency on the agricultural sector. In Bangladesh, the agricultural sector is central to its economy; it employs 47.3 percent of the labor force and contributes 16.33 percent of the GDP (Bank 2014). In the time period preceding the survey, the agricultural sector accounted for 64 percent of the labor force and contributed 30 percent to GDP. Agricultural productivity is strongly dependent on weather patterns, and variations in rainfall can result in large variations in agricultural output and thus household income. Previous works have examined the relationship between rainfall shocks and the age at marriage. Corno and Voena (2015) use data from Tanzania to show that adverse rainfall shocks increase the probability of marriage by age 18. Corno, Hildebrandt and Voena (2016) find that the impact of rainfall shocks on the hazard into 
early marriage differs; droughts increase the early marriage hazard in Africa, while in India, drought decreases the hazard.

I estimate the following two stage model to study the causal effect of Child Marriage on the mortality outcomes of children:

$Y_{i, c}=\alpha_{0}+\alpha_{1}$ ChildMarriage $_{i, c}+\alpha_{2} X_{i, c}+\gamma_{t}+\delta_{c}+\mu_{i, t, c}$

Child Marriage $_{i, c}=\alpha_{0}+\alpha_{1}$ FloodShockAtAgea $_{i, c}+\alpha_{2} X_{i, c}+\gamma_{t}+\delta_{c}+\mu_{i, t, c}$

where FloodShockAtAgea $a_{i, c}$ is the measure of rainfall shocks that is used as an instrument to identify the first-stage equation. FloodShocka $a_{i, c}$ is an indicator for whether the mother of child $i$ experienced a flood shock at age $a$ in cluster $c$. For example, FloodShockAtAge $14_{i, c}$ takes on a value of 1 if the mother of child $i$ experienced a flood shock at age 14, and 0 otherwise. As the topic of concern is Child Marriage, only ages (a) from 12 to 17 are used in the analysis. Girls are withheld from the marriage market until the onset of puberty (Field and Ambrus 2008). The minimum age is assumed to be 12 as the average onset of puberty for girls in Bangladesh occurs at 12 years. The coefficient of interest is $\alpha_{1}$, the impact of instrumented Child Marriage on the mortality outcomes of children. The vector, $\mathrm{X}_{\mathrm{i}, \mathrm{c}}$ includes the same controls described earlier.

The mortality outcomes of children produced from Child Marriage unions are likely to vary by sex. There is no consensus in the literature on gender differentials in childhood mortality. While there is a higher biological risk of death among male children than among female children (Van Poppel 1979), son preference behavior is widespread in the South Asian region (Arnold et al.1997). Parents with strong son preference may discriminate against daughters by providing inferior care in terms of food distribution, medical care and 
access to education (Chen et al. 1981, Muhuri and Preston 1981, D’Souza and Chen 1980). To look at how the effect of Child Marriage on child mortality and infant mortality differs by sex, I estimate Equation (2.1) and Equation (2.2) separately for male and female children.

\subsection{Results}

\subsubsection{OLS Results}

Results from the Ordinary Least Squares estimation for the effect of child mortality on mortality outcomes are reported in Table 2.3. The dependent variable is child mortality in columns 1 to 2 and infant mortality in columns 3 to 4 , without and with fixed effects.

The results indicate that children from Child Marriage unions face a higher probability of dying. The coefficients on Child Marriage in all four regressions are statistically significant at the 1 percent level. The estimates suggest that the effect of Child Marriage is larger for child mortality than for infant mortality. With fixed effects, Child Marriage increases the likelihood of child mortality by 3.67 percentage points and infant mortality by 2.36 percentage points. The coefficients on the highest quintile, mother's primary education and living children in the household are negatively and statistically correlated with the probability of child and infant mortality, while the coefficients on the second quintile and birth interval are positively and statistically correlated. This direction of the correlations is in line with the literature (Fotso et al. 2013, Kabir et al. 2001, Das Gupta 1990); mother's education significantly influences child survival through improved child care; a birth interval of less than 18 months is associated with an increase in mortality 
risk $^{17}$; and households with better access to water and sanitary facilities (highest quintile) have a lower risk of mortality than those with less access to those facilities (second quintile and lowest quintile).

\subsubsection{Results}

\subsubsection{First-stage Regression Results}

For rainfall shocks to serve as a valid instrument for Child Marriage, the instrument must be sufficiently correlated with the endogenous explanatory variable.

First-stage regressions predicting the probability of Child Marriage in the child and infant mortality sample are reported in Table 2.4 and Table 2.5 , respectively. The results are presented with and without fixed effects and two sets of instruments are used. The first estimates the average effect of flood shocks across ages 12 to 17 on Child Marriage. However, the effect of flood shocks is likely to vary by age. Thus, to examine the distinct effects, the second set of instruments estimates the average effects of flood shocks across ages 12 to 13,14 to 15 and 16 to 17 .

The results from the first-stage regressions indicate that there is a negative and statistically significant relationship between each of the instruments and Child Marriage. In the child mortality sample (Table 2.4), if a woman experiences a flood shock between the ages of 12 and 17, the probability of marrying before 18 declines by 9.40 percentage points, with an F-statistic of 149 (column 4). The effect of flood shocks across different

\footnotetext{
${ }^{17}$ According to the maternal depletion theory, closely spaced births within 18 months can lead to pregnancy complications and low-weight babies, thus increasing the risk for child mortality (Majumder 1997).
} 
age intervals yields an F-statistic of 127.9 and shows that the largest decline in the probability of Child Marriage is from flood shock occurring between the ages 16 and 17 (column 2). The coefficients on the flood shocks in the infant mortality sample (Table 2.5) are not substantially different from the corresponding coefficients in Table 2.4.

Thus, the strong correlation between flood shocks and Child Marriage fulfills the requirement for identification in the IV model.

\subsubsection{Child Mortality and Infant Mortality Outcomes}

Table 2.6 and Table 2.7 present the IV results for the effect on child and infant mortality outcomes respectively, with and without year of birth and region fixed effects. Two sets of instruments are employed: flood shocks across age ranges 12 to 13,14 to 15 , 16 to 17 in columns 1 to 2 and the average of flood shocks across ages 12 to 17 in columns 3 to 4 . Standard errors are presented in parentheses, and the number of observations, Sargan p- value and Cragg Donald F-statistic are located in the last three rows of the table.

Focusing the analysis on child mortality, I find that Child Marriage has a substantial impact. Using flood shocks across different age intervals as instruments, the estimate shows that Child Marriage leads to an increase in child mortality ${ }^{18}$ by 67.9 percentage points $^{19}$. The estimated magnitude using the average of flood shocks as an instrument is similar and the coefficients on Child Marriage in both specifications are statistically different from zero at the 1 percent level. The results are robust to the inclusion of controls.

\footnotetext{
18 The increase of 0.679 points in child mortality from Child Marriage covers children who were born between 1968 and 1995.

${ }^{19}$ In terms of magnitude, an increase in one standard deviation in Child Marriage increases the probability of child mortality by 0.22 percentage points.
} 
Similar to the results in the OLS regression, the coefficients on the lowest quintile, second quintile and birth length, and the number of living children in the household are all statistically significantly and correlated.

Corresponding results for the effect on infant mortality are presented in columns 2 and 4 of Table 2.7. From using the average of flood shocks across different age intervals as instruments and applying fixed effects, the results indicate that Child Marriage increases the probability of children dying before 1 by 24.8 percentage points ${ }^{20}$. This effect is statistically significant at the 5 percent level. The coefficient on Child Marriage using the average of flood shocks as an instrument (column 4) is roughly the same size in magnitude but is statistically significant only at the 10 percent level.

The regression results thus far show that the effect of Child Marriage is larger for child mortality than infant mortality. The likelihood of children from Child Marriage unions dying before the age of five is approximately 2.7 times higher than the probability of dying before the age of 1 .

While the IV results and OLS results show very similar patterns, the magnitude of the coefficients in the IV specifications is larger. A potential explanation for the difference is the presence of omitted variable bias which induces endogeneity and leads to an underestimation of the true impact of Child Marriage on child mortality. Another possible explanation is that the attenuation bias caused by measurement $\operatorname{error}^{21}$ of Child Marriage reduces OLS coefficient estimates.

\footnotetext{
${ }^{20}$ On average, an increase in one standard deviation in Child Marriage is associated with a 0.08 percentage points increase in infant mortality.

${ }^{21}$ The age at marriage is reported by mothers in the survey. Measurement errors may be due to recall bias or misreporting of the age at marriage.
} 
I conduct diagnostic tests to assess the validity of the instruments and to evaluate how strong the identification is in the sample for child mortality and infant mortality. The model is overidentified in specifications using the average of flood shocks across different age intervals as instruments (columns 1 and 2 in Tables 2.6 and 2.7). An important assumption of the IV approach is that the excluded instruments are distributed independently of the error process. I use the Sargan statistic as a test of exogeneity and find that the Sargan pvalue fails to reject the null hypothesis that the overidentifying assumptions are valid. The high $\mathrm{p}$-values lend confidence to the validity of these instruments.

The Cragg-Donald F statistic is utilized to test whether the instruments are weak. I set the maximum acceptable bias to 0.10 ; tolerating a bias of 10 percent relative to OLS. In the child mortality sample, the size distortion is at less than 10 percent in the specification using the average of flood shocks as an instrument (column 4 in Table 2.6). The maximal size is less than 15 percent in the specification using multiple instruments. The F statistic (13.45) is larger than the critical value at 10 percent (9.08), indicating that the bias is small relative to OLS (column 2 in Table 2.6).

Correspondingly, in the infant mortality sample, the maximum size is less than 10 percent in the specification using the average of flood shocks as an instrument (column 4 in Table 2.7). In the specification with multiple instruments, the maximum size is less than 15 percent (column 2 in Table 2.7). The F statistic (20.554) is larger than the critical value at 5 percent (13.91). Accordingly, the Cragg-Donald F statistic shows that the set of instruments used in the regression analysis is strong. The relative bias does not exceed 10 percent and the maximum size distortion is no more than 15 percent, suggesting that the instruments cannot be deemed as weak. 


\subsubsection{Child and Infant Mortality Outcomes by Gender}

To explore the effect of Child Marriage on gender differences in child and infant mortality, I estimate Equation (2.2) separately for male and female children. IV results for the effect of Child Marriage on male child mortality and female child mortality are reported in Table 2.8. I use flood shocks across the ages 12 to 13,14 to 15 and 16 to 17 as instruments ${ }^{22}$ for Child Marriage. Columns 1 to 2 present the results without and with fixed effects for males, and columns 3 to 4 report the results for females.

The results indicate that both male and female children from Child Marriage unions have a higher likelihood of dying before the age of five. The coefficients on Child Marriage across male and female regressions are all statistically and significantly different from zero. The estimates also suggest that there is a clear differential impact on child mortality outcomes; the likelihood of male children increases by 89 percentage points if they result from Child Marriage unions. The result for female children is 62.1 percentage points, thus smaller by 26.1 percentage points.

The IV results for male and female infant mortality outcomes are presented in Table 2.9 $9^{23}$. In stark contrast, Child Marriage does not seem to influence the likelihood of infant mortality for female children. The coefficients on Child Marriage in the female infant mortality regressions (column 3 and 4 ) are smaller in magnitude than the corresponding coefficients in the male regressions (column 1 and 2) and none of the coefficients are statistically significant.

\footnotetext{
${ }^{22}$ First-stage results are presented in Table A3.

${ }^{23}$ First-stage results are presented in Table A4.
} 
The results thus far indicate that Child Marriage has a greater effect on the mortality outcomes for male children than for female children. The estimates imply that the predominant effect of Child Marriage is concentrated on increases in male child mortality and male infant mortality. While I find an effect on female child mortality, there is no indication that female deaths before the age of one are influenced by Child Marriage. The gender differences in mortality outcomes is consistent with evidence that shows that the chance of survival for male infants is lower than for female infants (Drevenstedt et al. 2008, Naeye et al. 1971, Waldron 1998). The lower survival rate for males can be attributed to gender differences in genetic and biological factors (Pongou 2013). Males are biologically weaker; Waldron (1983) documents that the presence of XY chromosomes in males makes them more vulnerable to recessive disorders than females with $\mathrm{XY}$ chromosomes. In addition, female infants have a stronger immune response than males, making them less susceptible to infectious and noninfectious diseases (Read et al. 1997 and Chao 1996, Ahmed et al.1985). As male children are more vulnerable, the inability of mothers that were married early to advocate on behalf of their own children has a stronger effect on them than on their female children. To illustrate, Child Marriage is associated with adverse physical and mental health consequences. The lack of access to health facilities in the prenatal and postnatal period coupled with the failure to make decisions within the household can translate into a greater risk of mortality for male children, owing to the differences in their genetic and biological makeup, which results in them being more susceptible to diseases than female children. 


\subsection{Child Marriage and the Status of Women in the Household}

The objective in this section is to examine the relationship between the status of women in the household and Child Marriage. There is great variation in measuring the status of women. In the literature, the status of women has been determined by their economic power, freedom from the control of family members (autonomy), and access to and control over material and social resources (Mason 1986, Dyson and Moore 1983, Dixon-Mueller 2013). A woman's economic power is reliant on present-day economic resources and past resources that were brought in at the time of the marriage (Frankenberg and Thomas 2001, Doss 1997, Lundberg et al. 1997). As the data on economic resources (assets, unearned income, welfare and transfer payments) is unavailable, I focus instead upon assessing the status of women using measures of autonomy. Following Basu (1992) and Dyson and Moore (1983), I characterize autonomy as the capacity of the woman to control household resources, the power to exert influence over decisions about themselves and family members and the ability to have freedom of movement.

An advantage of the Bangladesh Demographic and Health Survey is the provision of information from the Women's Questionnaire on women's participation in household decision-making. I specifically choose questions from the survey that are related to the ability of the woman to influence the health outcomes of her children. I use two sets of criteria to characterize the women's status in the household: the ability of the woman to access health care for herself and her degree of decision-making autonomy in the household. I assess the ability of the woman to access health care from the following outcomes: whether the woman goes outside the place of residence alone, whether the woman goes to the health center or hospital alone, whether the woman has a problem with 
getting permission to go to the health facility and whether the woman has a problem with getting money for treatment. To measure women's decision-making autonomy, I use outcomes based on whether the woman has a final say in decisions regarding her own health care, child health care, large household purchases, household purchases for daily needs, visits to family, friends or relatives and the type of food that should be cooked daily. Indicator variables are constructed for each question, where a value of 1 is assigned if the woman makes all final say decisions independently, can freely move and experiences problems receiving money for treatment and permission to visit the health facility. Otherwise, the variable is given a value of 0 .

To investigate the effect of Child Marriage on the status of women in the household, I use the following equation:

$Y_{i, c}=\delta_{1}+\delta_{2}$ ChildMarriage $_{i, c}+\delta_{3} Z_{i, c}+\omega_{c}+\varepsilon_{i, c}$

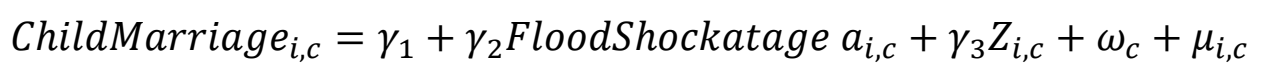

where the dependent variable $Y_{i, c}$ represents each of the 11 indicator variables used to measure the status of woman $i$ in cluster $c$. The coefficient of interest is $\delta_{2}$, the impact of instrumented Child Marriage on the autonomy outcome. The vector $Z_{i, c}$ contains controls that cover the educational level of the woman and her husband, the age of the woman, the type of location (urban or rural) and the level of household wealth. Cluster fixed effects $\omega_{c}$ are employed to capture unobserved characteristics in clusters. The instrument, FloodShockatage $a_{i, c}$ is a binary indicator for whether woman $i$ in cluster $c$ experienced a flood shock at age $a$. For example, if woman $i$ in cluster c experienced a flood shock at age 13, the binary indicator is coded as 1 ; otherwise it is 0 . Given that the focus is on Child Marriages, ages (a) from 12 to 17 are used in the analysis. I estimate two different 
specifications: an IV regression characterizing the ability of the woman to access healthcare for self; and an IV regression characterizing the woman's degree of decisionmaking autonomy in the household.

Table 2.10 reports the results characterizing the effect of Child Marriage on the ability of the woman to access health care for herself. In columns 1 to 4 , flood shocks experienced across age ranges 12 to 13,14 to 15 and 16 to 17 are used as instrumental variables. Standard errors are presented in parentheses, and size of the regression along with the Sargan p-value and Cragg Donald F-statistic are in the last three rows of the table. The positive and statistically significant coefficient on the Child Marriage estimate in column 3 implies that child brides have a higher probability of experiencing a problem in gaining permission to go to the health facility. Correspondingly, the negative coefficient on the Child Marriage estimate in column 2 indicates that being married as a child decreases the probability of the woman going to the hospital alone by 67.9 percentage points. The coefficient is similar in magnitude but has less statistical power in comparison to column 3; this may be due to the smaller sample size which resulted from missing data. Women from Child Marriages in urban areas have a significant negative coefficient (column 3) indicating that they have fewer problems in gaining permission to go to the health facility compared to those in rural areas. This is expected as the hospital or health facility may be closer for women in urban areas and thereby more accessible and less costly to reach. The coefficient on age is significantly correlated across all specifications, denoting that as the age of women in Child Marriages increases, their access to the outside increases. In addition, they have fewer problems in getting permission to go to the health facility and getting money for treatment. 
These estimates suggest that Child Marriage substantially lowers the ability of the woman to access healthcare. However, there is little indication that going outside alone or having problems getting permission for money for treatment are affected by Child Marriage. The coefficients on Child Marriage in columns 1 and 4 are smaller in magnitude than the corresponding coefficients in the regressions in columns 2 and 3, and none are statistically different from zero.

The IV results for the effect of Child Marriage on decision-making autonomy in the household are presented in Table 2.11. The average of flood shocks across ages 12 to 17 is used as an instrument for Child Marriage. The estimation results show that Child Marriage has a mixed effect on decision-making, depending on the type of decision. In particular, I find that Child Marriage significantly decreases the probability that a woman makes decisions on her health care, her children's health care, daily purchases in the household and the type of food to be cooked daily. On the other hand, Child Marriage has no effect on decisions on large household purchases or visits to family and friends; the coefficient on the Child Marriage estimate is small in magnitude and is insignificant (columns 3 and 5). It is worth noting that the coefficients on age and urban location are positive and significantly correlated with the probability of decision-making. This corresponds with the previous finding in Table 2.10. As child brides grow older, they gain more knowledge of their new household and adapt according to the demands. A child bride can accede to a higher status over time from fulfilling her child-bearing duties or from the death of in-laws (Guilbert 2013). Women living in urban areas face fewer sociocultural restrictions and have greater access to non-traditional employment opportunities than those in rural areas (Pozarny 2016), which may serve to positively influence their decision-making power. 
The results presented are indicative of the low power of the child bride within the household. Child Marriage significantly impacts the ability of the woman to access health care and to make healthcare decisions for herself and her children.

\subsection{Conclusion}

Ultimately, any progress towards child survival will require improvements in the health care system, such as expansive immunization coverage and greater access to obstetric care. However, the results in this paper suggest that Child Marriage also plays an important role in determining the survival outcomes for children.

In this paper, I explore the effect of Child Marriage on the mortality outcomes of children produced from these unions. Establishing a link is challenging since Child Marriage and child mortality may be associated with omitted variables. To address endogeneity concerns, I develop and implement an instrumental variable strategy. I exploit the exogenous variation in rainfall shocks over the years the mother was at ages 12 to 17 to use as an instrument for Child Marriage.

This paper provides compelling evidence that the practice of Child Marriage contributes significantly to increases in child mortality and infant mortality in Bangladesh. I find that the effect of Child Marriage is larger for child mortality than for infant mortality. By examining the effect by gender, the results suggest that male children from Child Marriage unions face a higher likelihood of dying before the age of five than female children. In addition, the results indicate that Child Marriage does not seem to influence the outcomes of female infant mortality, implying that the predominant effect of Child Marriage is concentrated on increases in male infant mortality. 
There are several channels through which Child Marriage can affect the mortality outcomes of children. I present results that suggest that the lower status of women in the groom's household may be a probable channel. The results indicate that Child Marriage unions reduce women's decision-making power and access to health care. I find that women who are married before the age of 18 are 51 percentage points more likely to have problems gaining permission to visit the facility, 36.6 percentage points less likely to make their own health care decisions and 35.4 percentage points less likely to be allowed to make decisions on their children's health care.

This paper differs from the existing literature in that it focuses on the impact of an exogenous temporary shock on Child Marriage, allowing for the identification of causal impact. The results have important implications for policy. The substantial levels of child and infant mortality among mothers who were married before the age of 18 clearly indicate that designing effective policies aimed at combating child mortality requires additional consideration for children from Child Marriage unions. Additionally, the findings of the effect on women's status highlight the need for programmatic efforts to empower women who were married early and suggest that such efforts may play an important role in influencing the outcomes of children from Child Marriage unions. 
Figure 2.1: Child Mortality by Gender

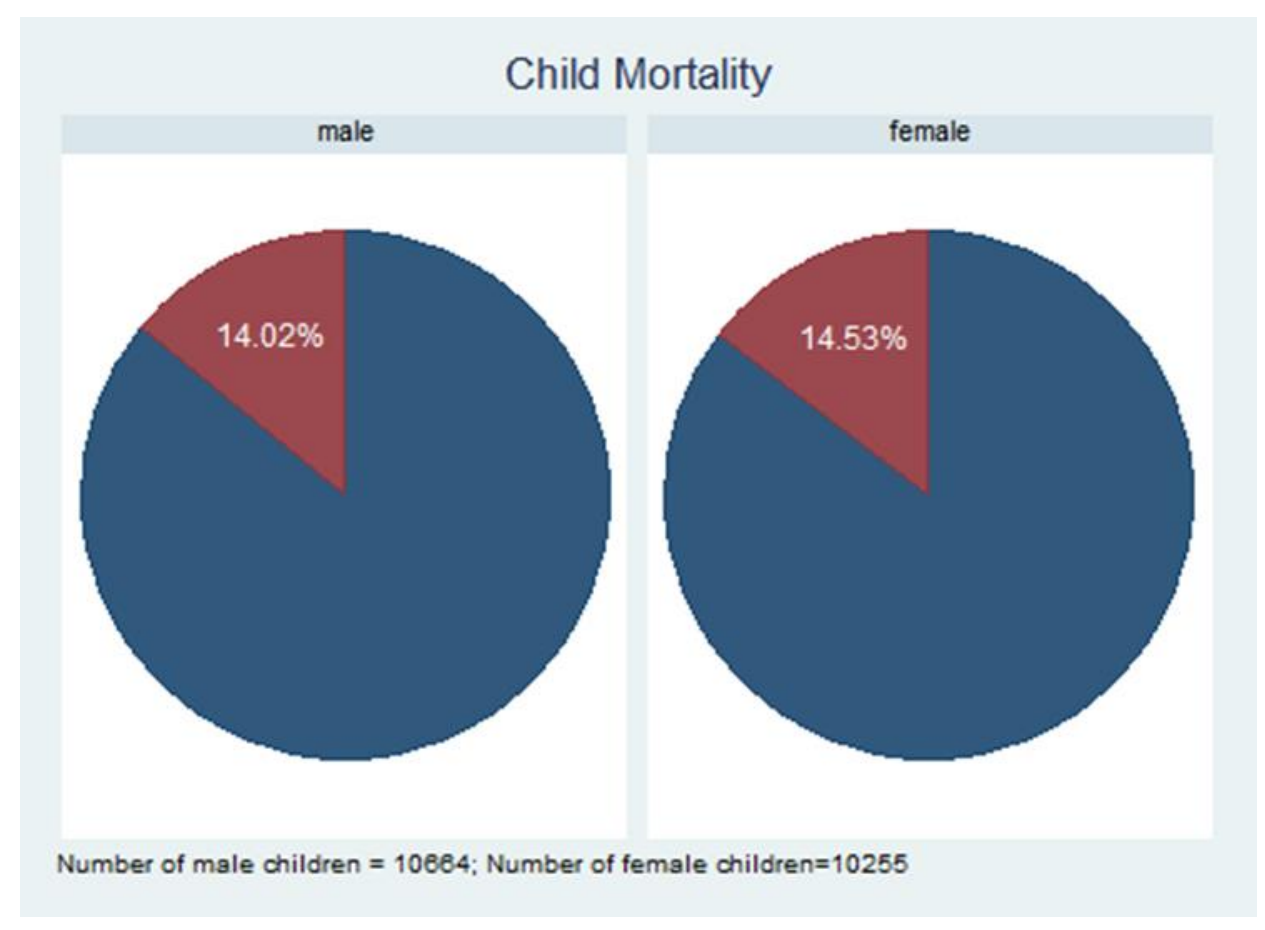


Figure 2.2: Infant Mortality by Gender

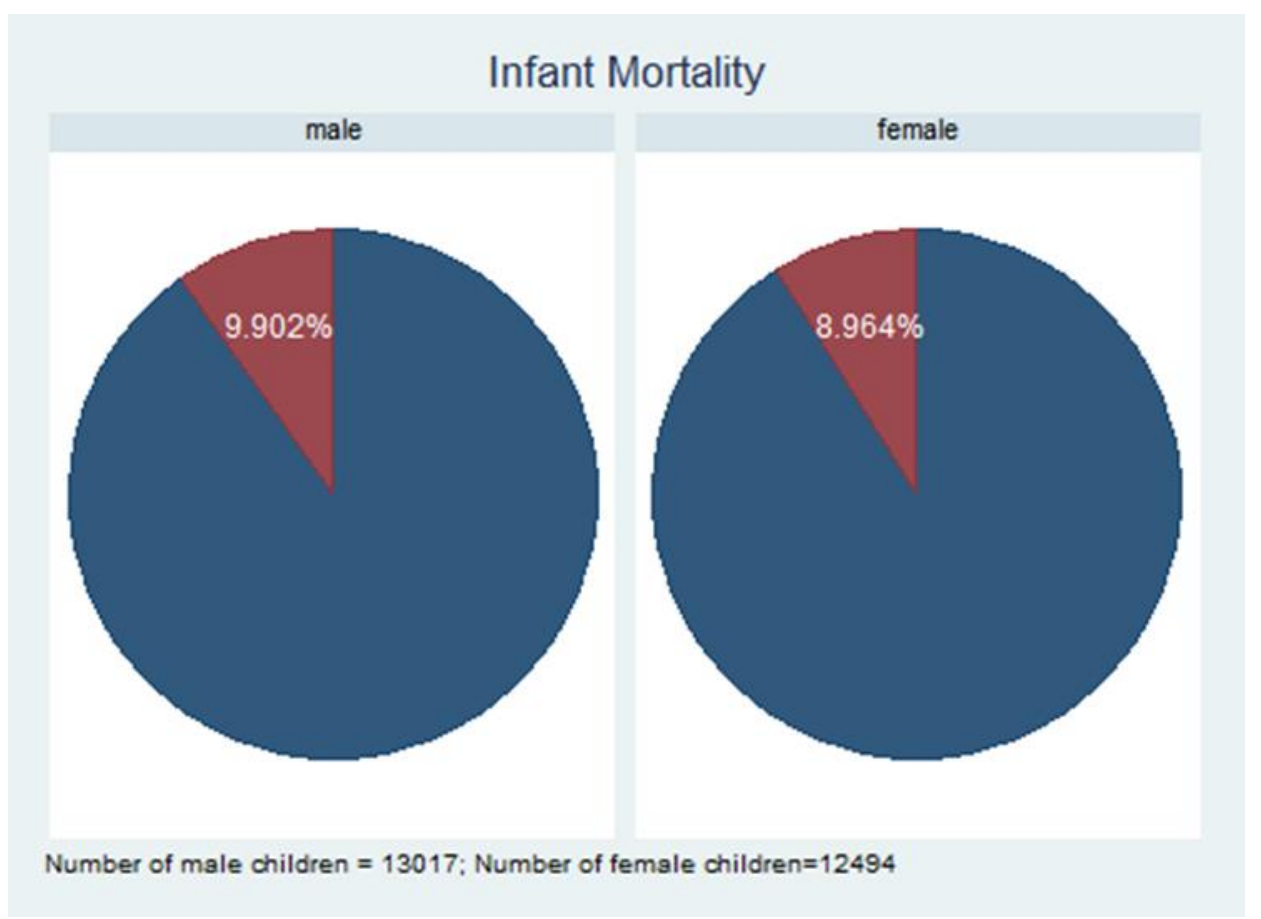


Figure 2.3: Child Mortality by Birth Order

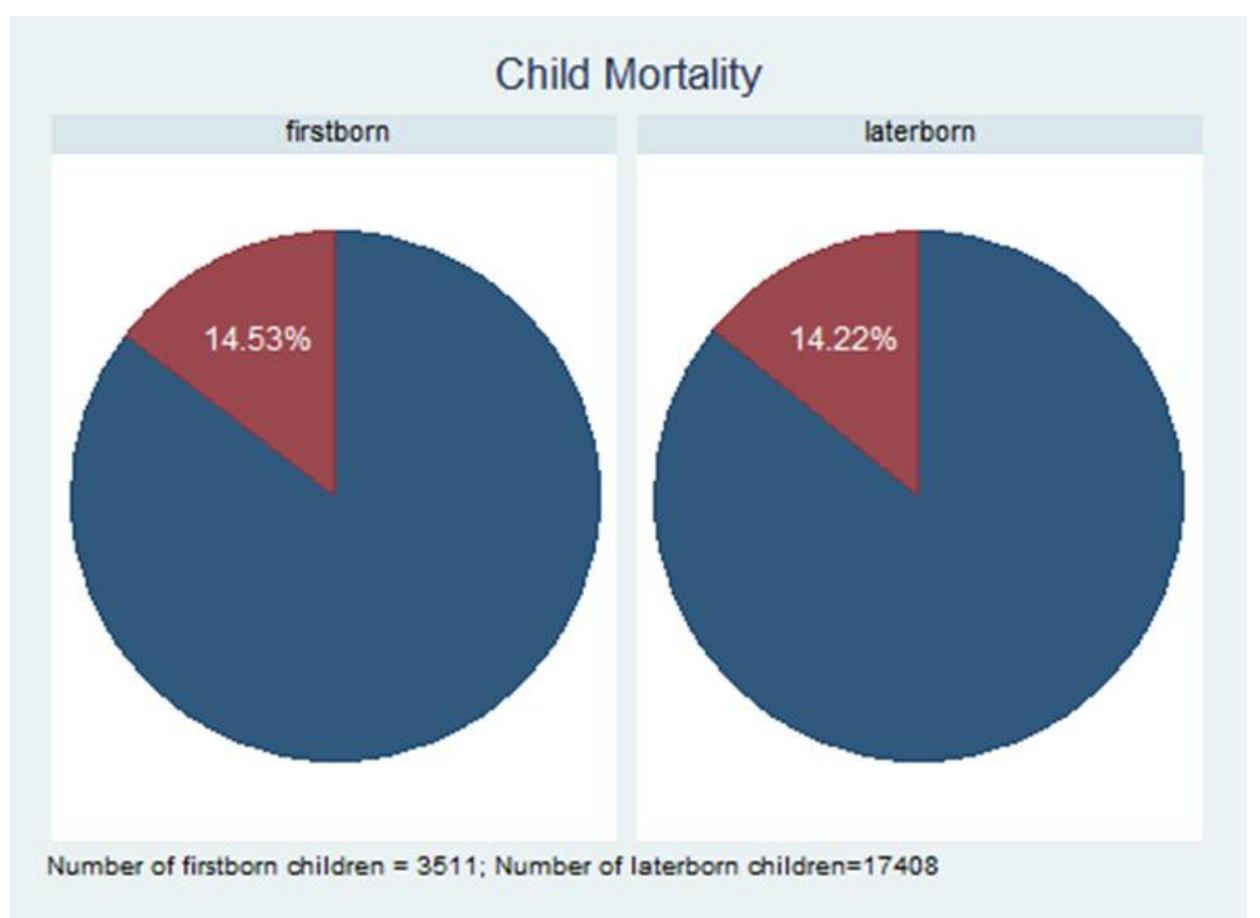


Figure 2.4: Infant Mortality by Birth Order

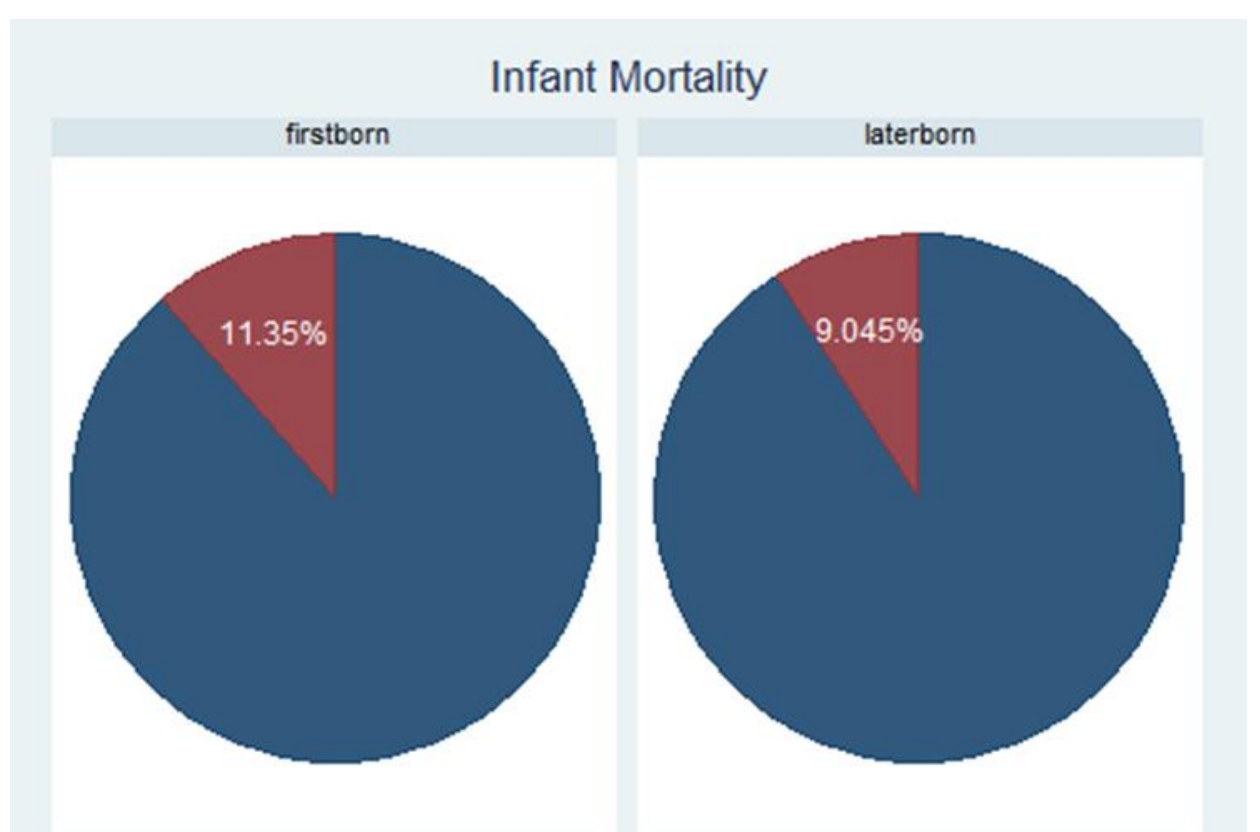

Number of firstborn children $=4395$; Number of laterborn children=21116 
Table 2.1: Summary Statistics for Child Mortality Sample

\begin{tabular}{lcccc}
\hline & Mean & Std. Dev & Min & Max \\
\hline & & & & \\
By Mother & & & & \\
Age at marriage & 14.443 & 2.64 & 6 & 39 \\
Child Marriage & 0.882 & 0.32 & 0 & 1 \\
Married before 15 & 0.583 & 0.49 & 0 & 1 \\
Age at Birth & 24.171 & 4.99 & 18 & 45 \\
& & & & \\
By Education & & & & \\
Mother- Primary Edu & 0.450 & 0.50 & 0 & 1 \\
Father- Primary Edu & 0.575 & 0.49 & 0 & 1 \\
& & & & \\
By Household & & & & \\
Urban & 0.272 & 0.44 & 0 & 1 \\
Nuclear Family & 0.798 & 0.40 & 0 & 1 \\
Highest Quintile & 0.235 & 0.42 & 0 & 1 \\
Fourth Quintile & 0.202 & 0.40 & 0 & 1 \\
Second Quintile & 0.189 & 0.39 & 0 & 1 \\
Lowest Quintile & 0.182 & 0.39 & 0 & 1 \\
& & & & \\
By Children & & & & \\
Children in HH & \\
Birth Interval & 3.076 & 1.96 & 0 & 11 \\
Firstborn & 0.109 & 0.31 & 0 & 1 \\
Female & 0.168 & 0.37 & 0 & 1 \\
Male & 0.490 & 0.50 & 0 & 1 \\
Child Mortality & 0.510 & 0.50 & 0 & 1 \\
\hline Observations & 0.143 & 0.35 & 0 & 1 \\
\hline
\end{tabular}

${ }^{24}$ Surviving children in the household at the time of the child's birth 
Table 2.2: Summary Statistics for Infant Mortality Sample

\begin{tabular}{lcccc}
\hline & Mean & Std. Dev & Min & Max \\
\hline & & & & \\
By Mother & & & & \\
Age at marriage & 14.618 & 2.74 & 6 & 39 \\
Child Marriage & 0.866 & 0.34 & 0 & 1 \\
Married before 15 & 0.560 & 0.50 & 0 & 1 \\
Age at Birth & 24.376 & 5.12 & 18 & 47 \\
& & & & \\
By Education & & & & \\
Mother- Primary Edu & 0.464 & 0.50 & 0 & 1 \\
Father- Primary Edu & 0.574 & 0.49 & 0 & 1 \\
& & & & \\
By Household & & & & \\
Urban & 0.269 & 0.44 & 0 & 1 \\
Nuclear Family & 0.785 & 0.41 & 0 & 1 \\
Highest Quintile & 0.230 & 0.42 & 0 & 1 \\
Fourth Quintile & 0.197 & 0.40 & 0 & 1 \\
Second Quintile & 0.191 & 0.39 & 0 & 1 \\
Lowest Quintile & 0.193 & 0.39 & 0 & 1 \\
& & & & \\
By Children & & & & \\
Children in HH & 3.105 & 1.98 & 0 & 11 \\
Birth Interval & 0.100 & 0.30 & 0 & 1 \\
Firstborn & 0.172 & 0.38 & 0 & 1 \\
Female & 0.490 & 0.50 & 0 & 1 \\
Male & 0.510 & 0.50 & 0 & 1 \\
Infant Mortality & 0.094 & 0.29 & 0 & 1 \\
\hline Observations & 25511 & & & \\
\hline
\end{tabular}


Table 2.3: OLS Results - Child Mortality and Infant Mortality

\begin{tabular}{|c|c|c|c|c|}
\hline & $\begin{array}{c}\text { (1) } \\
\text { OLS } \\
\text { deadbefore5 }\end{array}$ & $\begin{array}{c}\text { (2) } \\
\text { OLS } \\
\text { deadbefore5 }\end{array}$ & $\begin{array}{c}\text { (3) } \\
\text { OLS } \\
\text { deadbefore1 } \\
\end{array}$ & $\begin{array}{c}\text { (4) } \\
\text { OLS } \\
\text { deadbefore1 } \\
\end{array}$ \\
\hline Child Marriage & $\begin{array}{l}0.0314^{* * *} \\
(0.00768)\end{array}$ & $\begin{array}{l}0.0367^{* * *} \\
(0.00773)\end{array}$ & $\begin{array}{l}0.0198^{* * *} \\
(0.00557)\end{array}$ & $\begin{array}{l}0.0236^{* * *} \\
(0.00560)\end{array}$ \\
\hline Age at Birth & $\begin{array}{l}0.0000721 \\
(0.000531)\end{array}$ & $\begin{array}{l}-0.000226 \\
(0.000532)\end{array}$ & $\begin{array}{c}0.000110 \\
(0.000396)\end{array}$ & $\begin{array}{l}-0.000147 \\
(0.000397)\end{array}$ \\
\hline Highest Quintile & $\begin{array}{l}-0.0275^{* * *} \\
(0.00823)\end{array}$ & $\begin{array}{l}-0.0286^{* * *} \\
(0.00830)\end{array}$ & $\begin{array}{l}-0.0153^{* *} \\
(0.00629)\end{array}$ & $\begin{array}{l}-0.0180^{* * *} \\
(0.00634)\end{array}$ \\
\hline Fourth Quintile & $\begin{array}{c}-0.0103 \\
(0.00768)\end{array}$ & $\begin{array}{c}-0.0118 \\
(0.00768)\end{array}$ & $\begin{array}{l}-0.00899 \\
(0.00589)\end{array}$ & $\begin{array}{l}-0.00980^{*} \\
(0.00589)\end{array}$ \\
\hline Lowest Quintile & $\begin{array}{l}0.0330^{* * *} \\
(0.00807)\end{array}$ & $\begin{array}{l}0.0313^{* * *} \\
(0.00807)\end{array}$ & $\begin{array}{c}0.0108^{*} \\
(0.00608)\end{array}$ & $\begin{array}{c}0.00857 \\
(0.00608)\end{array}$ \\
\hline Second Quintile & $\begin{array}{l}0.0248^{* * *} \\
(0.00779)\end{array}$ & $\begin{array}{l}0.0260^{* * *} \\
(0.00779)\end{array}$ & $\begin{array}{c}0.0123^{* *} \\
(0.00593)\end{array}$ & $\begin{array}{c}0.0125^{* *} \\
(0.00592)\end{array}$ \\
\hline Father-Primary Edu & $\begin{array}{l}-0.00880 \\
(0.00563)\end{array}$ & $\begin{array}{l}-0.00851 \\
(0.00565)\end{array}$ & $\begin{array}{l}-0.00559 \\
(0.00429)\end{array}$ & $\begin{array}{l}-0.00508 \\
(0.00430)\end{array}$ \\
\hline Mother-Primary Edu & $\begin{array}{l}-0.0321^{* * *} \\
(0.00569)\end{array}$ & $\begin{array}{l}-0.0310^{* * *} \\
(0.00577)\end{array}$ & $\begin{array}{c}-0.0218^{* * *} \\
(0.00433)\end{array}$ & $\begin{array}{l}-0.0195^{* * *} \\
(0.00438)\end{array}$ \\
\hline Urban & $\begin{array}{l}-0.00770 \\
(0.00619)\end{array}$ & $\begin{array}{l}-0.00754 \\
(0.00621)\end{array}$ & $\begin{array}{l}-0.00403 \\
(0.00471)\end{array}$ & $\begin{array}{l}-0.00231 \\
(0.00473)\end{array}$ \\
\hline Nuclear Family & $\begin{array}{l}-0.00563 \\
(0.00594)\end{array}$ & $\begin{array}{l}-0.00265 \\
(0.00596)\end{array}$ & $\begin{array}{l}-0.000151 \\
(0.00444)\end{array}$ & $\begin{array}{c}0.00103 \\
(0.00446)\end{array}$ \\
\hline Children in $\mathrm{HH}$ & $\begin{array}{l}-0.0397^{* * *} \\
(0.00233)\end{array}$ & $\begin{array}{l}-0.0391^{* * *} \\
(0.00234)\end{array}$ & $\begin{array}{c}-0.0312^{* * *} \\
(0.00175)\end{array}$ & $\begin{array}{l}-0.0304^{* * *} \\
(0.00176)\end{array}$ \\
\hline Birth Interval & $\begin{array}{l}0.0895^{* * *} \\
(0.00765)\end{array}$ & $\begin{array}{c}0.0880^{* * *} \\
(0.00765)\end{array}$ & $\begin{array}{l}0.0752^{* * *} \\
(0.00605)\end{array}$ & $\begin{array}{c}0.0737^{* * *} \\
(0.00604)\end{array}$ \\
\hline FE & no & yes & no & Yes \\
\hline F-Stat & 61.86 & 61.25 & 55.01 & 53.07 \\
\hline R2 & 0.0343 & 0.0340 & 0.0252 & 0.0244 \\
\hline Observations & 20919 & 20919 & 25511 & 25511 \\
\hline
\end{tabular}

Standard errors in parentheses

OLS

${ }^{*} p<.1,{ }^{* *} p<.05,{ }^{* * *} p<.01$ 
Table 2.4: First-stage IV Results - Child Mortality

\begin{tabular}{|c|c|c|c|c|}
\hline & $\begin{array}{c}\text { (1) } \\
\text { Child Marriage }\end{array}$ & $\begin{array}{c}(2) \\
\text { Child Marriage }\end{array}$ & $\begin{array}{c}\text { (3) } \\
\text { Child Marriage }\end{array}$ & $\begin{array}{c}\text { (4) } \\
\text { Child Marriage }\end{array}$ \\
\hline FS -12 to 13 & $\begin{array}{l}-0.0219^{* * *} \\
(0.00846)\end{array}$ & $\begin{array}{l}-0.0311^{* * *} \\
(0.00849)\end{array}$ & & \\
\hline FS -14 to 15 & $\begin{array}{l}-0.00657 \\
(0.00836)\end{array}$ & $\begin{array}{l}-0.0213^{* *} \\
(0.00846)\end{array}$ & & \\
\hline FS -16 to 17 & $\begin{array}{c}-0.0214^{* * *} \\
(0.00808)\end{array}$ & $\begin{array}{l}-0.0396^{* * *} \\
(0.00815)\end{array}$ & & \\
\hline Age at Birth & $\begin{array}{c}-0.0114^{* * *} \\
(0.000476)\end{array}$ & $\begin{array}{c}-0.0109^{* * *} \\
(0.000474)\end{array}$ & $\begin{array}{l}-0.0114^{* * *} \\
(0.000476)\end{array}$ & $\begin{array}{c}-0.0109^{* * *} \\
(0.000474)\end{array}$ \\
\hline Highest Quintile & $\begin{array}{c}-0.0734^{* * *} \\
(0.00739)\end{array}$ & $\begin{array}{l}-0.0641^{* * *} \\
(0.00740)\end{array}$ & $\begin{array}{l}-0.0734^{* * * *} \\
(0.00739)\end{array}$ & $\begin{array}{l}-0.0643^{* * *} \\
(0.00740)\end{array}$ \\
\hline Fourth Quintile & $\begin{array}{l}-0.0143^{* *} \\
(0.00691)\end{array}$ & $\begin{array}{l}-0.00823 \\
(0.00687)\end{array}$ & $\begin{array}{l}-0.0145^{* *} \\
(0.00691)\end{array}$ & $\begin{array}{l}-0.00847 \\
(0.00686)\end{array}$ \\
\hline Lowest Quintile & $\begin{array}{l}-0.0247^{* * *} \\
(0.00727)\end{array}$ & $\begin{array}{l}-0.0178^{* *} \\
(0.00722)\end{array}$ & $\begin{array}{l}-0.0247^{* * *} \\
(0.00726)\end{array}$ & $\begin{array}{l}-0.0180^{* *} \\
(0.00722)\end{array}$ \\
\hline Second Quintile & $\begin{array}{l}-0.0144^{* *} \\
(0.00702)\end{array}$ & $\begin{array}{l}-0.0144^{* *} \\
(0.00696)\end{array}$ & $\begin{array}{l}-0.0144^{* *} \\
(0.00702)\end{array}$ & $\begin{array}{l}-0.0145^{* *} \\
(0.00696)\end{array}$ \\
\hline Father-Primary Edu & $\begin{array}{c}-0.0335^{* * *} \\
(0.00507)\end{array}$ & $\begin{array}{l}-0.0335^{* * *} \\
(0.00504)\end{array}$ & $\begin{array}{l}-0.0335^{* * *} \\
(0.00507)\end{array}$ & $\begin{array}{l}-0.0335^{* * *} \\
(0.00504)\end{array}$ \\
\hline Mother-Primary Edu & $\begin{array}{l}-0.0670^{* * *} \\
(0.00510)\end{array}$ & $\begin{array}{l}-0.0752^{* * *} \\
(0.00513)\end{array}$ & $\begin{array}{l}-0.0670^{* * *} \\
(0.00510)\end{array}$ & $\begin{array}{c}-0.0751^{* * *} \\
(0.00513)\end{array}$ \\
\hline Urban & $\begin{array}{l}-0.0386^{* * *} \\
(0.00556)\end{array}$ & $\begin{array}{l}-0.0376^{* * *} \\
(0.00554)\end{array}$ & $\begin{array}{l}-0.0386^{* * *} \\
(0.00556)\end{array}$ & $\begin{array}{l}-0.0375^{* * *} \\
(0.00554)\end{array}$ \\
\hline Nuclear Family & $\begin{array}{c}0.00174 \\
(0.00535)\end{array}$ & $\begin{array}{l}-0.00606 \\
(0.00533)\end{array}$ & $\begin{array}{c}0.00158 \\
(0.00535)\end{array}$ & $\begin{array}{c}-0.00618 \\
(0.00533)\end{array}$ \\
\hline Children in $\mathrm{HH}$ & $\begin{array}{l}0.0537^{* * *} \\
(0.00207)\end{array}$ & $\begin{array}{l}0.0530^{* * *} \\
(0.00206)\end{array}$ & $\begin{array}{l}0.0538^{* * *} \\
(0.00207)\end{array}$ & $\begin{array}{l}0.0530^{* * *} \\
(0.00206)\end{array}$ \\
\hline Birth Interval & $\begin{array}{c}0.00120 \\
(0.00689)\end{array}$ & $\begin{array}{c}0.00483 \\
(0.00684)\end{array}$ & $\begin{array}{c}0.00115 \\
(0.00689)\end{array}$ & $\begin{array}{c}0.00481 \\
(0.00684)\end{array}$ \\
\hline Flood shocks - 12 to 17 & & & $\begin{array}{c}-0.0514^{* * *} \\
(0.0148)\end{array}$ & $\begin{array}{c}-0.0940^{* * *} \\
(0.0153)\end{array}$ \\
\hline
\end{tabular}




\begin{tabular}{lcccc}
\hline FE & no & yes & no & yes \\
F-Stat & 127.2 & 127.9 & 148.2 & 149.0 \\
R2 & 0.0785 & 0.0789 & 0.0784 & 0.0788 \\
Observations & 20919 & 20919 & 20919 & 20919 \\
\hline
\end{tabular}

Standard errors in parentheses

OLS

${ }^{*} p<.1,{ }^{* *} p<.05,{ }^{* * *} p<.01$ 
Table 2.5: First-stage IV Results - Infant Mortality

\begin{tabular}{|c|c|c|c|c|}
\hline & $\begin{array}{c}\text { (1) } \\
\text { Child Marriage }\end{array}$ & $\begin{array}{c}\text { (2) } \\
\text { Child Marriage }\end{array}$ & $\begin{array}{c}\text { (3) } \\
\text { Child Marriage }\end{array}$ & $\begin{array}{c}\text { (4) } \\
\text { Child Marriage }\end{array}$ \\
\hline FS -12 to 13 & $\begin{array}{l}-0.0364^{* * *} \\
(0.00789)\end{array}$ & $\begin{array}{l}-0.0469^{* * *} \\
(0.00792)\end{array}$ & & \\
\hline FS -14 to 15 & $\begin{array}{c}-0.0122 \\
(0.00790)\end{array}$ & $\begin{array}{l}-0.0255^{* * *} \\
(0.00797)\end{array}$ & & \\
\hline FS -16 to 17 & $\begin{array}{l}-0.0166^{* *} \\
(0.00774)\end{array}$ & $\begin{array}{l}-0.0333^{* * *} \\
(0.00778)\end{array}$ & & \\
\hline Age at Birth & $\begin{array}{c}-0.0125^{* * *} \\
(0.000440)\end{array}$ & $\begin{array}{l}-0.0119^{* * *} \\
(0.000438)\end{array}$ & $\begin{array}{l}-0.0125^{* * *} \\
(0.000440)\end{array}$ & $\begin{array}{l}-0.0119^{* * *} \\
(0.000438)\end{array}$ \\
\hline Highest Quintile & $\begin{array}{l}-0.0766^{* * *} \\
(0.00706)\end{array}$ & $\begin{array}{l}-0.0675^{* * *} \\
(0.00707)\end{array}$ & $\begin{array}{l}-0.0763^{* * *} \\
(0.00706)\end{array}$ & $\begin{array}{l}-0.0673^{* * *} \\
(0.00707)\end{array}$ \\
\hline Fourth Quintile & $\begin{array}{l}-0.0113^{*} \\
(0.00662)\end{array}$ & $\begin{array}{l}-0.00508 \\
(0.00658)\end{array}$ & $\begin{array}{l}-0.0113^{*} \\
(0.00662)\end{array}$ & $\begin{array}{l}-0.00515 \\
(0.00658)\end{array}$ \\
\hline Lowest Quintile & $\begin{array}{l}-0.0206^{* * *} \\
(0.00684)\end{array}$ & $\begin{array}{l}-0.0139^{* *} \\
(0.00680)\end{array}$ & $\begin{array}{l}-0.0204^{* * *} \\
(0.00684)\end{array}$ & $\begin{array}{l}-0.0137^{* *} \\
(0.00680)\end{array}$ \\
\hline Second Quintile & $\begin{array}{l}-0.00874 \\
(0.00667)\end{array}$ & $\begin{array}{l}-0.00880 \\
(0.00662)\end{array}$ & $\begin{array}{l}-0.00870 \\
(0.00667)\end{array}$ & $\begin{array}{l}-0.00878 \\
(0.00662)\end{array}$ \\
\hline Father-Primary Edu & $\begin{array}{l}-0.0371^{* * *} \\
(0.00482)\end{array}$ & $\begin{array}{l}-0.0371^{* * *} \\
(0.00480)\end{array}$ & $\begin{array}{l}-0.0370^{* * *} \\
(0.00482)\end{array}$ & $\begin{array}{l}-0.0370^{* * *} \\
(0.00480)\end{array}$ \\
\hline Mother-Primary Edu & $\begin{array}{l}-0.0785^{* * *} \\
(0.00484)\end{array}$ & $\begin{array}{l}-0.0869^{* * *} \\
(0.00487)\end{array}$ & $\begin{array}{l}-0.0787^{* * *} \\
(0.00484)\end{array}$ & $\begin{array}{l}-0.0871^{* * *} \\
(0.00487)\end{array}$ \\
\hline urban & $\begin{array}{l}-0.0381^{* * *} \\
(0.00529)\end{array}$ & $\begin{array}{l}-0.0375^{* * *} \\
(0.00528)\end{array}$ & $\begin{array}{l}-0.0382^{* * *} \\
(0.00529)\end{array}$ & $\begin{array}{l}-0.0376^{* * *} \\
(0.00528)\end{array}$ \\
\hline Nuclear Family & $\begin{array}{l}0.0283^{* * *} \\
(0.00500)\end{array}$ & $\begin{array}{l}0.0207^{* * *} \\
(0.00498)\end{array}$ & $\begin{array}{l}0.0282^{* * *} \\
(0.00500)\end{array}$ & $\begin{array}{l}0.0206^{* * *} \\
(0.00498)\end{array}$ \\
\hline Children in $\mathrm{HH}$ & $\begin{array}{l}0.0588^{* * *} \\
(0.00193)\end{array}$ & $\begin{array}{l}0.0586^{* * *} \\
(0.00193)\end{array}$ & $\begin{array}{c}0.0589^{* * *} \\
(0.00193)\end{array}$ & $\begin{array}{c}0.0587^{* * *} \\
(0.00193)\end{array}$ \\
\hline Birth Interval & $\begin{array}{c}0.00316 \\
(0.00680)\end{array}$ & $\begin{array}{c}0.00702 \\
(0.00675)\end{array}$ & $\begin{array}{c}0.00318 \\
(0.00680)\end{array}$ & $\begin{array}{c}0.00703 \\
(0.00675)\end{array}$ \\
\hline Flood shocks - 12 to 17 & & & $\begin{array}{c}-0.0661^{* * *} \\
(0.0136)\end{array}$ & $\begin{array}{l}-0.107^{* * *} \\
(0.0141)\end{array}$ \\
\hline
\end{tabular}




\begin{tabular}{lcccc}
\hline FE & no & yes & no & yes \\
F-Stat & 183.7 & 185.0 & 213.8 & 215.5 \\
R2 & 0.0916 & 0.0922 & 0.0914 & 0.0921 \\
Observations & 25511 & 25511 & 25511 & 25511 \\
\hline
\end{tabular}

Standard errors in parentheses

OLS

${ }^{*} p<.1,{ }^{* *} p<.05,{ }^{* * *} p<.01$ 
Table 2.6: IV Results - Child Mortality

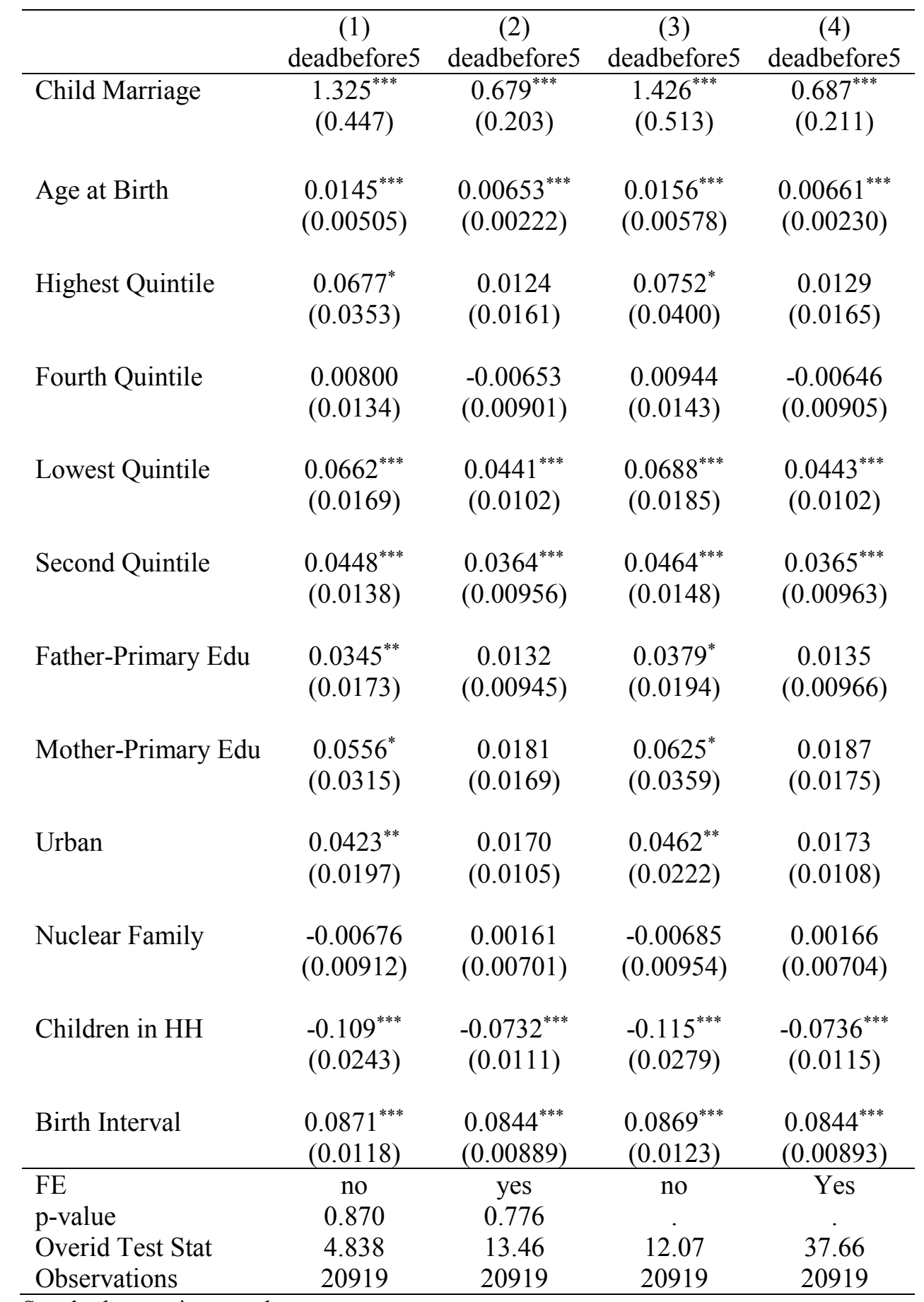

Standard errors in parentheses

Columns 1 and 2: flood shocks across ages 12 to 13,14 to 15 and 16 to 17 are used as IVs. Column 3 and 4 use the average of flood shocks across the ages 12 to 17 as an IV.

${ }^{*} p<.1,{ }^{* *} p<.05,{ }^{* * *} p<.01$ 
Table 2.7: IV Results - Infant Mortality

\begin{tabular}{|c|c|c|c|c|}
\hline & $\begin{array}{c}(1) \\
\text { deadbefore1 }\end{array}$ & $\begin{array}{c}(2) \\
\text { deadbefore1 }\end{array}$ & $\begin{array}{c}(3) \\
\text { deadbefore1 }\end{array}$ & $\begin{array}{c}\text { (4) } \\
\text { deadbefore1 }\end{array}$ \\
\hline Child Marriage & $\begin{array}{l}0.444^{* *} \\
(0.183)\end{array}$ & $\begin{array}{l}0.248^{* *} \\
(0.118)\end{array}$ & $\begin{array}{c}0.361^{*} \\
(0.197)\end{array}$ & $\begin{array}{c}0.205^{*} \\
(0.120)\end{array}$ \\
\hline Age at Birth & $\begin{array}{l}0.00534^{* *} \\
(0.00230)\end{array}$ & $\begin{array}{c}0.00248^{*} \\
(0.00143)\end{array}$ & $\begin{array}{c}0.00433^{*} \\
(0.00246)\end{array}$ & $\begin{array}{c}0.00198 \\
(0.00146)\end{array}$ \\
\hline Highest Quintile & $\begin{array}{c}0.0172 \\
(0.0157)\end{array}$ & $\begin{array}{l}-0.00290 \\
(0.0102)\end{array}$ & $\begin{array}{c}0.0109 \\
(0.0165)\end{array}$ & $\begin{array}{l}-0.00575 \\
(0.0103)\end{array}$ \\
\hline Fourth Quintile & $\begin{array}{l}-0.00437 \\
(0.00682)\end{array}$ & $\begin{array}{l}-0.00871 \\
(0.00610)\end{array}$ & $\begin{array}{l}-0.00527 \\
(0.00666)\end{array}$ & $\begin{array}{c}-0.00892 \\
(0.00604)\end{array}$ \\
\hline Lowest Quintile & $\begin{array}{c}0.0199^{* *} \\
(0.00781)\end{array}$ & $\begin{array}{c}0.0121^{*} \\
(0.00654)\end{array}$ & $\begin{array}{c}0.0181^{* *} \\
(0.00777)\end{array}$ & $\begin{array}{c}0.0115^{*} \\
(0.00650)\end{array}$ \\
\hline Second Quintile & $\begin{array}{c}0.0165^{* *} \\
(0.00681)\end{array}$ & $\begin{array}{c}0.0148^{* *} \\
(0.00623)\end{array}$ & $\begin{array}{c}0.0157^{* *} \\
(0.00664)\end{array}$ & $\begin{array}{c}0.0144^{* *} \\
(0.00618)\end{array}$ \\
\hline Father-Primary Edu & $\begin{array}{c}0.0101 \\
(0.00829)\end{array}$ & $\begin{array}{c}0.00333 \\
(0.00625)\end{array}$ & $\begin{array}{c}0.00706 \\
(0.00861)\end{array}$ & $\begin{array}{c}0.00174 \\
(0.00629)\end{array}$ \\
\hline Mother-Primary Edu & $\begin{array}{c}0.0118 \\
(0.0153)\end{array}$ & $\begin{array}{c}0.000239 \\
(0.0113)\end{array}$ & $\begin{array}{l}0.00531 \\
(0.0162)\end{array}$ & $\begin{array}{l}-0.00348 \\
(0.0115)\end{array}$ \\
\hline Urban & $\begin{array}{c}0.0122 \\
(0.00876)\end{array}$ & $\begin{array}{c}0.00628 \\
(0.00663)\end{array}$ & $\begin{array}{c}0.00908 \\
(0.00908)\end{array}$ & $\begin{array}{c}0.00466 \\
(0.00667)\end{array}$ \\
\hline Nuclear Family & $\begin{array}{l}-0.0115^{*} \\
(0.00695)\end{array}$ & $\begin{array}{l}-0.00331 \\
(0.00512)\end{array}$ & $\begin{array}{l}-0.00931 \\
(0.00710)\end{array}$ & $\begin{array}{l}-0.00249 \\
(0.00511)\end{array}$ \\
\hline Children in $\mathrm{HH}$ & $\begin{array}{c}-0.0562^{* * *} \\
(0.0110)\end{array}$ & $\begin{array}{l}-0.0436^{* * *} \\
(0.00710)\end{array}$ & $\begin{array}{c}-0.0514^{* * *} \\
(0.0117)\end{array}$ & $\begin{array}{l}-0.0411^{* * *} \\
(0.00725)\end{array}$ \\
\hline Birth Interval & $\begin{array}{l}0.0736^{* * *} \\
(0.00673)\end{array}$ & $\begin{array}{l}0.0720^{* * *} \\
(0.00630)\end{array}$ & $\begin{array}{l}0.0739^{* * *} \\
(0.00652)\end{array}$ & $\begin{array}{l}0.0723^{* * *} \\
(0.00624)\end{array}$ \\
\hline $\begin{array}{l}\text { FE } \\
\text { p-value } \\
\text { Overid Test Stat } \\
\text { Observations }\end{array}$ & $\begin{array}{c}\text { no } \\
0.569 \\
9.613 \\
25511 \\
\end{array}$ & $\begin{array}{c}\text { yes } \\
0.221 \\
20.55 \\
25511\end{array}$ & $\begin{array}{c}\text { no } \\
\qquad \cdot \\
23.45 \\
25511\end{array}$ & $\begin{array}{c}\text { Yes } \\
\cdot \\
57.72 \\
25511\end{array}$ \\
\hline
\end{tabular}

Standard errors in parentheses

Columns 1 and 2: flood shocks across ages 12 to 13,14 to 15 and 16 to 17 are used as IVs. Column 3 and 4 use the average of flood shocks across the ages 12 to 17 as an IV.

${ }^{*} p<.1,{ }^{* *} p<.05,{ }^{* * *} p<.01$ 
Table 2.8: IV Results - Child Mortality by Gender

\begin{tabular}{|c|c|c|c|c|}
\hline & $\begin{array}{c}\text { (1) } \\
\text { male } \\
\text { deadbefore5 }\end{array}$ & $\begin{array}{c}(2) \\
\text { male } \\
\text { deadbefore } 5\end{array}$ & $\begin{array}{c}(3) \\
\text { female } \\
\text { deadbefore5 }\end{array}$ & $\begin{array}{c}(4) \\
\text { Female } \\
\text { deadbefore5 }\end{array}$ \\
\hline Child Marriage & $\begin{array}{l}1.418^{* * *} \\
(0.494)\end{array}$ & $\begin{array}{c}0.890^{* * *} \\
(0.268)\end{array}$ & $\begin{array}{l}1.382^{* *} \\
(0.687)\end{array}$ & $\begin{array}{l}0.621^{* *} \\
(0.298)\end{array}$ \\
\hline Age at Birth & $\begin{array}{l}0.0153^{* * *} \\
(0.00555)\end{array}$ & $\begin{array}{c}0.00877^{* * *} \\
(0.00297)\end{array}$ & $\begin{array}{c}0.0154^{*} \\
(0.00787)\end{array}$ & $\begin{array}{c}0.00594^{*} \\
(0.00325)\end{array}$ \\
\hline Highest Quintile & $\begin{array}{l}0.0931^{* *} \\
(0.0463)\end{array}$ & $\begin{array}{c}0.0360 \\
(0.0250)\end{array}$ & $\begin{array}{c}0.0537 \\
(0.0460)\end{array}$ & $\begin{array}{l}0.00191 \\
(0.0204)\end{array}$ \\
\hline Fourth Quintile & $\begin{array}{c}0.0119 \\
(0.0189)\end{array}$ & $\begin{array}{r}-0.00447 \\
(0.0136)\end{array}$ & $\begin{array}{l}0.00653 \\
(0.0194)\end{array}$ & $\begin{array}{c}-0.00708 \\
(0.0127)\end{array}$ \\
\hline Lowest Quintile & $\begin{array}{c}0.0665^{\text {*** }} \\
(0.0229)\end{array}$ & $\begin{array}{c}0.0443^{* * *} \\
(0.0154)\end{array}$ & $\begin{array}{c}0.0698^{* * *} \\
(0.0240)\end{array}$ & $\begin{array}{c}0.0479^{* * *} \\
(0.0140)\end{array}$ \\
\hline Second Quintile & $\begin{array}{c}0.0589^{* * *} \\
(0.0209)\end{array}$ & $\begin{array}{l}0.0474^{* * *} \\
(0.0150)\end{array}$ & $\begin{array}{c}0.0326^{*} \\
(0.0183)\end{array}$ & $\begin{array}{l}0.0299^{* *} \\
(0.0129)\end{array}$ \\
\hline Father-Primary Edu & $\begin{array}{l}0.0419^{* *} \\
(0.0193)\end{array}$ & $\begin{array}{l}0.0269^{* *} \\
(0.0126)\end{array}$ & $\begin{array}{c}0.0310 \\
(0.0285)\end{array}$ & $\begin{array}{l}0.00282 \\
(0.0145)\end{array}$ \\
\hline Mother-Primary Edu & $\begin{array}{l}0.0648^{*} \\
(0.0377)\end{array}$ & $\begin{array}{c}0.0362 \\
(0.0239)\end{array}$ & $\begin{array}{c}0.0569 \\
(0.0458)\end{array}$ & $\begin{array}{c}0.0130 \\
(0.0235)\end{array}$ \\
\hline Urban & $\begin{array}{c}0.0358^{*} \\
(0.0193)\end{array}$ & $\begin{array}{c}0.0215^{*} \\
(0.0130)\end{array}$ & $\begin{array}{c}0.0543 \\
(0.0374)\end{array}$ & $\begin{array}{c}0.0149 \\
(0.0180)\end{array}$ \\
\hline Nuclear Family & $\begin{array}{c}-0.0145 \\
(0.0135)\end{array}$ & $\begin{array}{r}-0.00129 \\
(0.0105)\end{array}$ & $\begin{array}{l}0.00152 \\
(0.0137)\end{array}$ & $\begin{array}{l}0.00517 \\
(0.0101)\end{array}$ \\
\hline Children in $\mathrm{HH}$ & $\begin{array}{c}-0.115^{* * *} \\
(0.0271)\end{array}$ & $\begin{array}{c}-0.0850^{* * *} \\
(0.0148)\end{array}$ & $\begin{array}{c}-0.112^{* * *} \\
(0.0374)\end{array}$ & $\begin{array}{c}-0.0695^{* * *} \\
(0.0162)\end{array}$ \\
\hline Birth Interval & $\begin{array}{c}0.0915^{* * *} \\
(0.0171)\end{array}$ & $\begin{array}{c}0.0901^{* * *} \\
(0.0134)\end{array}$ & $\begin{array}{c}0.0820^{* * *} \\
(0.0173)\end{array}$ & $\begin{array}{c}0.0772^{* * *} \\
(0.0126)\end{array}$ \\
\hline $\mathrm{FE}$ & no & yes & no & Yes \\
\hline p-value & 0.945 & 0.461 & 0.829 & 0.615 \\
\hline Overid Test Stat & 4.288 & 9.136 & 2.168 & 6.045 \\
\hline Observations & 10664 & 10664 & 10255 & 10255 \\
\hline
\end{tabular}

Standard errors in parentheses

Columns 1 to 4 : flood shocks across ages 12 to 13,14 to 15 and 16 to 17 are used as IVs. ${ }^{*} p<.1,{ }^{* *} p<.05,{ }^{* * *} p<.01$ 
Table 2.9: IV Results - Infant Mortality by Gender

\begin{tabular}{|c|c|c|c|c|}
\hline & $\begin{array}{c}\text { (1) } \\
\text { deadbefore1 }\end{array}$ & $\begin{array}{c}(2) \\
\text { deadbefore1 }\end{array}$ & $\begin{array}{c}\text { (3) } \\
\text { deadbefore1 }\end{array}$ & $\begin{array}{c}\text { (4) } \\
\text { deadbefore1 }\end{array}$ \\
\hline Child Marriage & $\begin{array}{l}0.696^{* *} \\
(0.283)\end{array}$ & $\begin{array}{l}0.377^{* *} \\
(0.172)\end{array}$ & $\begin{array}{c}0.370 \\
(0.234)\end{array}$ & $\begin{array}{c}0.227 \\
(0.159)\end{array}$ \\
\hline Age at Birth & $\begin{array}{l}0.00809^{* *} \\
(0.00338)\end{array}$ & $\begin{array}{c}0.00389^{*} \\
(0.00202)\end{array}$ & $\begin{array}{c}0.00463 \\
(0.00311)\end{array}$ & $\begin{array}{c}0.00235 \\
(0.00203)\end{array}$ \\
\hline Highest Quintile & $\begin{array}{c}0.0441 \\
(0.0281)\end{array}$ & $\begin{array}{l}0.00781 \\
(0.0171)\end{array}$ & $\begin{array}{l}0.00874 \\
(0.0173)\end{array}$ & $\begin{array}{l}-0.00404 \\
(0.0123)\end{array}$ \\
\hline Fourth Quintile & $\begin{array}{c}0.000929 \\
(0.0117)\end{array}$ & $\begin{array}{l}-0.00890 \\
(0.00922)\end{array}$ & $\begin{array}{l}-0.00544 \\
(0.00891)\end{array}$ & $\begin{array}{l}-0.00738 \\
(0.00847)\end{array}$ \\
\hline Lowest Quintile & $\begin{array}{l}0.0307^{* *} \\
(0.0140)\end{array}$ & $\begin{array}{c}0.0160 \\
(0.0103)\end{array}$ & $\begin{array}{c}0.0161^{*} \\
(0.00953)\end{array}$ & $\begin{array}{c}0.0113 \\
(0.00875)\end{array}$ \\
\hline Second Quintile & $\begin{array}{l}0.0305^{* *} \\
(0.0130)\end{array}$ & $\begin{array}{l}0.0223^{* *} \\
(0.0102)\end{array}$ & $\begin{array}{c}0.00922 \\
(0.00908)\end{array}$ & $\begin{array}{c}0.0106 \\
(0.00856)\end{array}$ \\
\hline Father-Primary Edu & $\begin{array}{l}0.0259^{* *} \\
(0.0130)\end{array}$ & $\begin{array}{c}0.0142 \\
(0.00913)\end{array}$ & $\begin{array}{c}0.000716 \\
(0.0107)\end{array}$ & $\begin{array}{l}-0.00389 \\
(0.00862)\end{array}$ \\
\hline Mother-Primary Edu & $\begin{array}{c}0.0306 \\
(0.0241)\end{array}$ & $\begin{array}{c}0.0107 \\
(0.0168)\end{array}$ & $\begin{array}{l}0.00747 \\
(0.0193)\end{array}$ & $\begin{array}{c}-0.000414 \\
(0.0151)\end{array}$ \\
\hline Urban & $\begin{array}{c}0.0187 \\
(0.0118)\end{array}$ & $\begin{array}{c}0.0116 \\
(0.00887)\end{array}$ & $\begin{array}{l}0.00968 \\
(0.0132)\end{array}$ & $\begin{array}{l}0.00385 \\
(0.0101)\end{array}$ \\
\hline Nuclear Family & $\begin{array}{c}-0.0287^{* *} \\
(0.0112)\end{array}$ & $\begin{array}{c}-0.0150^{*} \\
(0.00763)\end{array}$ & $\begin{array}{c}0.00124 \\
(0.00890)\end{array}$ & $\begin{array}{c}0.00689 \\
(0.00706)\end{array}$ \\
\hline Children in $\mathrm{HH}$ & $\begin{array}{c}-0.0716^{* * *} \\
(0.0165)\end{array}$ & $\begin{array}{c}-0.0521^{* * *} \\
(0.0102)\end{array}$ & $\begin{array}{c}-0.0509^{* * *} \\
(0.0144)\end{array}$ & $\begin{array}{l}-0.0413^{* * *} \\
(0.00981)\end{array}$ \\
\hline Birth Interval & $\begin{array}{c}0.0700^{* * *} \\
(0.0109)\end{array}$ & $\begin{array}{l}0.0695^{* * *} \\
(0.00937)\end{array}$ & $\begin{array}{l}0.0754^{* * *} \\
(0.00913)\end{array}$ & $\begin{array}{l}0.0733^{* * *} \\
(0.00874)\end{array}$ \\
\hline $\mathrm{FE}$ & no & yes & no & Yes \\
\hline p-value & 0.501 & 0.0877 & 0.374 & 0.242 \\
\hline Overid Test Stat & 5.362 & 10.85 & 5.326 & 10.64 \\
\hline Observations & 13017 & 13017 & 12494 & 12494 \\
\hline
\end{tabular}

Standard errors in parentheses

Columns 1 to 4 : flood shocks across ages 12 to 13,14 to 15 and 16 to 17 are used as IVs. ${ }^{*} p<.1,{ }^{* *} p<.05,{ }^{* * *} p<.01$ 
Table 2.10: IV Results - Access to Healthcare

\begin{tabular}{|c|c|c|c|c|}
\hline & $\begin{array}{c}\text { (1) } \\
\text { Go Outside } \\
\text { Alone } \\
\end{array}$ & $\begin{array}{c}\text { (2) } \\
\text { Go to Hospital } \\
\text { Alone } \\
\end{array}$ & $\begin{array}{l}\text { (3) } \\
\text { Permission to go to } \\
\text { Health Facility }\end{array}$ & $\begin{array}{c}\text { (4) } \\
\text { Money for } \\
\text { Treatment } \\
\end{array}$ \\
\hline $\mathrm{CM}$ & $\begin{array}{c}0.233 \\
(0.225)\end{array}$ & $\begin{array}{l}-0.533^{*} \\
(0.281)\end{array}$ & $\begin{array}{l}0.510^{* * *} \\
(0.190)\end{array}$ & $\begin{array}{c}0.00916 \\
(0.143)\end{array}$ \\
\hline Fourth Q & $\begin{array}{c}0.00461 \\
(0.0172)\end{array}$ & $\begin{array}{c}0.0311 \\
(0.0338)\end{array}$ & $\begin{array}{r}-0.00941 \\
(0.0188)\end{array}$ & $\begin{array}{c}-0.115^{* * *} \\
(0.0142)\end{array}$ \\
\hline Highest Q & $\begin{array}{c}0.0474 \\
(0.0323)\end{array}$ & $\begin{array}{c}0.0281 \\
(0.0506)\end{array}$ & $\begin{array}{c}-0.000647 \\
(0.0310)\end{array}$ & $\begin{array}{l}-0.233^{* * *} \\
(0.0233)\end{array}$ \\
\hline Lowest Q & $\begin{array}{l}0.0417^{* *} \\
(0.0175)\end{array}$ & $\begin{array}{l}-0.0111 \\
(0.0328)\end{array}$ & $\begin{array}{c}0.0279 \\
(0.0186)\end{array}$ & $\begin{array}{c}0.0807^{* * *} \\
(0.0140)\end{array}$ \\
\hline Second Q & $\begin{array}{c}0.0211 \\
(0.0165)\end{array}$ & $\begin{array}{l}-0.0155 \\
(0.0325)\end{array}$ & $\begin{array}{c}0.0321^{*} \\
(0.0177)\end{array}$ & $\begin{array}{c}0.0511^{* * *} \\
(0.0134)\end{array}$ \\
\hline Mother- Edu & $\begin{array}{l}0.0473^{* *} \\
(0.0217)\end{array}$ & $\begin{array}{c}0.00190 \\
(0.0328)\end{array}$ & $\begin{array}{c}-0.00593 \\
(0.0200)\end{array}$ & $\begin{array}{c}-0.0851^{* * *} \\
(0.0151)\end{array}$ \\
\hline Father-Edu & $\begin{array}{c}-0.0158 \\
(0.0141)\end{array}$ & $\begin{array}{l}0.00177 \\
(0.0266)\end{array}$ & $\begin{array}{c}0.00303 \\
(0.0155)\end{array}$ & $\begin{array}{c}-0.0379^{* * *} \\
(0.0117)\end{array}$ \\
\hline Age & $\begin{array}{l}0.00403^{* * *} \\
(0.000597)\end{array}$ & $\begin{array}{c}0.00194^{*} \\
(0.00109)\end{array}$ & $\begin{array}{c}-0.00224^{* * *} \\
(0.000622)\end{array}$ & $\begin{array}{l}0.00186^{* * *} \\
(0.000469)\end{array}$ \\
\hline Urban & $\begin{array}{c}0.0632^{* * *} \\
(0.0149)\end{array}$ & $\begin{array}{c}0.0262 \\
(0.0290) \\
\end{array}$ & $\begin{array}{c}-0.0668^{* * *} \\
(0.0151) \\
\end{array}$ & $\begin{array}{c}0.0107 \\
(0.0114) \\
\end{array}$ \\
\hline $\begin{array}{l}\text { FE } \\
\text { p-value } \\
\text { Overid Test } \\
\text { Observations }\end{array}$ & $\begin{array}{c}\text { yes } \\
0.493 \\
10.40 \\
7285\end{array}$ & $\begin{array}{c}\text { yes } \\
0.750 \\
7.927 \\
1998\end{array}$ & $\begin{array}{c}\text { yes } \\
0.125 \\
20.04 \\
9299\end{array}$ & $\begin{array}{c}\text { yes } \\
0.852 \\
20.10 \\
9300\end{array}$ \\
\hline
\end{tabular}

Standard errors in parentheses

Columns 1 to 4 : flood shocks across ages 12 to 13,14 to 15 and 16 to 17 are used as IVs.

${ }^{*} p<.1,{ }^{* *} p<.05,{ }^{* * *} p<.01$ 
Table 2.11: IV Results -Final Say Decisions

\begin{tabular}{|c|c|c|c|c|c|c|}
\hline & $\begin{array}{c}(1) \\
\text { Own } \\
\text { Health } \\
\text { Care }\end{array}$ & $\begin{array}{c}(2) \\
\text { Child } \\
\text { Health } \\
\text { Care }\end{array}$ & $\begin{array}{c}\text { (3) } \\
\text { Large HH } \\
\text { purchases }\end{array}$ & $\begin{array}{c}\text { (4) } \\
\text { Daily HH } \\
\text { Purchases }\end{array}$ & $\begin{array}{c}(5) \\
\text { Visits }\end{array}$ & $\begin{array}{c}(6) \\
\text { Food } \\
\text { Cooked } \\
\text { Daily }\end{array}$ \\
\hline $\mathrm{CM}$ & $\begin{array}{c}-0.366^{* *} \\
(0.152)\end{array}$ & $\begin{array}{l}-0.354^{* *} \\
(0.147)\end{array}$ & $\begin{array}{c}0.00946 \\
(0.110)\end{array}$ & $\begin{array}{l}-0.278^{*} \\
(0.146)\end{array}$ & $\begin{array}{c}-0.0913 \\
(0.126)\end{array}$ & $\begin{array}{c}-0.877^{* * *} \\
(0.205)\end{array}$ \\
\hline Fourth Q & $\begin{array}{l}-0.0184 \\
(0.0150)\end{array}$ & $\begin{array}{l}-0.0287^{*} \\
(0.0154)\end{array}$ & $\begin{array}{c}-0.00726 \\
(0.0109)\end{array}$ & $\begin{array}{l}-0.0166 \\
(0.0144)\end{array}$ & $\begin{array}{l}-0.00851 \\
(0.0124)\end{array}$ & $\begin{array}{c}-0.0814^{* * *} \\
(0.0203)\end{array}$ \\
\hline Highest Q & $\begin{array}{l}-0.0181 \\
(0.0253)\end{array}$ & $\begin{array}{l}-0.0251 \\
(0.0245)\end{array}$ & $\begin{array}{l}0.00139 \\
(0.0184)\end{array}$ & $\begin{array}{l}-0.0429^{*} \\
(0.0243)\end{array}$ & $\begin{array}{l}-0.0131 \\
(0.0209)\end{array}$ & $\begin{array}{l}-0.193^{* * *} \\
(0.0343)\end{array}$ \\
\hline Lowest Q & $\begin{array}{c}0.0106 \\
(0.0145)\end{array}$ & $\begin{array}{c}0.0152 \\
(0.0146)\end{array}$ & $\begin{array}{c}0.0347^{* * *} \\
(0.0105)\end{array}$ & $\begin{array}{c}0.0158 \\
(0.0139)\end{array}$ & $\begin{array}{l}0.0287^{* *} \\
(0.0120)\end{array}$ & $\begin{array}{c}0.0792^{* * *} \\
(0.0196)\end{array}$ \\
\hline Second Q & $\begin{array}{c}-0.00734 \\
(0.0139)\end{array}$ & $\begin{array}{c}-0.00176 \\
(0.0140)\end{array}$ & $\begin{array}{l}0.00848 \\
(0.0100)\end{array}$ & $\begin{array}{c}-0.000815 \\
(0.0133)\end{array}$ & $\begin{array}{c}0.000449 \\
(0.0114)\end{array}$ & $\begin{array}{c}0.0358^{*} \\
(0.0187)\end{array}$ \\
\hline Mother-Edu & $\begin{array}{c}-0.0547^{* * *} \\
(0.0160)\end{array}$ & $\begin{array}{c}-0.0327^{* *} \\
(0.0157)\end{array}$ & $\begin{array}{c}-0.00509 \\
(0.0116)\end{array}$ & $\begin{array}{r}-0.00695 \\
(0.0154)\end{array}$ & $\begin{array}{r}-0.00957 \\
(0.0132)\end{array}$ & $\begin{array}{c}-0.0598^{* * *} \\
(0.0217)\end{array}$ \\
\hline Father-Edu & $\begin{array}{c}-0.0330^{* * *} \\
(0.0123)\end{array}$ & $\begin{array}{l}-0.0203^{*} \\
(0.0122)\end{array}$ & $\begin{array}{c}0.000795 \\
(0.00892)\end{array}$ & $\begin{array}{l}0.00141 \\
(0.0119)\end{array}$ & $\begin{array}{r}-0.00171 \\
(0.0102)\end{array}$ & $\begin{array}{c}-0.0430^{* * *} \\
(0.0167)\end{array}$ \\
\hline Age & $\begin{array}{l}0.00635^{* * *} \\
(0.000471)\end{array}$ & $\begin{array}{c}0.00689^{* * *} \\
(0.000478)\end{array}$ & $\begin{array}{c}0.00505^{* * *} \\
(0.000340)\end{array}$ & $\begin{array}{c}0.00626^{* * *} \\
(0.000452)\end{array}$ & $\begin{array}{c}0.00691^{* * *} \\
(0.000388)\end{array}$ & $\begin{array}{c}0.0117^{\text {*** }} \\
(0.000637)\end{array}$ \\
\hline Urban & $\begin{array}{l}0.0436^{* * *} \\
(0.0122)\end{array}$ & $\begin{array}{c}0.0417^{* * *} \\
(0.0121)\end{array}$ & $\begin{array}{c}0.0649^{* * *} \\
(0.00880)\end{array}$ & $\begin{array}{c}0.0768^{* * *} \\
(0.0117)\end{array}$ & $\begin{array}{c}0.0510^{* * *} \\
(0.0100)\end{array}$ & $\begin{array}{c}0.0254 \\
(0.0165)\end{array}$ \\
\hline $\begin{array}{l}\text { FE } \\
\text { p-value }\end{array}$ & yes & yes & yes & yes & Yes & yes \\
\hline Overid Test & 60.58 & 63.66 & 60.40 & 60.52 & 59.99 & 60.69 \\
\hline Observations & 10330 & 9924 & 10325 & 10321 & 10324 & 10327 \\
\hline
\end{tabular}

Standard errors in parentheses

Columns 1 to 4 : the average of flood shocks across ages 12 to 17 is used as an IV.

${ }^{*} p<.1,{ }^{* *} p<.05,{ }^{* * *} p<.01$ 


\section{CHAPTER 3}

\section{SHARIA LAW, CHILDREN'S HEALTH OUTCOMES AND WOMEN'S EMPOWERMENT: DATA FROM NIGERIA}

\subsection{Introduction}

Malnutrition is unquestionably the largest impediment to child survival and growth. Worldwide, nearly half of all deaths in children under five are linked to malnutrition (Black et al. 2013). In Nigeria, malnutrition among children remains a significant problem; onethird of children under five are stunted and one-fifth of children are underweight. Malnourished children are more vulnerable to infection, and face an increased risk of death from diarrhea, pneumonia and measles. For the children that survive, the impact of malnutrition continues to affect them throughout their life (Forouzanfar et al. 2015). Stunted growth in children is associated with delays in cognitive development and decreases in educational achievement; in later life, stunting is linked with a reduction in productivity and lower lifetime earnings (Kar et al. 2008, Martorell et al. 2008, Walker et al. 2007).

Few studies in the economic literature have acknowledged the influence of religious rules and customs on child health; Bhalotra et al. (2010) analyze the differences in child survival amongst Muslims and high-caste Hindus in India and find that the survival of female children is higher among Muslims. In this paper, I contribute to the empirical literature on the relationship between religion and child health by examining the effect of a religion differential on children's outcomes. Specifically, this paper identifies the effect 
of Sharia Law in Nigeria on children's nutritional outcomes, by using the anthropometric measures of stunting, wasting and underweight.

The identification strategy exploits the change in the Nigerian Legal System in 1999, in which 12 of the 36 states enacted a reform of their legal system, by introducing Sharia Law to encompass criminal and non-criminal matters (Ostien 2007). The introduction of the reform led to corporal punishments (amputations and stoning) being instituted for crimes, and reduced the legal rights of women (Iman 2003). To assess the impact of Sharia Law on children's health outcomes, I employ a difference-in-differences framework and make use of the variation in exposure to Sharia Law across time and state of residence. The model is estimated using a unique dataset that merges information on child health outcomes from the Nigerian Demographic Health Surveys in 1990 and 2003.

Also of interest when examining the topic of child health, is the empowerment of women in Nigeria. Women's empowerment is a major determinant of child growth and survival. As primary caregivers, women can directly and indirectly impact the nutritional outcomes of their children through their own nutritional status, prenatal care usage, feeding practices and treatment of illnesses (Smith et al. 2003). However, the extent to which mothers can affect child health is dependent on empowerment. Mothers who are more empowered have increased control of resources, greater relative decision-making power, more access to information and health services and higher mobility (Ghulam et al. 2006, Luz and Agadjanian 2015). These factors are closely related to the mother's nutritional status, and in turn, the quality of care her children receive. As a supplementary analysis, I explore a possible channel connecting Sharia Law to children's health outcomes by investigating the effect of Sharia Law on the empowerment of mothers. The adoption of 
Sharia Law disproportionally affects women and has the potential to impact empowerment; physical mobility is restricted as women are not allowed to travel in the same vehicles as men, and control over resources is limited as women are banned from renting houses. Lamidi (2006) uses data in Nigeria after the implementation of Sharia Law and finds significant variations in women's participation in household decision-making. Further, the results show that women who live in states with Sharia Law have less decision-making power than women in states without Sharia Law.

The main finding to emerge from the estimations is that there is a statistically significant increase in stunted children in states in which the Sharia Law was implemented, relative to all other children. Numerically, the adoption of Sharia Law increased the probability of stunting by 8.80 percentage points. To assess whether the increase represents moderate or severe stunting, I explore the effect on the differences in the levels of severity of malnutrition. I find an increase of 5.15 percentage points in severe stunting; the estimate on moderate stunting is small in magnitude and not statistically significant. The results also indicate a small decrease in wasting, but validity of the estimate remains questionable as the F-statistic value is very low. Additionally, the decrease in wasting may be a sideeffect of the increase in stunting.

Anecdotal evidence shows that although Sharia Law was implemented in all 12 states, three states in particular had a higher level of enforcement. I extend the analysis to investigate whether Sharia Law has a larger impact on children's outcomes in states with high implementation. The findings highlight that children exposed to Sharia Law in high implementation states experienced a 20.2 percentage point increase in stunting and an 11.1 percentage point increase in being underweight, relative to all other children. A further 
analysis on severity levels indicates that the effect is concentrated on increases in severe stunting and being severely underweight.

The findings from the supplementary analysis suggest a strong negative association between Sharia Law and women's empowerment. The results reveal that mothers residing in states with Sharia Law are less likely to participate in household decision-making, less able to make health care decisions for themselves and their children and less likely to have financial autonomy.

The remainder of the paper is organized as follows: Section 3.2 provides a background of Sharia Law in Nigeria. Section 3.3 details the data used and provides descriptive statistics. The empirical strategy is discussed in Section 3.4. Section 3.5 provides the main empirical results and Section 3.6 provides the details on the supplementary analysis. Section 3.7 concludes the paper.

\subsection{Sharia Law in Nigeria}

In 1960, Nigeria gained independence after nearly 100 years under British colonization. The new Nigerian Legal System was heavily influenced by English Common Law, but also incorporated Customary Law for personal status matters including marriage, divorce, inheritance, guardianship and custody of children. Customary law was divided into Ethnic Law and Islamic (Sharia) Law. Ethnic customary law is an indigenous law that takes into consideration the culture, customs and values of the different ethnic groups in Nigeria (Dina et al. 2005). The foundation for Islamic Law is the Holy Quran and it is modelled after the teachings of the Prophet Muhammed. The legal system allowed non-criminal 
matters to fall under the jurisdiction of Islamic courts and was kept in place until 1999 (Tertsakian 2004).

In $1999,12^{25}$ of the 36 states in Nigeria enacted a reform of their legal system by introducing Islamic Law as the dominant legal system (Ostien 2007). Essentially, the reform instituted Islamic Law as the main body of law for criminal matters in these 12 states. Prior to 1999, the scope of Islamic Law courts was limited to only personal status matters and civil law.

Under Sharia Law, criminal offences that were once punishable by imprisonment and fines, are now punishable by amputation of limbs, flogging and stoning (Peters 2005). The reform reduces the litigation rights of women against sexual abuse and violence, and puts them at a disadvantage relative to men (Iman 2003). For example, under Sharia Law husbands cannot be charged for marital rape, and provisions are made for wife-beating under the condition that grievous bodily harm is not inflicted (Ekhator 2005).

Anecdotal evidence shows that the adoption of Sharia Law affects women's autonomy. To illustrate, the mobility of women is restricted as they are not allowed to travel in the same vehicles as men, and women's control over assets is limited as they are prohibited from renting houses. Individual choice is also impacted through constraints placed on female clothing choices; nurses must wear hijabs and trousers as a part of their uniform or face dismissal, high-school students are required to wear hijabs, and all Western forms of dress are banned. In addition, the reform decreases access to employment opportunities as women are barred from becoming lawyers or judges (Adejumo and Fwacn 2011). One

\footnotetext{
25 The 12 states include: Zamfara, Kano, Sokoto, Katsina, Bauchi, Borno, Jigawa, Kebbi, Yobe, Kaduna, Niger and Gombe.
} 
study has examined the state variations in women's participation in household decisionmaking after the implementation of Sharia Law. The results of the study show lower decision-making power among women who live in states that have adopted Sharia Law, in comparison to women who live in non-reform states (Lamidi 2016).

\subsection{Data}

For the analysis, I use data from the 1990 and 2003 Nigeria Demographic and Health Survey (NDHS). The NDHS is a nationally representative study conducted by the National Population Council and collected using a stratified, three-stage cluster sampling strategy. The first data collection phase of the NDHS was conducted in 1990, in which 8,781 women between the ages of 15 to 49 were interviewed from 8,999 households. In the 2003 NDHS, 7,620 women between the ages of 15 to 49 and 2,346 men between the ages of 15 and 59 from 7,225 households were interviewed. The survey provides information on housing characteristics, background characteristics, marriage and sexual activity, fertility preferences, decision-making, childhood mortality, adult mortality and family planning methods. For each interviewed woman, the data relating to prenatal and postnatal care, immunization coverage, and health indicators of children born in the five years preceding the survey is collected.

\subsubsection{Child Outcomes}

I measure children's health outcomes by nutritional status. To determine nutritional status, three anthropometric indices, specifically height-for-age, weight-for-height and weightfor-age are used. Children are classified as stunted if their height-for-age is more than 2 
standard deviations below the World Health Organization Child Growth Standards median (WHO 2006). Stunting reflects the effect of long-term nutritional deprivation and results in adverse health and development consequences for the individual across childhood and adulthood (Dewey and Begum 2011). Several studies show that stunting is associated with poorer cognitive development, diminished learning capacity and lower educational achievement (Kar et al. 2008, Martorell et al. 2008, Walker et al. 2007). Childhood stunting is also a risk factor for mortality and chronic diseases in adulthood and permanent cognitive impairment (Huffman and Schofield 2011). Additionally, childhood stunting is linked with a reduction in human capital and economic productivity in adulthood, leading to lower earnings (Kar et al. 2008, Thomas and Strauss 2011). The weight-for-height index is used to identify wasting. Wasting occurs in children whose weight-for-height is more than 2 standard deviations below the World Health Organization Child Growth Standards median (WHO 2006). Wasting represents the loss of weight or failure to gain weight, and is usually the result of inadequate caring and feeding practices, poor food security or a recent episode of illness (for example, diarrheal disease) (UNICEF 2007, Bloss et al. 2004). Children who are wasted are more susceptible to infections and face a heightened risk of mortality (Black et al. 2008).

The weight-for-age index is a composite index of both height-for-age and weight-forheight. Children whose weight-for-age is more than 2 standard deviations below the World Health Organization Child Growth Standards median are considered underweight (WHO 2006). Due to the missing height dimension, the interpretation of nutritional status using weight-for-age may be complicated as it fails to distinguish between children who are low in weight relative to their height (tall, thin child) and children who are low in height relative 
to their age (short child with adequate weight) but who may be classified as normal using the weight-for-height index (Bose et al. 2007, WHO 2010).

\subsubsection{Final Sample}

I limit the sample to children between the ages of 0 and 5, as data relating to children's prenatal and postnatal care, immunization status, nutritional status and other health indicators are only collected for children born in the five years preceding the survey. Additionally, the sample only includes living children, due to missing data on anthropometry measures of all dead children. To address possible confounding factors, I further restrict the sample to children from Muslim-majority states, including: Sokoto, Zamfara, Katsina, Jigawa, Yobe, Borno, Adamawa, Gombe, Bauchi, Kano, Kaduna, Kebbi, Niger, Abuja, Nasarawa, Kogi, Kwara, Oyo, Osun, Ondo, Lagos and Odun. The inclusion of all children may lead to biased results as Muslim-majority states may differ intrinsically from other states. The final sample consists of 6,427 children's cases: 3,478 cases from 1990 and 2,949 cases from 2003.

In Table 3.1 I present summary statistics for children in the sample. Children in 2003 are from poorer households than children in 1990; on average, 41.5 percent of children in 2003 are from households below the middle quintile, while 36 percent of children are in 1990. Nearly half of the children in 1990 live in urban areas, while in 2003, the majority of children are in rural areas. The mean number of members in the household is relatively high in both years, at approximately seven. In regard to parental education, fathers in general are more educated than mothers and, compared to parents in 1990, parents in 2003 have attained more education. On average, 56.4 percent of fathers and 42.4 percent of 
mothers in 2003 have a primary education or higher, while in 1990, 47.4 percent of fathers and 36.8 percent of mothers have a primary education or higher. Birth characteristics show that the mother's average age at birth in both years is 26 years, and that approximately 6 to 7 percent of children are born within 18 months of the previous birth. Additionally, the average birth order is 3.835 in 1990 and 4.038 in 2003, indicating that a large number of children are thirdborn. The sample in both years is evenly split between male and female children.

The anthropometry measures indicate that the levels of stunting and being underweight in children decreased from 1990 to 2003. In contrast, the levels of wasting increased from 10.7 percent in 1990 to 12.4 percent in 2003. Regarding the states in which the reform was enacted, more than half of the children reside in these states, although the number of children in 2003 (70.8 percent) is higher than the number of children in 1990 (52.4 percent). A relatively small number of children live in states in which the reform was highly implemented; approximately 18 percent of children from the sample in 1990 reside in high implementation states, while in 2003, 13 percent of the children reside in high implementation states.

\subsection{Empirical Strategy}

I exploit the change in the Nigerian legal system in 1999, in which 12 states adopted Sharia Law. In order to investigate the effect of Sharia Law on children's health outcomes, I apply a difference-in-differences framework and make use of the variation in exposure to Sharia Law across time and state of residence. 
The empirical strategy involves estimating the following model:

$Y_{i, t}=\alpha_{0}+\alpha_{1}$ After $_{t}+\alpha_{2}$ Sharia $_{i}+\alpha_{3}$ After $_{t}$ XSharia $_{i}+\alpha_{4} X_{i, t}+\delta_{s}+\mu_{i, s, t}$

where $Y_{i, t}$ is the health outcome of child $i$. Dichotomous indicators are constructed for each outcome: stunting, underweight and wasting. In accordance with the World Health Organization Child Growth Standards criterion, each indicator takes the value of 1 if the child's height-for-age, weight-for-height and weight-for-age index separately is more than 2 standard deviations below the reference median (WHO 2006), and 0 otherwise. After ${ }_{t}$ is a binary variable that is equal to 1 if $t>1999$ (time era following the implementation of Islamic Law, and 0 otherwise (time era prior to the implementation). Sharia $i$ is the treatment variable, which is assigned a value of 1 if child $i$ lives in one of the 12 states that enacted Sharia Law (treatment group), and 0 otherwise (control group).

$X_{i, t}$ is a vector of child, parental and household characteristics. Household characteristics include the wealth level of the household, the number of members within the household and the location (urban or rural). Using the wealth index ${ }^{26}$, four dummy variables are constructed to indicate the wealth level of the household: poorest quintile, poorer quintile, richer quintile and richest quintile. A dummy variable is constructed for location, and assigned a value of 1 if the household is in an urban area. Parental characteristics are represented by the educational level of the mother and father and the age at birth of the mother. The educational levels of the mother and father are each denoted by a dummy variable that is equal to 1 if the highest level of education achieved is primary

\footnotetext{
${ }^{26}$ The wealth index is a composite measure of the economic status of the household. The wealth index is calculated using data on household assets (ownership of televisions, bicycles), types of water access, sanitation facilities, availability of electricity and housing facilities.
} 
school or higher, and 0 otherwise. Child characteristics include birth order, gender and birth interval. Binary indicators are constructed for gender and birth interval, where a value of 1 is assigned if the child is female and if the preceding birth interval is within 18 months. Short birth intervals adversely affect child survival; Fotso et al. (2013) find that compared to children born after an interval of 36 months, children with a preceding birth interval of less than 18 months are twice as likely to die. Similarly, Becher et al. (2004) report that a preceding birth interval of less than 18 months is associated with an increase in the risk for infant mortality.

State fixed effects $\left(\delta_{s}\right)$ are included to control for unobservable characteristics in the state. The main coefficient of interest is $\alpha_{3}$, which measures the expected mean change in health outcomes from before to after for children in reform states, relative to other children.

To assess the levels of severity for each outcome, I re-estimate Equation (3.1) and substitute stunting, wasting, and underweight with indicator variables for moderate stunting, severe stunting, moderate underweight, severe underweight, moderate wasting and severe wasting. Children are considered to be moderately stunted (underweight, wasted) if their height-for-age (weight-for-age, weight-for-height) is between -2 standard deviations and -3 standard deviations from the Child Growth Standards median. Children are defined as severely stunted (underweight, wasted) if their height-for-age (weight-forage, weight-for-height) is more than 3 standard deviations below the Child Growth Standards median. Using these definitions, I construct indicator variables for each, where a value of 1 is assigned if child $i$ is moderately stunted (underweight, wasted) or severely stunted (underweight, wasted), and 0 otherwise. 


\subsection{Results}

\subsubsection{Main Results}

Table 3.2 reports the results of the difference-in-differences specification outlined in Equation 3.1 separately for each measure of children's nutritional status. The dependent variable is stunting in columns 1 and 4, underweight in columns 2 and 5, and wasting in columns 3 and 6, without and with the application of state fixed effects. Standard errors are presented in parentheses, and the number of observations, F-statistic and R-squared value are reported in the last three rows of the table.

The coefficient on the After variable reflects the effect of the passage of time in the absence of the enactment of Sharia Law. In comparison to children in the pre-era of implementation of Sharia Law (year 1990), children in the post-era of implementation of Sharia Law (year 2003) are less likely to be stunted and underweight. In contrast, children are more likely to be wasted. Without fixed effects, the results show that the probability of stunting in children decreases by 9.03 percentage points and the probability of being underweight declines by 4.20 percentage points, while there is an increase in the probability of wasting by 3.52 percentage points. The coefficient on the Reform variable represents the baseline differences that exist between the treatment and control group before Sharia Law was applied to the control group. The estimated mean difference in stunting between the treatment and control group prior to the enactment of Sharia Law is small in magnitude and not significantly different from zero. However, there is a difference between the two groups in underweight and wasting - children residing in reform states, relative to children 
in non-reform states, are 11.3 percentage points more likely to be underweight and 7.33 more likely to be wasted.

The results from the different-in-differences estimator indicate that children exposed to Sharia Law have experienced a significant increase in the probability of stunting after the reform was adopted, relative to all other children. The difference-in-differences estimate shows an increase of 8.80 percentage points in stunting, and is statistically significant at the 1 percent level. With fixed effects, the effect on stunting is relatively the same in magnitude and remains statistically significant at the 1 percent level. The Fstatistic, in both specifications with and without fixed effects, is large. The difference-indifference estimates report a decrease in wasting for children living in reform-states relative to other children. The validity of this estimate is questionable owing to the small F-statistic, and the decrease in statistical significance when fixed effects are employed. Additionally, the decrease in wasting may be a side-effect of the reported increase in stunting. Stunting is defined as low height-for-age, while wasting is defined as low weightfor-height; if stunting in children is increasing, this indicates that height is falling. As height declines, the weight-for-height index increases and results in less wasting in children. The results of the estimation also highlight important determinants of children's nutritional outcomes. The coefficients on age at birth, richer and richest wealth levels, female, paternal education are significantly and negatively correlated with the probability of stunting and being underweight, while the coefficients on birth order and poorest wealth levels are statistically significant and positively correlated. The direction of these correlations is in line with earlier studies on malnutrition (Bloss et al. 2004, Veghari 2007, Ahmed and Islam 1984). To illustrate, Egata et al. (2014), Vitolo et al. (2008) and Bairagi and Chowdhury 
(1994) find that the socioeconomic status of the household significantly affects children's nutritional status; poor households are more likely to be undernourished than households with higher wealth levels. Further, Rayhan and Khan (2006) find that children whose fathers had a primary and secondary education had a lower risk of being stunted and underweight in comparison to children of illiterate fathers. Additionally, Hien and Kam (2008) assess nutritional characteristics related to children under five and report that the odds of being underweight and stunted are lower among female children than among male children.

\subsubsection{Moderate and Severe Levels of Undernutrition}

To explore the effect of Sharia Law on differences in the levels of severity on malnutrition outcomes, I analyze whether children are moderately or severely stunted, underweight and wasted. The results are presented in Table 3.3. The difference-in-differences estimates, which are robust to the inclusion of controls, show an increase of 5.15 percentage points in severe stunting and a decrease of 2.55 percentage points in severe wasting. However, the validity of the effect on severe wasting remains questionable as the F-statistic is extremely low at 3.7. The estimates on moderate stunting, moderate wasting, moderate and severe underweight are small in magnitude and not statistically different from zero. The results suggest that the reform impacts children's nutritional outcomes through stunting, and is concentrated on increases in severe stunting. The reform does not seem to influence children being underweight as there is no visible change in the underweight estimates among children who were living in states that enacted Sharia Law. 


\subsubsection{High Implementation of Sharia Law}

Anecdotal evidence shows that although Sharia Law was enacted in 12 states, some states ${ }^{27}$ had a higher level of implementation than others. In particular, Weimann (2010) finds that cases in Sokoto, Zamfara and Katsina accounted for more than 60 percent of the total cases under the new Islamic legal system. To assess whether Sharia Law has a larger impact on children's outcomes in states with high implementation, I create an indicator variable that is assigned a value of 1 if the child resides in Sokoto, Zamfara or Katsina, and 0 otherwise, and subsequently construct a new interaction term (After X High ). I re-estimate Equation 3.1 using these new variables to examine the changes in nutritional outcomes among children that were exposed to Sharia Law and reside in states with high implementation. Table 3.4 presents the results, with and without fixed effects. The difference-in-differences estimates with fixed effects show that there is a statistically significant increase in the probability of stunting and being underweight for children in states with high implementation of Sharia Law, relative to all other children. The results indicate that children exposed to Sharia Law in high implementation states have experienced a 20.2 percentage point increase in stunting and an 11.1 percentage point increase in being underweight after the Law was enacted, relative to all other children. The impact on wasting in children remains unclear; the F-statistic in both specifications in Columns 3 and 6 is very low and the difference-in-differences estimator with fixed effects (Column 6) becomes smaller in magnitude and loses statistical significance.

\footnotetext{
${ }^{27}$ These states were formerly the capital and major cities of the Sokoto Empire, which were dismantled during British colonization.
} 
Additionally, I explore whether the effect of Sharia Law is concentrated on changes in moderate or severe levels of stunting, being underweight and wasting. Table 3.5 presents the results, with fixed effects. I find that the effect on children's nutritional outcomes is mainly concentrated on increases in severe stunting and being severely underweight. The difference-in-differences estimates show that children in states with high implementation face a substantially higher probability of being severely stunted and severely underweight (columns 2 and 4). In contrast, the results show no effect on changes in moderate stunting or being moderately underweight. The difference-in-differences estimates in the moderate stunting specification (Column 1) and moderate underweight specification (Column 3) is small and statistically insignificant. Likewise, there is no effect on moderate or severe wasting; the difference-in-differences estimates in both specifications (Column 5 and 6) are very small in magnitude and not significantly different from zero.

The results thus far indicate that Sharia Law significantly increased stunting among the treatment group. In states with high implementation, children's health outcomes worsened substantially; the impact on stunting was larger and there was an increase in the probability of being underweight.

\subsection{Empowerment}

The objective in this section is to examine the relationship between women's empowerment and Sharia Law. Women's empowerment is important for improving child growth and survival rates. Ramalingaswami et al. (1996) first highlighted that women's status is a major contributor to the problem of childhood undernutrition. As primary caregivers, women can influence the nutritional outcomes of their children through their 
own nutritional status, prenatal care usage, feeding practices and treatment of illnesses (Smith et al. 2003). However, the extent to which mothers can exert influence is dependent on empowerment. Women with higher empowerment have more control of resources, greater relative decision-making power, more access to information and health services and higher mobility (Ghulam et al. 2006, Luz and Agadjanian 2015). These factors are closely related to the mother's nutritional status, and in turn, the quality of care her children receive.

In the economic development literature, no clear definition or measurement exists for women's empowerment. Kabeer (1999) conceptualizes empowerment as the expansion of a person's ability to make strategic life choices in a setting where this ability was previously denied. The ability to make choices is measured through three dimensions: resources, agency and achievements. Keller and Mbwewe (1991) describe women's empowerment through the ability of the women to increase their own self-reliance, to make choices independently and to control resources. Duflo (2012) views women's empowerment as "improving the ability of women to access the constituents of development - in particular health, education, earning opportunities, rights and political participation" (p. 1053). Kishor (2000) defines empowerment as the amount of control women have over key aspects of their lives, measured by their resources, decision-making skills and self-reliance. Malhotra and Schuler (2005) critically examine the approaches that have been developed to measure empowerment and find that domestic decision-making, access to or control over resources and mobility of freedom are the most frequently used indicators. Based on their findings and the availability of data, I define empowerment as the ability of women to make 
household and medical decisions, have control over resources, freedom of movement and self-worth.

Defining women's empowerment is also difficult due to a lack of consensus on the terminology. In some studies, there is a distinction between women's empowerment and female autonomy (Dixon-Mueller 1998, Govindasamy and Malhotra 1996). In other studies, empowerment has often been used interchangeably with autonomy, status and agency (Jejeebhoy 2002, Kishor 2005, Basu and Koolwal 2005, Upadhyay and Hindin 2005, Lee-Rife and Edmeades 2011). Consequently, I include studies referring to women's autonomy, status and agency in reviewing the empirical literature on the relationship between empowerment and health.

\subsubsection{Empowerment and Health Outcomes of Women and Children}

Several studies have identified the links between women's empowerment and their own nutritional outcomes. For example, using data from Zimbabwe, Becker (1997) explores the effect of women's empowerment on the use of contraception and prenatal health care. Empowerment is measured by whether the woman has a role in decision-making in the purchase of household items, employment outside the home and the number of children to have. There are two stages in the regression analysis; in the first stage, the determinants of outcomes are examined and in the second stage, measures of empowerment are added to the regression analysis. The results show that empowerment has no effect on contraceptive use, but significantly increases the likelihood of prenatal care usage. Similarly, in a study in Zimbabwe, Hindin (2000) explores the relationship between women's power and nutritional outcomes by using anthropometry as a measure. Women's 
power is defined by whether the wife has a say in major household purchases, employment and the number of children to have. The results of the study suggest that women with decreased household decision-making power have a higher likelihood of lower body mass index and chronic energy deficiency.

Several studies have also pointed out the association between women's empowerment and the outcomes of children. Shroff et al. (2009) investigate the effect of maternal autonomy on child stunting in the southern state of Andra Prakesh, India. Four domains of maternal autonomy are used in the analysis, including the freedom to use discretionary money, freedom to go to the market, decision-making in different activities and attitudes to domestic violence. After controlling for socio-demographic explanatory variables, the results show that the financial autonomy measure and physical autonomy measure are significantly associated with stunting; mothers with more freedom to use finances face lower odds of stunted children, and mothers who have permission to go to the market are less likely to have stunted children. Likewise, in Nepal, Dancer and Mammohan (2009) find that women's autonomy, specifically the maternal ability to have the final say in daily household purchases, is positively associated with reduced levels of stunting among children younger than five. Further analysis of whether child nutritional outcomes differ by gender shows that the effect is particularly pronounced for boys.

Shroff et al. (2011) focus their analysis on infant outcomes by examining the impact of multiple dimensions of maternal autonomy on breastfeeding behavior and weight for length, weight for age and length for age indices in Andra Prakesh, India. Out of all the autonomy dimensions, mothers with higher levels of financial autonomy are more likely to breastfeed. In addition, the results show a positive association between household decision- 
making and infant growth. Similarly, Adhikari and Sawangdee (2011) explore the influence of maternal autonomy on infant outcomes, albeit only using infant mortality as a measure. In comparison to mothers not involved in decision-making regarding health care, the results show that the infants of mothers who are involved have a lower chance of dying. Maitra (2004) differs by examining both the effect of women's power in the household on health care usage (prenatal care, hospital delivery) and the relationship between health care usage and children's mortality outcomes in India. The author argues that jointly estimating the decision to use prenatal care, the decision to deliver in the hospital and child mortality allows for the issue of self-selection in the use of health inputs to be addressed. The paper finds that women's control over household resources and, in particular, the ability to keep money aside, has a significant effect on decisions regarding prenatal care and hospital delivery. The estimation results show that both prenatal care and hospital delivery reduce the hazard of child mortality.

\subsubsection{Data}

To examine how Sharia Law impacts the empowerment of mothers, I use data obtained from the Women's Questionnaire in the 2003 NDHS. The 1990 NDHS does not provide any data on the characteristics of respondents relating to women's status and women's participation in decision-making. The lack of data makes it impossible to implement a difference-in-differences estimation strategy.

To measure empowerment, I use questions that have been previously employed in the literature to capture woman's household and medical decision-making autonomy, control over resources (financial autonomy), physical mobility and self-worth (Hameed et al. 2014, 
Adhikari and Sawangdee 2011, Desai and Johnson 2011, Fantahun et al. 2007, Pervin et al. 2014, Shroff et al. 2009).

Household decision-making agency is measured by whether the woman makes decisions regarding large household purchases, food to be cooked daily and daily household purchases. Medical decision-making autonomy is determined through the ability of the woman to make decisions about her health care and her children's health care. Physical mobility is evaluated by whether the woman has freedom to visit family and friends and whether the woman has a problem getting permission to go for medical help (for example, visiting the health facility). The amount of control over resources is assessed through whether the woman can make decisions regarding the money her husband earns. As a proxy for self-worth, women's attitude towards wife-beating is used. Women are asked if beatings are justifiable under five different scenarios: going out, neglecting children, arguing with husband, refusing sexual relations with husband and burning the food.

For each of the questions on household and medical decision-making autonomy, I create a binary response for each indicator, where 0 represents a low level of autonomy (the woman has no role in decision-making) and 1 represents a high level of autonomy (for example, the woman solely is responsible for making decisions about her own healthcare and large household purchases). Similarly, for the questions related to physical mobility, I create indicators for each variable, where a value of 1 is assigned if the woman can freely visit family and friends, and does not have a problem obtaining permission to go for medical help. Otherwise, a value of 0 is given, to indicate that the woman's movement is restricted. For the question concerning control over resources, a binary variable is created, 
where a value of 1 represents a relatively high level of control, indicated by the woman deciding alone or jointly with her husband what to do with his earnings. Otherwise, a value of 0 is assigned to denote no say in financial resources. Regarding the questions on attitudes towards wife-beating, five binary variables are constructed, and a value of 1 is assigned to each scenario if the woman believes the beating is not justifiable, and 0 otherwise.

\subsubsection{Empirical Strategy}

To investigate the effect of Sharia Law on the women's empowerment, I estimate the following equation:

$Y_{i, s}=\delta_{1}+\delta_{2} \operatorname{Sharia}_{i, s}+\delta_{3} Z_{i, s}+\varepsilon_{i, c}$

where the dependent variable $Y_{i, s}$ represents each of the 13 indicator variables used to assess the empowerment of woman $i$ in state $s$. The focus of interest is $\delta_{2}$, the impact of Sharia Law on each empowerment outcome. The vector $Z_{i, s}$ contains controls that cover the level of household wealth, location (urban versus rural), number of members in the household, the educational level of the parents and the age of the mother. I estimate Equation (3.2) using OLS and results are reported in Tables 3.6, 3.7 and 3.8. Standard errors are presented in parentheses and the F-statistic, R-Squared value and number of observations are located in the last three rows of each table.

\subsubsection{Results}

Table 3.6 reports the results characterizing the effect of Sharia Law on household decisionmaking and control over resources. The results show that there is lower participation in decision-making on large household purchases, daily household purchases and food to be 
cooked daily among women who live in states in which Sharia Law was adopted, relative to women living in states that did not implement the reform. Additionally, the results reveal that women living in Sharia states are less likely to have control over money; the coefficient on Sharia Law in the specification in column 5 is negative and statistically significant at the 1 percent level. In all the specifications presented in columns 1 through 5 , the coefficient on age is positive and statistically significant. The position association with age suggests that the older women are, the more control they have in the household.

Table 3.7 reports the results for indicators on women's medical decision-making. Residing in a state in which the reform is present is negatively associated with women's medical-decision making ability. The results show that women in reform states are less likely to be able to make health care decisions for themselves and for their children. Regarding the physical mobility of women, the findings are inconclusive; the coefficients on the two indicators (permission to go to a health facility and visits to family and friends) are not significantly different from zero.

Table 3.8 presents the results relating to the measurement of self-worth. Women living in states in which Sharia Law is implemented have significant negative coefficients for the following scenarios: going out, refusing sex with husband and burning food. The coefficients on neglecting children and arguing with husband are not significant but retain a negative correlation. The results indicate that women living in Sharia states have a lower sense of self-worth and self-valuation, as their attitude towards beatings is reported to be that they are admissible under these scenarios.

The regression results are open to potential concerns about omitted variables and data quality. Causality cannot be assumed. However, the results do suggest a strong negative 
correlation between Sharia Law and women's empowerment with respect to household decision-making, medical decision-making and self-worth. The negative association may explain why children living in states with Sharia Law have poorer nutritional outcomes.

\subsection{Conclusion}

In this paper, I examine the effect of Sharia Law on children's nutritional outcomes in Nigeria. I exploit the variation in exposure to Sharia Law across time and state of residence to estimate the impact on child health. The difference-in-differences estimator show that the adoption of Sharia Law increased the probability of stunting in children by 8.80 percentage points. Further analysis shows that the effect is concentrated through increases in severe stunting. In states with high enforcement of Sharia Law, the likelihood of stunting increased by 20.2 percentage points and the likelihood of being underweight increased by 11.1 percentage points amongst children exposed to Sharia Law, relative to all other children.

In this paper, I also extend the analysis to explore the effect of Sharia Law on women's empowerment. I find a negative association between the implementation of Sharia Law and the empowerment of mothers with respect to household decision-making, financial autonomy and medical decision-making. While causality cannot be assumed, the negative correlation may be a potential pathway through which Sharia Law affects child health.

Ultimately, this paper provides evidence of a link between religion and child health. The findings are quite important for policy as they identify a scarcely documented factor of children's nutritional status. Child malnutrition is the largest contributor to child mortality and morbidity and is associated with cognitive impairment, less schooling, 
reduced earnings and lower economic productivity. For policymakers designing programs to reduce malnutrition, these results highlight the need to incorporate strategies that consider religious differences among children in the regions. 
Table 3.1: Summary Statistics

\begin{tabular}{lll}
\hline & $(1)$ & $(2)$ \\
& 1990 & 2003 \\
& Mean & Mean \\
\hline Poorest Q & 0.185 & 0.207 \\
Poorer Q & 0.175 & 0.208 \\
Richer Q & 0.189 & 0.206 \\
Richest Q & 0.344 & 0.183 \\
Urban & 0.470 & 0.390 \\
Household members & 7.624 & 7.446 \\
Father-Edu & 0.474 & 0.564 \\
Mother-Edu & 0.368 & 0.424 \\
Age at Birth & 26.777 & 26.737 \\
Birth Order & 3.835 & 4.038 \\
Birth Interval & 0.066 & 0.071 \\
Female & 0.502 & 0.500 \\
Stunting & 0.483 & 0.476 \\
Moderate Stunting & 0.222 & 0.202 \\
Severe Stunting & 0.259 & 0.273 \\
Underweight & 0.317 & 0.290 \\
Moderate Underweight & 0.196 & 0.184 \\
Severe Underweight & 0.120 & 0.105 \\
Wasting & 0.107 & 0.124 \\
Moderate Wasting & 0.064 & 0.077 \\
Severe Wasting & 0.043 & 0.046 \\
Sharia & 0.524 & 0.708 \\
After & 0.000 & 1.000 \\
AfterXSharia & 0.000 & 0.708 \\
High & 0.178 & 0.128 \\
AfterXHigh & 0.000 & 0.128 \\
\hline Observations & 3478 & 2949 \\
\hline
\end{tabular}


Table 3.2: Effect of Sharia Law on Children's Nutritional Outcomes

\begin{tabular}{|c|c|c|c|c|c|c|}
\hline & $\begin{array}{c}\text { (1) } \\
\text { Stunting }\end{array}$ & $\begin{array}{c}(2) \\
\text { Underweight }\end{array}$ & $\begin{array}{c}\text { (3) } \\
\text { Wasting }\end{array}$ & $\begin{array}{c}(4) \\
\text { Stunting }\end{array}$ & $\begin{array}{c}(5) \\
\text { Underweight }\end{array}$ & $\begin{array}{c}\text { (6) } \\
\text { Wasting }\end{array}$ \\
\hline After & $\begin{array}{c}-0.0903^{* * *} \\
(0.0207)\end{array}$ & $\begin{array}{l}-0.0420^{* *} \\
(0.0193)\end{array}$ & $\begin{array}{l}0.0352^{* * * *} \\
(0.0136)\end{array}$ & $\begin{array}{c}-0.0930^{* * *} \\
(0.0234)\end{array}$ & $\begin{array}{l}-0.0228 \\
(0.0216)\end{array}$ & $\begin{array}{l}0.0397^{* * *} \\
(0.0154)\end{array}$ \\
\hline Sharia & $\begin{array}{c}0.0166 \\
(0.0203)\end{array}$ & $\begin{array}{l}0.113^{* * *} \\
(0.0188)\end{array}$ & $\begin{array}{l}0.0733^{* * *} \\
(0.0133)\end{array}$ & & & \\
\hline AfterXSharia & $\begin{array}{l}0.0880^{* * *} \\
(0.0263)\end{array}$ & $\begin{array}{l}-0.0233 \\
(0.0244)\end{array}$ & $\begin{array}{l}-0.0444^{* *} \\
(0.0173)\end{array}$ & $\begin{array}{c}0.0932^{* * *} \\
(0.0298)\end{array}$ & $\begin{array}{l}-0.0308 \\
(0.0275)\end{array}$ & $\begin{array}{l}-0.0359^{*} \\
(0.0196)\end{array}$ \\
\hline Poorest Q & $\begin{array}{l}-0.0120 \\
(0.0214)\end{array}$ & $\begin{array}{l}0.0419^{* *} \\
(0.0199)\end{array}$ & $\begin{array}{l}0.0273^{*} \\
(0.0141)\end{array}$ & $\begin{array}{l}-0.0291 \\
(0.0223)\end{array}$ & $\begin{array}{c}0.0172 \\
(0.0206)\end{array}$ & $\begin{array}{c}0.0176 \\
(0.0147)\end{array}$ \\
\hline Poorer Q & $\begin{array}{l}-0.0160 \\
(0.0211)\end{array}$ & $\begin{array}{l}0.00391 \\
(0.0196)\end{array}$ & $\begin{array}{c}0.0251^{*} \\
(0.0139)\end{array}$ & $\begin{array}{l}-0.0329 \\
(0.0215)\end{array}$ & $\begin{array}{l}-0.0140 \\
(0.0199)\end{array}$ & $\begin{array}{c}0.0172 \\
(0.0141)\end{array}$ \\
\hline Richer Q & $\begin{array}{c}-0.0823^{* * *} \\
(0.0218)\end{array}$ & $\begin{array}{l}0.00170 \\
(0.0202)\end{array}$ & $\begin{array}{c}0.0111 \\
(0.0143)\end{array}$ & $\begin{array}{c}-0.0839^{* * *} \\
(0.0220)\end{array}$ & $\begin{array}{r}-0.00652 \\
(0.0203)\end{array}$ & $\begin{array}{l}0.00898 \\
(0.0144)\end{array}$ \\
\hline Richest Q & $\begin{array}{c}-0.141^{* * *} \\
(0.0242)\end{array}$ & $\begin{array}{c}-0.0644^{* * *} \\
(0.0225)\end{array}$ & $\begin{array}{c}-0.00406 \\
(0.0159)\end{array}$ & $\begin{array}{l}-0.141^{* * *} \\
(0.0250)\end{array}$ & $\begin{array}{c}-0.0810^{* * *} \\
(0.0231)\end{array}$ & $\begin{array}{l}-0.00821 \\
(0.0165)\end{array}$ \\
\hline Urban & $\begin{array}{l}-0.0432^{* *} \\
(0.0179)\end{array}$ & $\begin{array}{l}-0.0247 \\
(0.0166)\end{array}$ & $\begin{array}{c}0.0170 \\
(0.0118)\end{array}$ & $\begin{array}{l}-0.0307^{*} \\
(0.0186)\end{array}$ & $\begin{array}{l}-0.0292^{*} \\
(0.0172)\end{array}$ & $\begin{array}{c}0.0115 \\
(0.0123)\end{array}$ \\
\hline HH members & $\begin{array}{l}0.00394^{* *} \\
(0.00154)\end{array}$ & $\begin{array}{c}0.00132 \\
(0.00143)\end{array}$ & $\begin{array}{c}-0.000805 \\
(0.00101)\end{array}$ & $\begin{array}{c}0.00430^{* * *} \\
(0.00156)\end{array}$ & $\begin{array}{c}0.00141 \\
(0.00144)\end{array}$ & $\begin{array}{c}-0.000973 \\
(0.00103)\end{array}$ \\
\hline Father-Edu & $\begin{array}{c}-0.0598^{* * *} \\
(0.0161)\end{array}$ & $\begin{array}{c}-0.0382^{* *} \\
(0.0150)\end{array}$ & $\begin{array}{c}-0.00584 \\
(0.0106)\end{array}$ & $\begin{array}{c}-0.0538^{* * *} \\
(0.0162)\end{array}$ & $\begin{array}{c}-0.0298^{* *} \\
(0.0149)\end{array}$ & $\begin{array}{c}-0.00236 \\
(0.0106)\end{array}$ \\
\hline Mother-Edu & $\begin{array}{l}-0.0321^{*} \\
(0.0167)\end{array}$ & $\begin{array}{l}-0.0169 \\
(0.0155)\end{array}$ & $\begin{array}{l}-0.0113 \\
(0.0110)\end{array}$ & $\begin{array}{l}-0.0227 \\
(0.0169)\end{array}$ & $\begin{array}{l}-0.0107 \\
(0.0156)\end{array}$ & $\begin{array}{c}-0.00940 \\
(0.0111)\end{array}$ \\
\hline Age at Birth & $\begin{array}{c}-0.00744^{* * *} \\
(0.00139)\end{array}$ & $\begin{array}{c}-0.00586^{* * *} \\
(0.00129)\end{array}$ & $\begin{array}{c}0.00110 \\
(0.000912)\end{array}$ & $\begin{array}{c}-0.00739^{* * *} \\
(0.00140)\end{array}$ & $\begin{array}{c}-0.00622^{* * *} \\
(0.00130)\end{array}$ & $\begin{array}{c}0.000976 \\
(0.000923)\end{array}$ \\
\hline Birth Order & $\begin{array}{l}0.0100^{* * *} \\
(0.00386)\end{array}$ & $\begin{array}{l}0.00902^{* *} \\
(0.00358)\end{array}$ & $\begin{array}{c}-0.00191 \\
(0.00253)\end{array}$ & $\begin{array}{l}0.00995^{* *} \\
(0.00390)\end{array}$ & $\begin{array}{l}0.0104^{* * *} \\
(0.00360)\end{array}$ & $\begin{array}{l}-0.00108 \\
(0.00257)\end{array}$ \\
\hline Birth Interval & $\begin{array}{c}0.0221 \\
(0.0241)\end{array}$ & $\begin{array}{l}-0.00155 \\
(0.0223)\end{array}$ & $\begin{array}{c}0.0123 \\
(0.0158)\end{array}$ & $\begin{array}{c}0.0260 \\
(0.0241)\end{array}$ & $\begin{array}{l}0.00811 \\
(0.0223)\end{array}$ & $\begin{array}{c}0.0158 \\
(0.0158)\end{array}$ \\
\hline Female & $\begin{array}{c}-0.0602^{* * *} \\
(0.0120)\end{array}$ & $\begin{array}{c}-0.00910 \\
(0.0112)\end{array}$ & $\begin{array}{c}-0.0112 \\
(0.00791)\end{array}$ & $\begin{array}{c}-0.0619^{* * *} \\
(0.0120)\end{array}$ & $\begin{array}{l}-0.0103 \\
(0.0111)\end{array}$ & $\begin{array}{c}-0.0122 \\
(0.00791)\end{array}$ \\
\hline $\mathrm{FE}$ & no & no & no & yes & yes & yes \\
\hline F-Stat & 32.20 & 25.86 & 5.658 & 14.24 & 7.749 & 1.322 \\
\hline $\mathrm{R} 2$ & 0.0701 & 0.0571 & 0.0131 & 0.0302 & 0.0167 & 0.00289 \\
\hline Observations & 6427 & 6427 & 6427 & 6427 & 6427 & 6427 \\
\hline
\end{tabular}

Standard errors in parentheses. OLS.

${ }^{*} p<.1,{ }^{* *} p<.05,{ }^{* * *} p<.01$ 
Table 3.3: Effect of Sharia Law on Moderate and Severe Undernutrition

\begin{tabular}{|c|c|c|c|c|c|c|}
\hline & $\begin{array}{c}\text { (1) } \\
\text { Moderate } \\
\text { Stunting }\end{array}$ & $\begin{array}{c}\text { (2) } \\
\text { Severe } \\
\text { Stunting }\end{array}$ & $\begin{array}{c}\text { (3) } \\
\text { Moderate } \\
\text { Underweight }\end{array}$ & $\begin{array}{c}(4) \\
\text { Severe } \\
\text { Underweight }\end{array}$ & $\begin{array}{c}\text { (5) } \\
\text { Moderate } \\
\text { Wasting }\end{array}$ & $\begin{array}{c}(6) \\
\text { Severe } \\
\text { Wasting }\end{array}$ \\
\hline After & $\begin{array}{l}-0.0429^{* *} \\
(0.0176)\end{array}$ & $\begin{array}{c}-0.0469^{* *} \\
(0.0183)\end{array}$ & $\begin{array}{l}-0.0236 \\
(0.0167)\end{array}$ & $\begin{array}{l}-0.0199 \\
(0.0134)\end{array}$ & $\begin{array}{c}0.0190^{*} \\
(0.0110)\end{array}$ & $\begin{array}{c}0.0163^{*} \\
(0.00881)\end{array}$ \\
\hline Reform & $\begin{array}{c}-0.0422^{* *} \\
(0.0172)\end{array}$ & $\begin{array}{l}0.0590^{* * *} \\
(0.0179)\end{array}$ & $\begin{array}{c}0.0443^{* * *} \\
(0.0163)\end{array}$ & $\begin{array}{c}0.0666^{* * *} \\
(0.0131)\end{array}$ & $\begin{array}{c}0.0349^{* * *} \\
(0.0107)\end{array}$ & $\begin{array}{l}0.0383^{* * *} \\
(0.00861)\end{array}$ \\
\hline AfterXSharia & $\begin{array}{c}0.0352 \\
(0.0223)\end{array}$ & $\begin{array}{l}0.0515^{* *} \\
(0.0233)\end{array}$ & $\begin{array}{r}-0.00199 \\
(0.0212)\end{array}$ & $\begin{array}{l}-0.0185 \\
(0.0170)\end{array}$ & $\begin{array}{l}-0.0193 \\
(0.0139)\end{array}$ & $\begin{array}{c}-0.0255^{* *} \\
(0.0112)\end{array}$ \\
\hline Poorest Q & $\begin{array}{l}0.00129 \\
(0.0181)\end{array}$ & $\begin{array}{l}-0.0106 \\
(0.0189)\end{array}$ & $\begin{array}{c}0.0172 \\
(0.0173)\end{array}$ & $\begin{array}{c}0.0239^{*} \\
(0.0138)\end{array}$ & $\begin{array}{l}0.0237^{* *} \\
(0.0113)\end{array}$ & $\begin{array}{c}0.00276 \\
(0.00910)\end{array}$ \\
\hline Poorer Q & $\begin{array}{c}0.00861 \\
(0.0179)\end{array}$ & $\begin{array}{c}-0.0238 \\
(0.0186)\end{array}$ & $\begin{array}{l}-0.00541 \\
(0.0170)\end{array}$ & $\begin{array}{l}0.00858 \\
(0.0136)\end{array}$ & $\begin{array}{l}0.0269^{* *} \\
(0.0112)\end{array}$ & $\begin{array}{c}-0.00188 \\
(0.00897)\end{array}$ \\
\hline Richer Q & $\begin{array}{c}-0.0160 \\
(0.0185)\end{array}$ & $\begin{array}{c}-0.0645^{* * *} \\
(0.0193)\end{array}$ & $\begin{array}{c}0.0227 \\
(0.0176)\end{array}$ & $\begin{array}{l}-0.0204 \\
(0.0141)\end{array}$ & $\begin{array}{l}0.00510 \\
(0.0115)\end{array}$ & $\begin{array}{c}0.00592 \\
(0.00926)\end{array}$ \\
\hline Richest Q & $\begin{array}{c}0.0531^{* * *} \\
(0.0205)\end{array}$ & $\begin{array}{c}-0.0865^{* * *} \\
(0.0214)\end{array}$ & $\begin{array}{l}-0.0363^{*} \\
(0.0195)\end{array}$ & $\begin{array}{l}-0.0289^{*} \\
(0.0156)\end{array}$ & $\begin{array}{l}-0.00511 \\
(0.0128)\end{array}$ & $\begin{array}{l}0.000966 \\
(0.0103)\end{array}$ \\
\hline Urban & $\begin{array}{c}0.0149 \\
(0.0152)\end{array}$ & $\begin{array}{c}-0.0556^{* * *} \\
(0.0158)\end{array}$ & $\begin{array}{c}-0.00928 \\
(0.0145)\end{array}$ & $\begin{array}{l}-0.0161 \\
(0.0116)\end{array}$ & $\begin{array}{c}0.0101 \\
(0.00949)\end{array}$ & $\begin{array}{c}0.00711 \\
(0.00762)\end{array}$ \\
\hline HH members & $\begin{array}{c}0.00231^{*} \\
(0.00130)\end{array}$ & $\begin{array}{c}0.00175 \\
(0.00136)\end{array}$ & $\begin{array}{c}0.00184 \\
(0.00124)\end{array}$ & $\begin{array}{c}-0.000645 \\
(0.000994)\end{array}$ & $\begin{array}{l}-0.000244 \\
(0.000814)\end{array}$ & $\begin{array}{l}-0.000545 \\
(0.000654)\end{array}$ \\
\hline Father-Edu & $\begin{array}{c}-0.0130 \\
(0.0137)\end{array}$ & $\begin{array}{c}-0.0475^{* * *} \\
(0.0142)\end{array}$ & $\begin{array}{l}-0.0255^{*} \\
(0.0130)\end{array}$ & $\begin{array}{c}-0.0132 \\
(0.0104)\end{array}$ & $\begin{array}{c}0.00270 \\
(0.00853)\end{array}$ & $\begin{array}{c}-0.00802 \\
(0.00685)\end{array}$ \\
\hline Mother-Edu & $\begin{array}{r}-0.00320 \\
(0.0141)\end{array}$ & $\begin{array}{c}-0.0308^{* *} \\
(0.0147)\end{array}$ & $\begin{array}{r}-0.00723 \\
(0.0135)\end{array}$ & $\begin{array}{c}-0.0102 \\
(0.0108)\end{array}$ & $\begin{array}{l}-0.00231 \\
(0.00883)\end{array}$ & $\begin{array}{c}-0.0102 \\
(0.00709)\end{array}$ \\
\hline Age at Birth & $\begin{array}{c}-0.00162 \\
(0.00118)\end{array}$ & $\begin{array}{c}0.00558^{* * *} \\
(0.00123)\end{array}$ & $\begin{array}{c}-0.00421^{* * *} \\
(0.00112)\end{array}$ & $\begin{array}{c}-0.00152^{*} \\
(0.000896)\end{array}$ & $\begin{array}{c}0.00114 \\
(0.000734)\end{array}$ & $\begin{array}{c}0.0000282 \\
(0.000589)\end{array}$ \\
\hline Birth Order & $\begin{array}{c}-0.00158 \\
(0.00327)\end{array}$ & $\begin{array}{l}0.0113^{* * *} \\
(0.00340)\end{array}$ & $\begin{array}{c}0.00456 \\
(0.00311)\end{array}$ & $\begin{array}{c}0.00439^{*} \\
(0.00249)\end{array}$ & $\begin{array}{l}-0.00274 \\
(0.00204)\end{array}$ & $\begin{array}{l}0.000575 \\
(0.00164)\end{array}$ \\
\hline \multirow{2}{*}{$\begin{array}{l}\text { Birth } \\
\text { Interval }\end{array}$} & -0.0290 & $0.0506^{* *}$ & -0.0209 & 0.0212 & 0.0163 & -0.00364 \\
\hline & $(0.0204)$ & $(0.0213)$ & $(0.0194)$ & $(0.0155)$ & $(0.0127)$ & $(0.0102)$ \\
\hline Female & $\begin{array}{c}-0.0219^{* *} \\
(0.0102) \\
\end{array}$ & $\begin{array}{c}-0.0377^{* * *} \\
(0.0106) \\
\end{array}$ & $\begin{array}{r}-0.000482 \\
(0.00971) \\
\end{array}$ & $\begin{array}{c}-0.00804 \\
(0.00778) \\
\end{array}$ & $\begin{array}{l}-0.00578 \\
(0.00637) \\
\end{array}$ & $\begin{array}{l}-0.00575 \\
(0.00512)\end{array}$ \\
\hline FE & no & no & no & no & no & no \\
\hline F-Stat & 2.373 & 32.89 & 9.186 & 14.46 & 2.689 & 3.700 \\
\hline R2 & 0.00552 & 0.0714 & 0.0210 & 0.0327 & 0.00625 & 0.00858 \\
\hline
\end{tabular}


Observations $\quad 6427 \quad 6427$

Standard errors in parentheses. OLS.

${ }^{*} p<.1,{ }^{* *} p<.05,{ }^{* * *} p<.01$ 
Table 3.4: Effect of High Implementation on Children's Nutritional Outcomes

\begin{tabular}{|c|c|c|c|c|c|c|}
\hline & $\begin{array}{c}\text { (1) } \\
\text { Stunting }\end{array}$ & $\begin{array}{c}(2) \\
\text { Underweight }\end{array}$ & $\begin{array}{c}\text { (3) } \\
\text { Wasting }\end{array}$ & $\begin{array}{c}\text { (4) } \\
\text { Stunting }\end{array}$ & $\begin{array}{c}(5) \\
\text { Underweight }\end{array}$ & $\begin{array}{c}\text { (6) } \\
\text { Wasting }\end{array}$ \\
\hline After & $\begin{array}{l}0.0479^{* * *} \\
(0.0137)\end{array}$ & $\begin{array}{c}-0.0420^{* * *} \\
(0.0127)\end{array}$ & $\begin{array}{l}0.0264^{* * *} \\
(0.00902)\end{array}$ & $\begin{array}{l}0.0677^{* * *} \\
(0.0160)\end{array}$ & $\begin{array}{c}-0.0588^{* * *} \\
(0.0148)\end{array}$ & $\begin{array}{l}0.0226^{* *} \\
(0.0105)\end{array}$ \\
\hline High & $\begin{array}{l}-0.0242 \\
(0.0228)\end{array}$ & $\begin{array}{c}0.0344 \\
(0.0212)\end{array}$ & $\begin{array}{l}0.0369^{* *} \\
(0.0150)\end{array}$ & & & \\
\hline AfterXHigh & $\begin{array}{l}0.167^{* * *} \\
(0.0346)\end{array}$ & $\begin{array}{c}0.0518 \\
(0.0322)\end{array}$ & $\begin{array}{l}-0.0529^{* *} \\
(0.0228)\end{array}$ & $\begin{array}{l}0.202^{* * *} \\
(0.0394)\end{array}$ & $\begin{array}{l}0.111^{* * *} \\
(0.0364)\end{array}$ & $\begin{array}{l}-0.0298 \\
(0.0259)\end{array}$ \\
\hline Poorest Q & $\begin{array}{l}-0.00667 \\
(0.0215)\end{array}$ & $\begin{array}{l}0.0400^{* *} \\
(0.0200)\end{array}$ & $\begin{array}{c}0.0228 \\
(0.0142)\end{array}$ & $\begin{array}{l}-0.0244 \\
(0.0223)\end{array}$ & $\begin{array}{c}0.0230 \\
(0.0206)\end{array}$ & $\begin{array}{c}0.0178 \\
(0.0147)\end{array}$ \\
\hline Poorer Q & $\begin{array}{l}-0.0118 \\
(0.0211)\end{array}$ & $\begin{array}{l}0.00404 \\
(0.0197)\end{array}$ & $\begin{array}{c}0.0228 \\
(0.0139)\end{array}$ & $\begin{array}{l}-0.0289 \\
(0.0215)\end{array}$ & $\begin{array}{l}-0.0106 \\
(0.0199)\end{array}$ & $\begin{array}{c}0.0170 \\
(0.0141)\end{array}$ \\
\hline Richer Q & $\begin{array}{c}-0.0832^{* * *} \\
(0.0218)\end{array}$ & $\begin{array}{l}0.00442 \\
(0.0203)\end{array}$ & $\begin{array}{c}0.0125 \\
(0.0143)\end{array}$ & $\begin{array}{c}-0.0862^{* * *} \\
(0.0219)\end{array}$ & $\begin{array}{l}-0.00515 \\
(0.0203)\end{array}$ & $\begin{array}{c}0.0100 \\
(0.0144)\end{array}$ \\
\hline Richest Q & $\begin{array}{l}-0.148^{* * *} \\
(0.0242)\end{array}$ & $\begin{array}{c}-0.0697^{* * *} \\
(0.0225)\end{array}$ & $\begin{array}{l}-0.00572 \\
(0.0159)\end{array}$ & $\begin{array}{l}-0.146^{* * *} \\
(0.0250)\end{array}$ & $\begin{array}{c}-0.0799^{* * *} \\
(0.0231)\end{array}$ & $\begin{array}{l}-0.00643 \\
(0.0164)\end{array}$ \\
\hline Urban & $\begin{array}{l}-0.0405^{* *} \\
(0.0176)\end{array}$ & $\begin{array}{l}-0.0381^{* *} \\
(0.0164)\end{array}$ & $\begin{array}{l}0.00602 \\
(0.0116)\end{array}$ & $\begin{array}{l}-0.0245 \\
(0.0185)\end{array}$ & $\begin{array}{l}-0.0301^{*} \\
(0.0171)\end{array}$ & $\begin{array}{l}0.00940 \\
(0.0122)\end{array}$ \\
\hline HH members & $\begin{array}{c}0.00443^{* * *} \\
(0.00154)\end{array}$ & $\begin{array}{c}0.00229 \\
(0.00143)\end{array}$ & $\begin{array}{c}-0.000337 \\
(0.00101)\end{array}$ & $\begin{array}{c}0.00428^{* * *} \\
(0.00156)\end{array}$ & $\begin{array}{c}0.00134 \\
(0.00144)\end{array}$ & $\begin{array}{c}-0.000985 \\
(0.00103)\end{array}$ \\
\hline Father-Edu & $\begin{array}{c}-0.0654^{* * *} \\
(0.0157)\end{array}$ & $\begin{array}{c}-0.0583^{* * *} \\
(0.0146)\end{array}$ & $\begin{array}{l}-0.0178^{*} \\
(0.0103)\end{array}$ & $\begin{array}{c}-0.0503^{* * *} \\
(0.0161)\end{array}$ & $\begin{array}{l}-0.0325^{* *} \\
(0.0148)\end{array}$ & $\begin{array}{c}-0.00413 \\
(0.0106)\end{array}$ \\
\hline Mother-Edu & $\begin{array}{l}-0.0412^{* *} \\
(0.0162)\end{array}$ & $\begin{array}{l}-0.0376^{* *} \\
(0.0151)\end{array}$ & $\begin{array}{l}-0.0239^{* *} \\
(0.0107)\end{array}$ & $\begin{array}{l}-0.0183 \\
(0.0169)\end{array}$ & $\begin{array}{l}-0.00859 \\
(0.0156)\end{array}$ & $\begin{array}{l}-0.0101 \\
(0.0111)\end{array}$ \\
\hline Age at Birth & $\begin{array}{c}0.00846^{* * *} \\
(0.00134)\end{array}$ & $\begin{array}{c}-0.00769^{* * *} \\
(0.00125)\end{array}$ & $\begin{array}{l}0.0000247 \\
(0.000884)\end{array}$ & $\begin{array}{c}0.00724^{* * *} \\
(0.00140)\end{array}$ & $\begin{array}{c}-0.00580^{* * *} \\
(0.00129)\end{array}$ & $\begin{array}{c}0.00104 \\
(0.000922)\end{array}$ \\
\hline Birth Order & $\begin{array}{c}0.0122^{* * *} \\
(0.00379)\end{array}$ & $\begin{array}{l}0.0117^{* * *} \\
(0.00353)\end{array}$ & $\begin{array}{c}-0.000464 \\
(0.00250)\end{array}$ & $\begin{array}{l}0.0103^{* * *} \\
(0.00388)\end{array}$ & $\begin{array}{c}0.00945^{* * *} \\
(0.00358)\end{array}$ & $\begin{array}{l}-0.00145 \\
(0.00255)\end{array}$ \\
\hline Birth Interval & $\begin{array}{c}0.0202 \\
(0.0241)\end{array}$ & $\begin{array}{l}-0.00218 \\
(0.0224)\end{array}$ & $\begin{array}{c}0.0128 \\
(0.0159)\end{array}$ & $\begin{array}{c}0.0265 \\
(0.0241)\end{array}$ & $\begin{array}{l}0.00838 \\
(0.0222)\end{array}$ & $\begin{array}{c}0.0158 \\
(0.0158)\end{array}$ \\
\hline Female & $\begin{array}{c}-0.0599^{* * *} \\
(0.0120) \\
\end{array}$ & $\begin{array}{r}-0.00897 \\
(0.0112) \\
\end{array}$ & $\begin{array}{c}-0.0113 \\
(0.00793)\end{array}$ & $\begin{array}{c}-0.0616^{* * *} \\
(0.0120)\end{array}$ & $\begin{array}{l}-0.0104 \\
(0.0111) \\
\end{array}$ & $\begin{array}{c}-0.0123 \\
(0.00791)\end{array}$ \\
\hline FE & no & no & no & yes & yes & yes \\
\hline F-Stat & 32.59 & 23.52 & 4.031 & 15.45 & 8.331 & 1.177 \\
\hline $\mathrm{R} 2$ & 0.0708 & 0.0522 & 0.00934 & 0.0327 & 0.0179 & 0.00257 \\
\hline Observations & 6427 & 6427 & 6427 & 6427 & 6427 & 6427 \\
\hline
\end{tabular}


Table 3.5: Effect of High Implementation on Moderate and Severe Undernutrition

\begin{tabular}{|c|c|c|c|c|c|c|}
\hline & (1) & (2) & (3) & (4) & (5) & (6) \\
\hline & $\begin{array}{c}\text { Moderate } \\
\text { Stunting }\end{array}$ & Severe Stunting & $\begin{array}{l}\text { Moderate } \\
\text { Underweight }\end{array}$ & $\begin{array}{c}\text { Severe } \\
\text { Underweight }\end{array}$ & $\begin{array}{c}\text { Moderate } \\
\text { Wasting }\end{array}$ & $\begin{array}{c}\text { Severe } \\
\text { Wasting }\end{array}$ \\
\hline After & $\begin{array}{c}-0.0251^{*} \\
(0.0136)\end{array}$ & $\begin{array}{c}-0.0438^{* * *} \\
(0.0141)\end{array}$ & $\begin{array}{l}-0.0209 \\
(0.0129)\end{array}$ & $\begin{array}{c}-0.0394^{* * *} \\
(0.0103)\end{array}$ & $\begin{array}{c}0.0168^{* *} \\
(0.00850)\end{array}$ & $\begin{array}{c}0.00560 \\
(0.00683)\end{array}$ \\
\hline AfterXHigh & $\begin{array}{c}-0.0396 \\
(0.0335)\end{array}$ & $\begin{array}{l}0.240^{* * *} \\
(0.0347)\end{array}$ & $\begin{array}{c}0.0458 \\
(0.0318)\end{array}$ & $\begin{array}{c}0.0715^{* * *} \\
(0.0254)\end{array}$ & $\begin{array}{l}-0.0236 \\
(0.0209)\end{array}$ & $\begin{array}{r}-0.00620 \\
(0.0168)\end{array}$ \\
\hline Poorest Q & $\begin{array}{c}0.00308 \\
(0.0190)\end{array}$ & $\begin{array}{c}-0.0242 \\
(0.0196)\end{array}$ & $\begin{array}{l}0.00509 \\
(0.0180)\end{array}$ & $\begin{array}{c}0.0181 \\
(0.0144)\end{array}$ & $\begin{array}{c}0.0154 \\
(0.0118)\end{array}$ & $\begin{array}{c}0.00168 \\
(0.00951)\end{array}$ \\
\hline Poorer Q & $\begin{array}{c}0.00652 \\
(0.0183)\end{array}$ & $\begin{array}{l}-0.0344^{*} \\
(0.0189)\end{array}$ & $\begin{array}{l}-0.0120 \\
(0.0173)\end{array}$ & $\begin{array}{l}0.00108 \\
(0.0139)\end{array}$ & $\begin{array}{c}0.0212^{*} \\
(0.0114)\end{array}$ & $\begin{array}{l}-0.00423 \\
(0.00916)\end{array}$ \\
\hline Richer Q & $\begin{array}{c}-0.0229 \\
(0.0186)\end{array}$ & $\begin{array}{c}-0.0616^{* * *} \\
(0.0193)\end{array}$ & $\begin{array}{c}0.0171 \\
(0.0177)\end{array}$ & $\begin{array}{l}-0.0219 \\
(0.0141)\end{array}$ & $\begin{array}{l}0.00274 \\
(0.0116)\end{array}$ & $\begin{array}{c}0.00727 \\
(0.00935)\end{array}$ \\
\hline Richest Q & $\begin{array}{c}0.0563^{* * *} \\
(0.0212)\end{array}$ & $\begin{array}{c}-0.0887^{* * *} \\
(0.0220)\end{array}$ & $\begin{array}{c}-0.0458^{* *} \\
(0.0202)\end{array}$ & $\begin{array}{c}-0.0351^{* *} \\
(0.0161)\end{array}$ & $\begin{array}{l}-0.00666 \\
(0.0132)\end{array}$ & $\begin{array}{c}0.000173 \\
(0.0106)\end{array}$ \\
\hline Urban & $\begin{array}{c}0.0281^{*} \\
(0.0158)\end{array}$ & $\begin{array}{c}-0.0495^{* * *} \\
(0.0163)\end{array}$ & $\begin{array}{l}-0.00774 \\
(0.0150)\end{array}$ & $\begin{array}{l}-0.0224^{*} \\
(0.0120)\end{array}$ & $\begin{array}{c}0.00412 \\
(0.00984)\end{array}$ & $\begin{array}{c}0.00545 \\
(0.00791)\end{array}$ \\
\hline HH members & $\begin{array}{c}0.00197 \\
(0.00133)\end{array}$ & $\begin{array}{c}0.00242^{*} \\
(0.00137)\end{array}$ & $\begin{array}{c}0.00181 \\
(0.00126)\end{array}$ & $\begin{array}{l}-0.000642 \\
(0.00101)\end{array}$ & $\begin{array}{l}-0.000359 \\
(0.000828)\end{array}$ & $\begin{array}{l}-0.000603 \\
(0.000665)\end{array}$ \\
\hline Father-Edu & $\begin{array}{r}-0.00961 \\
(0.0137)\end{array}$ & $\begin{array}{c}-0.0415^{* * *} \\
(0.0141)\end{array}$ & $\begin{array}{l}-0.0214^{*} \\
(0.0130)\end{array}$ & $\begin{array}{l}-0.0116 \\
(0.0104)\end{array}$ & $\begin{array}{c}0.00483 \\
(0.00852)\end{array}$ & $\begin{array}{l}-0.00852 \\
(0.00685)\end{array}$ \\
\hline Mother-Edu & $\begin{array}{c}0.000423 \\
(0.0143)\end{array}$ & $\begin{array}{c}-0.0206 \\
(0.0148)\end{array}$ & $\begin{array}{r}-0.00280 \\
(0.0136)\end{array}$ & $\begin{array}{r}-0.00589 \\
(0.0109)\end{array}$ & $\begin{array}{c}-0.00166 \\
(0.00895)\end{array}$ & $\begin{array}{l}-0.00966 \\
(0.00719)\end{array}$ \\
\hline Age at Birth & $\begin{array}{l}-0.00176 \\
(0.00119)\end{array}$ & $\begin{array}{c}0.00523^{* * *} \\
(0.00123)\end{array}$ & $\begin{array}{c}-0.00430^{* * *} \\
(0.00113)\end{array}$ & $\begin{array}{c}-0.00136 \\
(0.000903)\end{array}$ & $\begin{array}{c}0.00107 \\
(0.000743)\end{array}$ & $\begin{array}{c}0.00000570 \\
(0.000597)\end{array}$ \\
\hline Birth Order & $\begin{array}{l}0.000839 \\
(0.00330)\end{array}$ & $\begin{array}{l}0.0108^{* * *} \\
(0.00341)\end{array}$ & $\begin{array}{c}0.00514 \\
(0.00313)\end{array}$ & $\begin{array}{c}0.00426^{*} \\
(0.00250)\end{array}$ & $\begin{array}{l}-0.00220 \\
(0.00206)\end{array}$ & $\begin{array}{l}0.000477 \\
(0.00165)\end{array}$ \\
\hline Birth Interval & $\begin{array}{c}-0.0296 \\
(0.0205)\end{array}$ & $\begin{array}{c}0.0555^{* * *} \\
(0.0212)\end{array}$ & $\begin{array}{l}-0.0158 \\
(0.0194)\end{array}$ & $\begin{array}{c}0.0259^{*} \\
(0.0155)\end{array}$ & $\begin{array}{c}0.0180 \\
(0.0128)\end{array}$ & $\begin{array}{r}-0.00187 \\
(0.0103)\end{array}$ \\
\hline Female & $\begin{array}{c}-0.0226^{* *} \\
(0.0102)\end{array}$ & $\begin{array}{c}-0.0383^{* * *} \\
(0.0106)\end{array}$ & $\begin{array}{l}-0.00155 \\
(0.00970)\end{array}$ & $\begin{array}{c}-0.00824 \\
(0.00775)\end{array}$ & $\begin{array}{c}-0.00640 \\
(0.00638)\end{array}$ & $\begin{array}{l}-0.00618 \\
(0.00512)\end{array}$ \\
\hline FE & yes & yes & yes & yes & yes & yes \\
\hline F-Stat & 2.393 & 13.88 & 3.707 & 5.169 & 1.168 & 0.765 \\
\hline R2 & 0.00521 & 0.0295 & 0.00806 & 0.0112 & 0.00255 & 0.00167 \\
\hline Observations & 6427 & 6427 & 6427 & 6427 & 6427 & 6427 \\
\hline
\end{tabular}

Standard errors in parentheses. OLS.

${ }^{*} p<.1,{ }^{* *} p<.05,{ }^{* * *} p<.01$ 
Table 3.6: Effect of Sharia Law on Household Decision-making

\begin{tabular}{|c|c|c|c|c|c|}
\hline & $\begin{array}{c}\text { (1) } \\
\text { HH purchases }\end{array}$ & $\begin{array}{c}(2) \\
\text { Visits }\end{array}$ & $\begin{array}{c}\text { (3) } \\
\text { Food to be cooked }\end{array}$ & $\begin{array}{c}\text { (4) } \\
\text { Daily HH purchases }\end{array}$ & $\begin{array}{c}\text { (5) } \\
\text { Spends money }\end{array}$ \\
\hline Sharia & $\begin{array}{l}-0.0589^{* * *} \\
(0.0123)\end{array}$ & $\begin{array}{l}-0.0174 \\
(0.0217)\end{array}$ & $\begin{array}{l}-0.122^{* * *} \\
(0.0243)\end{array}$ & $\begin{array}{l}-0.222^{* * *} \\
(0.0176)\end{array}$ & $\begin{array}{c}-0.0993^{* * *} \\
(0.0263)\end{array}$ \\
\hline Poorest Q & $\begin{array}{l}-0.0184 \\
(0.0160)\end{array}$ & $\begin{array}{c}0.0254 \\
(0.0281)\end{array}$ & $\begin{array}{l}-0.0241 \\
(0.0315)\end{array}$ & $\begin{array}{l}-0.0517^{* *} \\
(0.0228)\end{array}$ & $\begin{array}{l}-0.131^{* * *} \\
(0.0341)\end{array}$ \\
\hline Poorer Q & $\begin{array}{l}-0.0214 \\
(0.0157)\end{array}$ & $\begin{array}{l}-0.0185 \\
(0.0277)\end{array}$ & $\begin{array}{l}-0.0380 \\
(0.0310)\end{array}$ & $\begin{array}{l}-0.0190 \\
(0.0225)\end{array}$ & $\begin{array}{l}-0.0464 \\
(0.0337)\end{array}$ \\
\hline Richer Q & $\begin{array}{l}-0.0162 \\
(0.0164)\end{array}$ & $\begin{array}{c}0.0754^{* * *} \\
(0.0289)\end{array}$ & $\begin{array}{l}-0.0134 \\
(0.0323)\end{array}$ & $\begin{array}{l}-0.0144 \\
(0.0235)\end{array}$ & $\begin{array}{l}-0.0338 \\
(0.0351)\end{array}$ \\
\hline Richest Q & $\begin{array}{l}-0.0449^{* *} \\
(0.0192)\end{array}$ & $\begin{array}{l}-0.0531 \\
(0.0338)\end{array}$ & $\begin{array}{c}0.0229 \\
(0.0378)\end{array}$ & $\begin{array}{c}0.0247 \\
(0.0275)\end{array}$ & $\begin{array}{c}0.0317 \\
(0.0411)\end{array}$ \\
\hline Urban & $\begin{array}{l}-0.00868 \\
(0.0131)\end{array}$ & $\begin{array}{l}0.00304 \\
(0.0231)\end{array}$ & $\begin{array}{c}0.0497^{*} \\
(0.0258)\end{array}$ & $\begin{array}{l}-0.0298 \\
(0.0188)\end{array}$ & $\begin{array}{l}-0.0253 \\
(0.0280)\end{array}$ \\
\hline HH members & $\begin{array}{c}0.00100 \\
(0.00133)\end{array}$ & $\begin{array}{l}0.000363 \\
(0.00234)\end{array}$ & $\begin{array}{c}-0.00575^{* *} \\
(0.00262)\end{array}$ & $\begin{array}{l}-0.00158 \\
(0.00191)\end{array}$ & $\begin{array}{l}0.00673^{* *} \\
(0.00285)\end{array}$ \\
\hline Father-Edu & $\begin{array}{c}0.000321 \\
(0.0123)\end{array}$ & $\begin{array}{l}0.00691 \\
(0.0216)\end{array}$ & $\begin{array}{l}0.0597^{* *} \\
(0.0242)\end{array}$ & $\begin{array}{r}-0.00212 \\
(0.0176)\end{array}$ & $\begin{array}{l}-0.0106 \\
(0.0263)\end{array}$ \\
\hline Mother-Edu & $\begin{array}{l}0.0413^{* * *} \\
(0.0129)\end{array}$ & $\begin{array}{l}0.0506^{* *} \\
(0.0227)\end{array}$ & $\begin{array}{c}0.0403 \\
(0.0255)\end{array}$ & $\begin{array}{c}0.0650^{* * *} \\
(0.0185)\end{array}$ & $\begin{array}{c}0.0186 \\
(0.0276)\end{array}$ \\
\hline Age & $\begin{array}{l}0.00198^{* * *} \\
(0.000732)\end{array}$ & $\begin{array}{l}0.00321^{* *} \\
(0.00129)\end{array}$ & $\begin{array}{l}0.00358^{* *} \\
(0.00144)\end{array}$ & $\begin{array}{c}0.00420^{* * *} \\
(0.00105)\end{array}$ & $\begin{array}{c}0.00704^{* * *} \\
(0.00157)\end{array}$ \\
\hline $\mathrm{FE}$ & no & no & no & No & no \\
\hline F-Stat & 6.606 & 2.567 & 13.54 & 33.77 & 8.927 \\
\hline $\mathrm{R} 2$ & 0.0300 & 0.0119 & 0.0597 & 0.137 & 0.0402 \\
\hline Observations & 2145 & 2145 & 2145 & 2145 & 2145 \\
\hline
\end{tabular}

Standard errors in parentheses. OLS.

${ }^{*} p<.1,{ }^{* *} p<.05,{ }^{* * *} p<.01$ 
Table 3.7: Effect of Sharia Law on Medical Decision-making

\begin{tabular}{|c|c|c|c|}
\hline & $\begin{array}{c}(1) \\
\text { Own Healthcare }\end{array}$ & $\begin{array}{c}(2) \\
\text { Medical care for child }\end{array}$ & $\begin{array}{l}\text { (3) } \\
\text { Permission to go } \\
\text { for medical help }\end{array}$ \\
\hline Sharia & $\begin{array}{l}-0.189^{* * *} \\
(0.0170)\end{array}$ & $\begin{array}{l}-0.154^{* * *} \\
(0.0236)\end{array}$ & $\begin{array}{l}-0.00391 \\
(0.0213)\end{array}$ \\
\hline Poorest Q & $\begin{array}{l}-0.00983 \\
(0.0220)\end{array}$ & $\begin{array}{l}-0.115^{* * *} \\
(0.0306)\end{array}$ & $\begin{array}{l}-0.100^{* * *} \\
(0.0275)\end{array}$ \\
\hline Poorer Q & $\begin{array}{l}-0.0383^{*} \\
(0.0217)\end{array}$ & $\begin{array}{c}-0.0821^{* * *} \\
(0.0302)\end{array}$ & $\begin{array}{l}-0.0654^{* *} \\
(0.0272)\end{array}$ \\
\hline Richer Q & $\begin{array}{c}0.0261 \\
(0.0226)\end{array}$ & $\begin{array}{l}0.0838^{* * *} \\
(0.0315)\end{array}$ & $\begin{array}{l}0.0599^{* *} \\
(0.0283)\end{array}$ \\
\hline Richest Q & $\begin{array}{l}-0.0106 \\
(0.0264)\end{array}$ & $\begin{array}{l}0.176^{* * *} \\
(0.0369)\end{array}$ & $\begin{array}{l}0.0901^{* * *} \\
(0.0331)\end{array}$ \\
\hline Urban & $\begin{array}{l}-0.00452 \\
(0.0181)\end{array}$ & $\begin{array}{c}0.0409 \\
(0.0252)\end{array}$ & $\begin{array}{c}0.0182 \\
(0.0226)\end{array}$ \\
\hline HH members & $\begin{array}{l}-0.000606 \\
(0.00183)\end{array}$ & $\begin{array}{l}0.00444^{*} \\
(0.00256)\end{array}$ & $\begin{array}{c}0.00201 \\
(0.00230)\end{array}$ \\
\hline Father-Edu & $\begin{array}{l}0.00628 \\
(0.0169)\end{array}$ & $\begin{array}{l}0.0983^{* * *} \\
(0.0236)\end{array}$ & $\begin{array}{l}0.0544^{* *} \\
(0.0212)\end{array}$ \\
\hline Mother-Edu & $\begin{array}{c}0.0174 \\
(0.0178)\end{array}$ & $\begin{array}{l}0.103^{* * *} \\
(0.0248)\end{array}$ & $\begin{array}{l}0.0995^{* * *} \\
(0.0223)\end{array}$ \\
\hline Age & $\begin{array}{l}0.00494^{* * *} \\
(0.00101)\end{array}$ & $\begin{array}{l}0.00640^{* * *} \\
(0.00141)\end{array}$ & $\begin{array}{l}0.00521^{* * *} \\
(0.00126)\end{array}$ \\
\hline $\begin{array}{l}\text { FE } \\
\text { F-Stat } \\
\text { R2 } \\
\text { Observations }\end{array}$ & $\begin{array}{c}\text { no } \\
23.71 \\
0.100 \\
2145\end{array}$ & $\begin{array}{c}\text { No } \\
55.88 \\
0.208 \\
2145\end{array}$ & $\begin{array}{c}\text { no } \\
23.75 \\
0.100 \\
2145\end{array}$ \\
\hline
\end{tabular}

Standard errors in parentheses. OLS.

${ }^{*} p<.1,{ }^{* *} p<.05,{ }^{* * *} p<.01$ 
Table 3.8: Effect of Sharia Law on Attitudes towards Violence

\begin{tabular}{|c|c|c|c|c|c|}
\hline & $\begin{array}{c}\text { (1) } \\
\text { Goes out }\end{array}$ & $\begin{array}{c}(2) \\
\text { Neglects children }\end{array}$ & $\begin{array}{c}\text { (3) } \\
\text { Argues with husband }\end{array}$ & $\begin{array}{c}\text { (4) } \\
\text { Refuses sex } \\
\text { with husband }\end{array}$ & $\begin{array}{c}\text { (5) } \\
\text { Burns food }\end{array}$ \\
\hline Sharia & $\begin{array}{l}-0.275^{* * *} \\
(0.0238)\end{array}$ & $\begin{array}{l}-0.0361 \\
(0.0260)\end{array}$ & $\begin{array}{l}-0.000748 \\
(0.0261)\end{array}$ & $\begin{array}{l}-0.198^{* * *} \\
(0.0252)\end{array}$ & $\begin{array}{l}-0.0555^{* *} \\
(0.0249)\end{array}$ \\
\hline Poorest Q & $\begin{array}{c}-0.0906^{* * *} \\
(0.0308)\end{array}$ & $\begin{array}{l}-0.199^{* * *} \\
(0.0337)\end{array}$ & $\begin{array}{l}-0.225^{* * *} \\
(0.0338)\end{array}$ & $\begin{array}{l}-0.155^{* * *} \\
(0.0327)\end{array}$ & $\begin{array}{l}-0.217^{* * *} \\
(0.0322)\end{array}$ \\
\hline Poorer Q & $\begin{array}{l}-0.0298 \\
(0.0304)\end{array}$ & $\begin{array}{c}-0.0972^{* * *} \\
(0.0333)\end{array}$ & $\begin{array}{c}-0.0965^{* * *} \\
(0.0334)\end{array}$ & $\begin{array}{l}-0.0709^{* *} \\
(0.0322)\end{array}$ & $\begin{array}{c}-0.0837^{* * *} \\
(0.0318)\end{array}$ \\
\hline Richer Q & $\begin{array}{c}0.0214 \\
(0.0317)\end{array}$ & $\begin{array}{l}0.0634^{*} \\
(0.0347)\end{array}$ & $\begin{array}{l}0.0684^{* *} \\
(0.0348)\end{array}$ & $\begin{array}{l}0.0791^{* *} \\
(0.0336)\end{array}$ & $\begin{array}{l}0.129^{* * *} \\
(0.0331)\end{array}$ \\
\hline Richest Q & $\begin{array}{l}0.191^{* * *} \\
(0.0371)\end{array}$ & $\begin{array}{l}0.151^{* * *} \\
(0.0406)\end{array}$ & $\begin{array}{l}0.182^{* * *} \\
(0.0407)\end{array}$ & $\begin{array}{l}0.201^{* * *} \\
(0.0393)\end{array}$ & $\begin{array}{l}0.232^{* * *} \\
(0.0387)\end{array}$ \\
\hline Urban & $\begin{array}{c}-0.0827^{* * *} \\
(0.0253)\end{array}$ & $\begin{array}{l}-0.0673^{* *} \\
(0.0277)\end{array}$ & $\begin{array}{l}-0.108^{* * *} \\
(0.0278)\end{array}$ & $\begin{array}{c}-0.0695^{* * *} \\
(0.0268)\end{array}$ & $\begin{array}{l}-0.118^{* * *} \\
(0.0264)\end{array}$ \\
\hline HH members & $\begin{array}{l}0.00763^{* * *} \\
(0.00257)\end{array}$ & $\begin{array}{l}-0.00636^{* *} \\
(0.00281)\end{array}$ & $\begin{array}{l}-0.00451 \\
(0.00282)\end{array}$ & $\begin{array}{c}-0.00818^{* * *} \\
(0.00273)\end{array}$ & $\begin{array}{l}0.00744^{* * *} \\
(0.00269)\end{array}$ \\
\hline Father-Edu & $\begin{array}{c}0.0162 \\
(0.0237)\end{array}$ & $\begin{array}{c}0.0191 \\
(0.0260)\end{array}$ & $\begin{array}{l}-0.00233 \\
(0.0261)\end{array}$ & $\begin{array}{l}-0.00275 \\
(0.0252)\end{array}$ & $\begin{array}{l}0.00643 \\
(0.0248)\end{array}$ \\
\hline Mother-Edu & $\begin{array}{l}0.0599^{* *} \\
(0.0250)\end{array}$ & $\begin{array}{c}0.0106 \\
(0.0273)\end{array}$ & $\begin{array}{c}0.0399 \\
(0.0274)\end{array}$ & $\begin{array}{c}0.0413 \\
(0.0265)\end{array}$ & $\begin{array}{l}0.00897 \\
(0.0261)\end{array}$ \\
\hline Age & $\begin{array}{l}0.00341^{* *} \\
(0.00141)\end{array}$ & $\begin{array}{c}0.00296^{*} \\
(0.00155)\end{array}$ & $\begin{array}{l}0.00365^{* *} \\
(0.00155)\end{array}$ & $\begin{array}{l}0.00306^{* *} \\
(0.00150)\end{array}$ & $\begin{array}{l}0.00324^{* *} \\
(0.00148)\end{array}$ \\
\hline FE & no & no & No & no & no \\
\hline F-Stat & 40.34 & 13.70 & 14.64 & 29.81 & 21.61 \\
\hline $\mathrm{R} 2$ & 0.159 & 0.0603 & 0.0642 & 0.123 & 0.0919 \\
\hline Observations & 2145 & 2145 & 2145 & 2145 & 2145 \\
\hline
\end{tabular}

Standard errors in parentheses. OLS.

${ }^{*} p<.1,{ }^{* *} p<.05,{ }^{* * *} p<.01$ 


\section{REFERENCES}

Adejumo, A. O., \& Fwacn, R. P. (2011). The dynamics of sharia adoption in northern Nigeria and women rights: An ethical analysis. Global Journal of African Studies, 1(1), 24-29.

Adhikari, R., \& Sawangdee, Y. (2011). Influence of women's autonomy on infant mortality in Nepal. Reproductive Health, 8(1), 7.

Ahmed, S. A., Penhale, W. J., \& Talal, N. (1985). Sex hormones, immune responses, and autoimmune diseases. Mechanisms of sex hormone action. The American Journal of Pathology, 121(3), 531.

Ahmed, T., \& Islam, S. (1984). Impact of Socioeconomic Condition and Maternal Education on Nutritional Status of Children. Dhaka Shishu Hospital J., September, 16.

Ahmed, S., Khan, S., Alia, M., \& Noushad, S. (2013). PSYCHOLOGICAL IMPACT EVALUATION OF EARLY MARRIAGES. International Journal of Endorsing Health Science Research, 1(2),84-86

Alkema, L., Chou, D., Hogan, D., Zhang, S., Moller, A. B., Gemmill, A., ... \& Say, L. (2016). Global, regional, and national levels and trends in maternal mortality between 1990 and 2015, with scenario-based projections to 2030: a systematic analysis by the UN Maternal Mortality Estimation Inter-Agency Group. The Lancet, 387(10017), 462-474.

Arnold, F., Choe, M. K., \& Roy, T. K. (1998). Son preference, the family-building process and child mortality in India. Population Studies, 52(3), 301-315.

Asada, H., Matsumoto, J., \& Rahman, R. (2005). Impact of recent severe floods on rice production in Bangladesh. Geographical Review of Japan, 78(12), 783-793.

Asada, H., \& Matsumoto, J. (2009). Effects of rainfall variation on rice production in the Ganges-Brahmaputra Basin. Climate Research, 38(3), 249-260.

Asaduzzaman, M., Ringler, C., Thurlow, J., \& Alam, S. (2010, May 26). Investing in Crop Agriculture in Bangladesh for Higher Growth and Productivity, and Adaptation to Climate Change. Retrieved February 2, 2016, from https://bangladeshfoodsecurity.files.wordpress.com/2010/05/investing.pdf

Atkin, D. (2009). Working for the future: Female factory work and child health in Mexico. Unpublished Manuscript, Yale University.

Bairagi, R., \& Chowdhury, M. K. (1994). Socioeconomic and anthropometric status, and mortality of young children in rural Bangladesh. International Journal of Epidemiology, 23(6), 1179-1184. 
Bank, B. (2014). Annual Report 2013-14 Dhaka, Bangladesh. Retrieved February 2,2015, from: http:// www.bangladesh-bank.org/econdata/index.php

Bangladesh, D. H. S. (2007). National Institute of Population Research and Training (NIPORT), Mitra and Associates, and Macro International. (2009). Calverton, Maryland: Bangladesh Demographic and Health Survey.

Basu, A. M. (1992). Culture, the status of women, and demographic behaviour: illustrated with the case of India.Oxford, UK: Clarendon Press.

Basu, A. M., \& Koolwal, G. B. (2005). Two concepts of female empowerment: Some leads from DHS data on women's status and reproductive health. A focus on genderCollected papers on gender using DHS data, ORC Macro, Calverton MD, 15-33.

Baqui, A. H., Black, R. E., Arifeen, S. E., Hill, K., Mitra, S. N., \& Al Sabir, A. (1998). Causes of childhood deaths in Bangladesh: results of a nationwide verbal autopsy study. Bulletin of the World Health Organization, 76(2), 161.

Beaman, L., Duflo, E., Pande, R., \& Topalova, P. (2012). Female leadership raises aspirations and educational attainment for girls: A policy experiment in India. Science, 335(6068), 582-586.

Becher, H., Müller, O., Jahn, A., Gbangou, A., Kynast-Wolf, G., \& Kouyaté, B. (2004). Risk factors of infant and child mortality in rural Burkina Faso. Bulletin of the World Health Organization, 82(4), 265-273.

Becker, S. (1997). Incorporating women's empowerment in studies of reproductive health: an example from Zimbabwe, paper presented at the Seminar on Female Empowerment \& Demographic Processes. University of Lund.

Beegle, K., Frankenberg, E., \& Thomas, D. (2001). Bargaining power within couples and use of prenatal and delivery care in Indonesia. Studies in Family Planning, 32(2), 130146.

Behrman, J. R., Foster, A. D., Rosenweig, M. R., \& Vashishtha, P. (1999). Women's schooling, home teaching, and economic growth. Journal of Political Economy, 107(4), 682-714.

Behrman, J. R., \& Rosenzweig, M. R. (2005). Does increasing women's schooling raise the schooling of the next generation? Reply. The American economic review, 95(5), 1745-1751.

Bhalotra, S., Valente, C., \& Van Soest, A. (2010). The puzzle of Muslim advantage in child survival in India. Journal of Health Economics, 29(2), 191-204. 
Black, M. (2001). Early Marriage: Child Spouses. Innocenti Digest. UNICEF Innocenti Research Centre.

Black, R. E., Allen, L. H., Bhutta, Z. A., Caulfield, L. E., De Onis, M., Ezzati, M., ... \& Maternal and Child Undernutrition Study Group. (2008). Maternal and child undernutrition: global and regional exposures and health consequences. The Lancet, 371(9608), 243-260.

Black, R. E., Victora, C. G., Walker, S. P., Bhutta, Z. A., Christian, P., De Onis, M., ... \& Uauy, R. (2013). Maternal and child undernutrition and overweight in low-income and middle-income countries. The Lancet, 382(9890), 427-451.

Blair, G., Christine Fair, C., Malhotra, N., \& Shapiro, J. N. (2013). Poverty and support for militant politics: Evidence from Pakistan. American Journal of Political Science, 57(1), 30-48.

Bloss, E., Wainaina, F., \& Bailey, R. C. (2004). Prevalence and predictors of underweight, stunting, and wasting among children aged 5 and under in western Kenya. Journal of Tropical Pediatrics, 50(5), 260-270.

Bose, K., Biswas, S., Bisai, S., Ganguli, S., Khatun, A., Mukhopadhyay, A., \& Bhadra, M. (2007). Stunting, underweight and wasting among Integrated Child Development Services (ICDS) scheme children aged 3-5 years of Chapra, Nadia District, West Bengal, India. Maternal \& Child Nutrition, 3(3), 216-221.

Brammer, H. (1990). Floods in Bangladesh: geographical background to the 1987 and 1988 floods. Geographical Journal, 12-22.

Burke, M., Gong, E., \& Jones, K. (2015). Income shocks and HIV in Africa. The Economic Journal, 125(585), 1157-1189.

Carbone-López, K., Kruttschnitt, C., \& Macmillan, R. (2006). Patterns of intimate partner violence and their associations with physical health, psychological distress, and substance use. Public Health Reports, 121(4), 382-392.

Cavallo, E., Galiani, S., Noy, I., \& Pantano, J. (2013). Catastrophic natural disasters and economic growth. Review of Economics and Statistics, 95(5), 1549-1561.

Cayan, D. R., Das, T., Pierce, D. W., Barnett, T. P., Tyree, M., \& Gershunov, A. (2010). Future dryness in the southwest US and the hydrology of the early 21 st century drought. Proceedings of the National Academy of Sciences, 107(50), 21271-21276.

Chen, M. (1983). A Quiet Revolution: Women in Transition in Rural Bangladesh. Cambridge, MA: Schenkman Publishing Company 
Chen, L. C., Huq, E., \& D'Souza, S. (1981). Sex bias in the family allocation of food and health care in rural Bangladesh. Population and Development Review, 55-70.

Child Marriage Restraint Act, 1929 (Act No. XIX of 1929). Retrieved April 21, 2015, from http://bdlaws.minlaw.gov.bd/print_sections_all.php?id=149

Chowdhury, F. D. (2004). The socio-cultural context of child marriage in a Bangladeshi village. International Journal of Social Welfare, 13(3), 244-253.

Chowdhury, H. R., Thompson, S., Ali, M., Alam, N., Yunus, M., \& Streatfield, P. K. (2010). Causes of neonatal deaths in a rural subdistrict of Bangladesh: implications for intervention. Journal of Health, Population, and Nutrition, 28(4), 375.

Cleland, J. G., \& Sathar, Z. A. (1984). The effect of birth spacing on childhood mortality in Pakistan. Population Studies, 38(3), 401-418.

Corno, L., \& Voena, A. (2015). Selling daughters: age of marriage, income shocks and bride price tradition. Rockwool Foundation Research Unit.

Corno, L., Hildebrandt, N., \& Voena, A. (2016). Weather Shocks, Age of Marriage and the Direction of Marriage Payments. Unpublished Manuscript.

Cunado, J., \& Ferreira, S. (2014). The macroeconomic impacts of natural disasters: the case of floods. Land Economics, 90(1), 149-168.

Chao, T. C. (1996). Female sex hormones and the immune system. Chang Gung Medical Journal, 19, 95-106.

D'Souza, S., \& Chen, L. C. (1980). Sex differentials in mortality in rural Bangladesh. Population and Development Review, 257-270.

Dancer, D., \& Rammohan, A. (2009). Maternal autonomy and child nutrition: evidence from rural Nepal. Indian Growth and Development Review, 2(1), 18-38.

Das Gupta, M. (1990). Death clustering, mothers' education and the determinants of child mortality in rural Punjab, India. Population Studies, 44(3), 489-505.

Desai, S., \& Johnson, K. (2005). Women's Decisionmaking and Child Health: Familial and social hierarchies. A focus on gender: Collected papers on gender using DHS data, 2005, 55-68.

Deshmukh, P. R., Sinha, N., \& Dongre, A. R. (2013). Social determinants of stunting in rural area of Wardha, Central India. Medical Journal Armed Forces India, 69(3), 213217. 
Dewey, K. G., Hawck, M. G., Brown, K. H., Lartey, A., Cohen, R. J., \& Peerson, J. M. (2005). Infant weight-for-length is positively associated with subsequent linear growth across four different populations. Maternal \& Child Nutrition, 1(1), 11-20.

Dewey, K. G., \& Begum, K. (2011). Long-term consequences of stunting in early life. Maternal \& Child Nutrition, 7(s3), 5-18.

Deyessa, N., Berhane, Y., Alem, A., Ellsberg, M., Emmelin, M., Hogberg, U., \& Kullgren, G. (2009). Intimate partner violence and depression among women in rural Ethiopia: a cross-sectional study. Clinical Practice and Epidemiology in Mental Health, $5(1), 8$.

Dina, Y., Akintayo, J., \& Ekundayo, F.(2005). Guide to Nigerian Legal Information. Retrieved May 3, 2016 from http://www.state.gov/r/pa/ei/rls/dos/3797.htm on 23/04/2007

Dixon-Mueller, R. (1998). Female empowerment and demographic processes: Moving beyond Cairo. International Union for the Scientific Study of Population.

Dixon-Mueller, R. (2008). How young is "too young"? Comparative perspectives on adolescent sexual, marital, and reproductive transitions. Studies in Family Planning, 39(4), 247-262.

Dixon-Mueller, R. B. (2013). Rural women at work: Strategies for development in South Asia. Routledge.

Dobos, J. (2013). Analysis of Drought Conditions in the Carpathians Using Standardized Precipitation Index (SPI) 1951 - 2050. Geosciences and Engineering, 2(4), 66-80.

Doss, C. (1997). Intra-household resource allocation in Ghana: The impact of the distribution of asset ownership within the household (No. 197050). International Association of Agricultural Economists.

Drevenstedt, G. L., Crimmins, E. M., Vasunilashorn, S., \& Finch, C. E. (2008). The rise and fall of excess male infant mortality. Proceedings of the National Academy of Sciences, 105(13), 5016-5021.

Duflo, E. (2000). Child health and household resources in South Africa: evidence from the old age pension program. The American Economic Review, 90(2), 393-398.

Duflo, E. (2012). Women empowerment and economic development. Journal of Economic Literature, 50(4), 1051-1079.

Dyson, T., \& Moore, M. (1983). On kinship structure, female autonomy, and demographic behavior in India. Population and Development Review, 35-60. 
Edlund, L., \& Rahman, A. (2005). Household Structure and Child Outcomes: Nuclear vs. Extended Families. Evidence from Bangladesh. UCL and Columbia University.

Egata, G., Berhane, Y., \& Worku, A. (2014). Predictors of acute undernutrition among children aged 6 to 36 months in east rural Ethiopia: a community based nested casecontrol study. BMC Pediatrics, 14(1), 91.

Ekhator, E. O. (2015). Women and the law in Nigeria: a reappraisal. Journal of International Women's Studies, 16(2), 285.

Fantahun, M., Berhane, Y., Wall, S., Byass, P., \& Högberg, U. (2007). Women's involvement in household decision-making and strengthening social capital—crucial factors for child survival in Ethiopia. Acta Paediatrica, 96(4), 582-589.

FAO. (2016). Country Fact Sheet on Food and Agriculture Policy Trends: Bangladesh. Retrieved December, 2016, from http:/www.fao.org/3/a-i5890e.pdf

Ferreira, S., \& Ghimire, R. (2012). Forest cover, socioeconomics, and reported flood frequency in developing countries. Water Resources Research, 48(8).

Field, E., \& Ambrus, A. (2008). Early marriage, age of menarche, and female schooling attainment in Bangladesh. Journal of Political Economy, 116(5), 881-930.

Flatø, M., \& Kotsadam, A. (2014). Droughts and gender bias in infant mortality in subSaharan Africa (No. 02/2014). Memorandum, Department of Economics, University of Oslo.

Fomby, T., Ikeda, Y., \& Loayza, N. V. (2013). The growth aftermath of natural disasters. Journal of Applied Econometrics, 28(3), 412-434.

Forouzanfar, M. H., Alexander, L., Anderson, H. R., Bachman, V. F., Biryukov, S., Brauer, M., ... \& Delwiche, K. (2015). Global, regional, and national comparative risk assessment of 79 behavioural, environmental and occupational, and metabolic risks or clusters of risks in 188 countries, 1990-2013: a systematic analysis for the Global Burden of Disease Study 2013. The Lancet, 386(10010), 2287-2323.

Fotso, J. C., Cleland, J., Mberu, B., Mutua, M., \& Elungata, P. (2013). Birth spacing and child mortality: an analysis of prospective data from the Nairobi urban health and demographic surveillance system. Journal of Biosocial Science, 45(6), 779-798.

Frankenberg, E., \& Thomas, D. (2001). Measuring power. Food Consumption and Nutrition Division, International Food Policy Research Institute. 
Ghuman, S. J., Lee, H. J., \& Smith, H. L. (2006). Measurement of women's autonomy according to women and their husbands: Results from five Asian countries. Social Science Research, 35(1), 1-28.

Giddings, L., Soto, M., Rutherford, B. M., \& Maarouf, A. (2005). Standardized precipitation index zones for Mexico. Atmósfera, 18(1), 33-56.

Gorstein, J., Sullivan, K., Yip, R., De Onis, M., Trowbridge, F., Fajans, P., \& Clugston, G. (1994). Issues in the assessment of nutritional status using anthropometry. Bulletin of the World Health Organization, 72(2), 273.

Govindasamy, P., \& Malhotra, A. (1996). Women's position and family planning in Egypt. Studies in Family Planning, 328-340.

Gubhaju, B., Streatfield, K., \& Majumder, A. K. (1991). Socioeconomic, demographic and environmental determinants of infant mortality in Nepal. Journal of Biosocial Science, 23(4), 425-435.

Guerreiro, M. J., Lajinha, T., \& Abreu, I. (2008). Flood analysis with the standardized precipitation index (SPI). Revista da Faculdade de Ciência e Tecnologia, 8-14.

Guilbert, N. (2013). Early marriage, women empowerment and child mortality: Married too young to be a "good mother". Document de travall UMR DIAL.

Gupta, S. D., Mukherjee, S., Singh, S., Pande, R., \& Basu, S. (2008). Knot ready:

Lessons from India on delaying marriage for girls. Washington, DC: International Center for Research on Women.

Halder, A. K., Gurley, E. S., Naheed, A., Saha, S. K., Brooks, W. A., El Arifeen, S., ... \& Luby, S. P. (2009). Causes of early childhood deaths in urban Dhaka, Bangladesh. PLoS One, 4(12), e8145.

Hameed, W., Azmat, S. K., Ali, M., Sheikh, M. I., Abbas, G., Temmerman, M., \& Avan, B. I. (2014). Women's empowerment and contraceptive use: the role of independent versus couples' decision-making, from a lower middle income country perspective. PloS one, $9(8)$, e 104633.

Haque, M., Islam, T. M., Tareque, M. I., \& Mostofa, M. G. (2011). Women empowerment or autonomy: A comparative view in Bangladesh context. Bangladesh eJournal of Sociology, 8(2), 17-30.

Hien, N. N., \& Kam, S. (2008). Nutritional status and the characteristics related to malnutrition in children under five years of age in Nghean, Vietnam. J Prev Med Public Health, 41(4), 232-240. 
Hindin, M. J. (2000). Women's power and anthropometric status in Zimbabwe. Social Science \& Medicine, 51(10), 1517-1528.

Hindin, M. J., \& Adair, L. S. (2002). Who's at risk? Factors associated with intimate partner violence in the Philippines. Social Science \& Medicine, 55(8), 1385-1399.

Hoddinott, J., Maluccio, J. A., Behrman, J. R., Flores, R., \& Martorell, R. (2008). Effect of a nutrition intervention during early childhood on economic productivity in Guatemalan adults. The Lancet, 371(9610), 411-416.

Hossain, K. (2013). In search of equality: marriage related laws for Muslim women in Bangladesh. Journal of International Women's Studies, 5(1), 96-113.

Huffman, S. L., \& Schofield, D. (2011). Consequences of malnutrition in early life and strategies to improve maternal and child diets through targeted fortified products. Maternal \& Child Nutrition, 7(s3), 1-4.

Iannaccone, L. R. (1992). Sacrifice and stigma: Reducing free-riding in cults, communes, and other collectives. Journal of political economy, 100(2), 271-291.

Iman, A. (2003). Women, Muslim laws and human rights in Nigeria. Keynote address presented at the Woodrow Wilson International Center's Africa Program and Middle East Project on Women, Islam and Human Rights in Africa (September).

Islam, M. S., Rahaman, M. M., Aziz, K. M. S., Rahman, M., Munshi, M. H., \& Patwari, Y. (1982). Infant mortality in rural Bangladesh: an analysis of causes during neonatal and postneonatal periods. Journal of Tropical Pediatrics, 28(6), 294-298.

Jain, S., \& Kurz, K. (2007) New Insights on preventing child marriage: a global analysis of factors and programs. Washington, DC: ICRW

Jejeebhoy, S. J. (1998). Wife-beating in rural India: a husband's right? Evidence from survey data. Economic and Political Weekly, 855-862.

Jejeebhoy, S. J. (2002). Convergence and divergence in spouses' perspectives on women's autonomy in rural India. Studies in Family Planning, 33(4), 299-308.

Jensen, R., \& Oster, E. (2009). The power of TV: Cable television and women's status in India. The Quarterly Journal of Economics, 124(3), 1057-1094.

Jensen, R., \& Thornton, R. (2003). Early female marriage in the developing world. Gender \& Development, 11(2), 9-19.

Johnson, K. (2001). Media and social change: the modernizing influences of television in rural India. Media, Culture \& Society, 23(2), 147-169. 
Joseph, S., \& Nağmābādī, A. (Eds.). (2003). Encyclopedia of women and Islamic cultures: Family, law and Politics (Vol. 2). Brill Academic Publishers.

Kabir, A., Islam, M. S., Ahmed, M. S., \& Barbhuiya, K. (2001). Factors influencing infant and child mortality in Bangladesh. J Med Sci, 5, 292-5.

Kabeer, N. (1999). Resources, agency, achievements: Reflections on the measurement of women's empowerment. Development and Change, 30(3), 435-464.

Kar, B. R., Rao, S. L., \& Chandramouli, B. A. (2008). Cognitive development in children with chronic protein energy malnutrition. Behavioral and Brain Functions, 4(1), 31.

Kazianga, H., \& Udry, C. (2006). Consumption smoothing? Livestock, insurance and drought in rural Burkina Faso. Journal of Development Economics, 79(2), 413-446.

Keller, B., \& Mbewe, D. C. (1991). Policy and planning for the empowerment of Zambia's women farmers. Canadian Journal of Development Studies/Revue canadienne d'études du développement, 12(1), 75-88.

Kelly, R. J., Gray, R. H., Sewankambo, N. K., Serwadda, D., Wabwire-Mangen, F., Lutalo, T., \& Wawer, M. J. (2003). Age differences in sexual partners and risk of HIV-1 infection in rural Uganda. JAIDS Journal of Acquired Immune Deficiency Syndromes, 32(4), 446-451.

Kim, T. H. (1988). Changing determinants of infant and child mortality: on the basis of the Korean experience, 1955-73. Journal of Biosocial Science, 20(3), 345-356.

Kishor, S. (2000). Empowerment of women in Egypt and links to the survival and health of their infants. In H.Presser \& G.Sen (Eds.), Women's Empowerment and Demographic Processes: Moving Beyond Cairo. New York, NY: Oxford University Press.

Kishor, S. (2005) A Focus on Gender: Collected Papers on Gender using DHS Data. Calverton, MD: ORC Macro

Koenig, M. A., Ahmed, S., Hossain, M. B., \& Mozumder, A. B. K. A. (2003). Women's status and domestic violence in rural Bangladesh: individual-and community-level effects. Demography, 40(2), 269-288.

Kumar, D., Goel, N. K., Mittal, P. C., \& Misra, P. (2006). Influence of infant-feeding practices on nutritional status of under-five children. Indian Journal of Pediatrics, 73(5), 417-421. 
Lamidi, E. O. (2016). Multilevel analysis of state variations in women's participation in household decision-making in Nigeria. Journal of International Women's Studies, 17(1), 186.

Le Strat, Y., Dubertret, C., \& Le Foll, B. (2011). Child marriage in the United States and its association with mental health in women. Pediatrics, peds-2011.

Lee-Rife, S. M. (2010). Women's empowerment and reproductive experiences over the lifecourse. Social Science \& Medicine, 71(3), 634-642.

Lee-Rife, S., Malhotra, A., Warner, A., \& Glinski, A. M. (2012). What works to prevent child marriage: a review of the evidence. Studies in family planning, 43(4), 287-303.

Lloyd, C. B., \& Mensch, B. S. (2008). Marriage and childbirth as factors in dropping out from school: an analysis of DHS data from sub-Saharan Africa. Population Studies, 62(1), 1-13.

Loaiza Sr, E., \& Wong, S. (2012). Marrying too young. End child marriage. New York, NY: UNFPA

Lundberg, S. J., Pollak, R. A., \& Wales, T. J. (1997). Do husbands and wives pool their resources? Evidence from the United Kingdom child benefit. Journal of Human resources, 463-480

Luz, L., \& Agadjanian, V. (2015). Women's decision-making autonomy and children's schooling in rural Mozambique. Demographic Research, 32, 775.

Maitra, P. (2004). Parental bargaining, health inputs and child mortality in India. Journal of Health Economics, 23(2), 259-291.

Majumder, A. K., May, M., \& Pant, P. D. (1997). Infant and child mortality determinants in Bangladesh: are they changing?. Journal of Biosocial Science, 29(4), 385-399.

Malhotra, A. (2010). The Causes, Consequences and Solutions to Forced Child Marriage in the Developing World. International Centre for Research on Women (ICRW). New Delhi.

Malhotra, A., \& Schuler, S. R. (2005). Women's empowerment as a variable in international development. Measuring empowerment: Cross-Disciplinary Perspectives, 71-88.

Martorell, R. (1997). Undernutrition during pregnancy and early childhood and its consequences for cognitive and behavioral development. Early child development: Investing in our children's future, 39-83. 
Martorell, R., Horta, B. L., Adair, L. S., Stein, A. D., Richter, L., Fall, C. H., ... \& Victora, C. G. (2010). Weight gain in the first two years of life is an important predictor of schooling outcomes in pooled analyses from five birth cohorts from low-and middleincome countries. The Journal of Nutrition, 140(2), 348-354.

Martorell, R., \& Young, M. F. (2012). Patterns of stunting and wasting: potential explanatory factors. Advances in Nutrition: An International Review Journal, 3(2), $227-$ 233.

Mason, K. O. (1986, March). The status of women: Conceptual and methodological issues in demographic studies. In Sociological Forum (Vol. 1, No. 2, pp. 284-300). Springer Netherlands.

Matsuura, K., \& Willmott, C. (2007). Terrestrial Air Temperature and Precipitation: 1900-2006 Gridded Monthly Time Series, Version 1.01. University of Delaware. http://climate. geog. udel. edu/climate.

Mathur, S., Greene, M., \& Malhotra, A. (2003). Too young to wed: The lives, rights and health of young married girls. International Center for Research on Women

Mayor, S. (2004). Pregnancy and childbirth are leading causes of death in teenage girls in developing countries. BMJ: British Medical Journal, 328(7449), 1152.

McKee, T. B., Doesken, N. J., \& Kleist, J. (1993, January). The relationship of drought frequency and duration to time scales. In Proceedings of the 8th Conference on Applied Climatology (Vol. 17, No. 22, pp. 179-183). Boston, MA: American Meteorological Society.

McKee, T. B., Doesken, N. J., \& Kleist, J. (1995). Drought monitoring with multiple time scales. In Proceedings of the 9th Conference on Applied Climatology (pp. 233-236).

Dallas, Boston, MA: American Meteorological Society.

Miguel, E., Satyanath, S., \& Sergenti, E. (2004). Economic shocks and civil conflict: An instrumental variables approach. Journal of Political Economy, 112(4), 725-753.

Mosley, W. H., \& Chen, L. C. (1984). An analytical framework for the study of child survival in developing countries. Population and Development Review, 10(0), 25-45.

Mowla, K. (1976). Relation between climatic fluctuation and rice production in Bangladesh. In K. Takahashi \& M. Yoshino (Eds.), Climatic Change and Food Production (pp. 137-146). Tokyo: University of Tokyo Press.

Muhuri, P. K., \& Preston, S. H. (1991). Effects of family composition on mortality differentials by sex among children in Matlab, Bangladesh. The Population and Development Review, 415-434. 
Mulder, E. J., De Medina, P. R., Huizink, A. C., Van den Bergh, B. R., Buitelaar, J. K., \& Visser, G. H. (2002). Prenatal maternal stress: effects on pregnancy and the (unborn) child. Early human development, 70(1), 3-14.

Murshid, K. A. S. (1987). Weather, new technology and instability in foodgrain production in Bangladesh. The Bangladesh Development Studies, 31-56.

Myers, J. (2013) Untying the knot. Exploring early marriage in fragile states. Monrovia, California: World Vision International.

Naeye, R. L., Burt, L. S., Wright, D. L., Blanc, W. A., \& Tatter, D. (1971). Neonatal mortality, the male disadvantage. Pediatrics, 48(6), 902-906.

Nakao, T. (1996). The flood in Bangladesh and its countermeasure. Chiri (Geography), $41,38-45$.

Nguyen, M. C., \& Wodon, Q. (2012). Measuring child marriage. Economics Bulletin, 32(1), 398-411.

Nguyen, M. C., \& Wodon, Q. (2012). Estimating the impact of child marriage on literacy and education attainment in Africa. mimeo, Washington, DC: The World Bank.

Nguyen, M. C., Wodon, Q., \& Wodon, Q. (2014). Impact of child marriage on literacy and education attainment in Africa. UNICEF and UNESCO Statistics. Washington, DC: The World Bank. Retrieved from http://allinschool. org/wpcontent/uploads/2015/02/OOSC-2014-QW-Child-Marriage-final. pdf.

Nour, N. M. (2006). Health consequences of child marriage in Africa. Emerging Infectious Diseases, 12(11), 1644.

Nour, N. M. (2009). Child marriage: a silent health and human rights issue. Reviews in Obstetrics and Gynecology, 2(1), 51.

Onis, M., Dewey, K. G., Borghi, E., Onyango, A. W., Blössner, M., Daelmans, B., ... \& Branca, F. (2013). The World Health Organization's global target for reducing childhood stunting by 2025: rationale and proposed actions. Maternal \& Child Nutrition, 9(S2), 626.

Ostien, P. (2007) Nigeria's Sharia Criminal Codes. In P. Ostein (Ed.), Sharia Implementation in Northern Nigeria, 1996-2006: A Sourcebook. Vol 4. Ibadan: Spectrum Books Ltd.

Parsons, J., Edmeades, J., Kes, A., Petroni, S., Sexton, M., \& Wodon, Q. (2015). Economic impacts of child marriage: A review of the literature. The Review of Faith \& International Affairs, 13(3), 12-22. 
Paltineanu, C. R., Mihailescu, I. F., Prefac, Z., Dragota, C., Vasenciuc, F., \& Claudia, N. (2009). Combining the standardized precipitation index and climatic water deficit in characterizing droughts: a case study in Romania. Theoretical and Applied Climatology, 97(3-4), 219.

Paul, B. K., \& Rasid, H. (1993). Flood damage to rice crop in Bangladesh. Geographical Review, 150-159.

Pebley, A. R., \& Millman, S. (1986). Birthspacing and child survival. International Family Planning Perspectives, 71-79.

Pervin, S., Khan, M. A., \& Shah, M. M. H. (2014). Household-level Analysis of Women's Power Practice in Old Dhaka City, Bangladesh. Journal of Comparative Asian Development, 13(1), 174-203.

Peters, R. (2005). Crime and punishment in Islamic law: theory and practice from the sixteenth to the twenty-first century (No. 2). Cambridge, UK: Cambridge University Press.

Pongou, R. (2013). Why is infant mortality higher in boys than in girls? A new hypothesis based on preconception environment and evidence from a large sample of twins. Demography, 50(2), 421-444.

Pozarny, P. (2016). Gender roles and opportunities for women in urban environments. Retrieved from http://www.gsdrc.org/wp-content/uploads/2016/01/HDQ1337.pdf

Prendergast, A. J., \& Humphrey, J. H. (2014). The stunting syndrome in developing countries. Paediatrics and International Child Health, 34(4), 250-265.

Quinlivan, J. (2000). Study of adolescent pregnancy in Western Australia: The way forward. Children, young people and domestic violence. Melbourne, Australia: Partnerships Against Domestic Violence.

Quisumbing, A. R., \& Maluccio, J. A. (2000). Intrahousehold allocation and gender relations: New empirical evidence from four developing countries. Washington, DC: International Food Policy Research Institute.

Rafferty, Y. (2013). International dimensions of discrimination and violence against girls: A human rights perspective. Journal of International Women's Studies, 14(1), 1.

Raj, A., Saggurti, N., Balaiah, D., \& Silverman, J. G. (2009). Prevalence of child marriage and its effect on fertility and fertility-control outcomes of young women in India: a cross-sectional, observational study. The Lancet, 373(9678), 1883-1889. 
Raj, A., Saggurti, N., Winter, M., Labonte, A., Decker, M. R., Balaiah, D., \& Silverman, J. G. (2010). The effect of maternal child marriage on morbidity and mortality of children under 5 in India: cross sectional study of a nationally representative sample. BMJ, 340, b4258.

Raj, A. (2010). When the mother is a child: the impact of child marriage on the health and human rights of girls. Archives of disease in childhood, 95, 931-935.

Rahman, M., Hoque, M. A., Mostofa, M. G., \& Makinoda, S. (2014). Association between adolescent marriage and intimate partner violence: a study of young adult women in Bangladesh. Asia Pacific Journal of Public Health, 26(2), 160-168.

Ramalingaswami, V., Jonsson, U., \& Rohde, J. (1997). Malnutrition: a South Asian enigma. In S. Gillespie (Ed.), Malnutrition in South Asia: a regional profile (pp. 11-17). New York, NY: UNICEF

Rana, M. S., \& Islam, M. R. (2015). Impact of Flood Hazards on the Agricultural Production and Livelihood Shifting in Rural Bangladesh: A Comparative Study. Retrieved from http://www.academia.edu/download/37583665/Impact_of_Flood_Hazards_on the_Agric ultural_Production_and_Livelihood_Shifting_in_Rural_Bangladesh_A_Comparative_St udy.pdf.

Ray, S. (2013). Relationship between stunting and wasting in children. Medical Journal Armed Forces India, 69(4), 410.

Rayhan, M. I., \& Khan, M. S. H. (2006). Factors causing malnutrition among under five children in Bangladesh. Pak J Nutr, 5(6), 558-562.

Read, J. S., Troendle, J. F., \& Klebanoff, M. A. (1997). Infectious disease mortality among infants in the United States, 1983 through 1987. American Journal of Public Health, 87(2), 192-198.

Rodríguez, L. (2016). Intrahousehold Inequalities in Child Rights and Well-Being. A Barrier to Progress?. World Development, 83, 111-134.

Rosales-Rueda, M. F. (2014). Family investment responses to childhood health conditions: Intrafamily allocation of resources. Journal of Health Economics, 37, 41-57.

Rosales-Rueda, M. (2014). Impact of early life shocks on human capital formation: Evidence from el nino floods in Ecuador. University of California Irvine working paper.

Rosenzweig, M. R., \& Wolpin, K. I. (1994). Are there increasing returns to the intergenerational production of human capital? Maternal schooling and child intellectual achievement. Journal of Human Resources, 670-693. 
Ross, S. D. (2009). Women's human rights: the international and comparative law casebook. University of Pennsylvania Press.

Ruel, M. T., Alderman, H., \& Maternal and Child Nutrition Study Group. (2013). Nutrition-sensitive interventions and programmes: how can they help to accelerate progress in improving maternal and child nutrition?. The Lancet, 382(9891), 536-551.

Santhya, K. G., Haberland, N., Ram, F., Sinha, R. K., \& Mohanty, S. K. (2007). Consent and coercion: examining unwanted sex among married young women in India. International Family Planning Perspectives, 124-132.

Save the Children. (2013). Surviving the First Day: State of the World's Mothers 2013. Retrieved from http://www.savethechildren.org/atf/cf/\%7B9def2ebe-10ae-432c-9bd0df91d2eba74a\%7D/SOWM-FULL-REPORT 2013.PDF.

Schultz, T. P. (1990). Testing the neoclassical model of family labor supply and fertility. Journal of Human resources, 599-634.

Sharghi, A., Kamran, A., \& Faridan, M. (2011). Evaluating risk factors for proteinenergy malnutrition in children under the age of six years: a case-control study from Iran. International Journal of General Medicine, 4, 607-611.

Shroff, M., Griffiths, P., Adair, L., Suchindran, C., \& Bentley, M. (2009). Maternal autonomy is inversely related to child stunting in Andhra Pradesh, India. Maternal \& Child Nutrition, 5(1), 64-74.

Shroff, M. R., Griffiths, P. L., Suchindran, C., Nagalla, B., Vazir, S., \& Bentley, M. E. (2011). Does maternal autonomy influence feeding practices and infant growth in rural India?. Social Science \& Medicine, 73(3), 447-455.

Smith, L. C., \& Haddad, L. (2015). Reducing child undernutrition: past drivers and priorities for the post-MDG era. World Development, 68, 180-204.

Smith, L. C., Ruel, M. T., \& Ndiaye, A. (2005). Why is child malnutrition lower in urban than in rural areas? Evidence from 36 developing countries. World Development, 33(8), 1285-1305.

Smith, L., Ramakrishnan, U., Ndiaye, A., Haddad, L., \& Matrorell, R. The importance of women's status for child nutrition in developing countries (2003). IFPRI Reports, 131.

Stone, L. (2012). Ending child marriage and meeting the needs of married children: the USAID vision for action. Retrieved from http://pdf.usaid.gov/pdf_docs/PDACU300.pdf. 
Stromquist, N. P. (2014). Women in the Third World: An encyclopedia of contemporary Issues. New York,NY: Routledge.

Tertsakian, C. (2004). Nigeria" Political Shari'a"?: Human Rights and Islamic Law in Northern Nigeria (Vol. 16, No. 9). Human Rights Watch.

Tertilt, M., \& Doepke, M. (2010). Does Female Empowerment Promote Economic Development?. In 2010 Meeting Papers (No. 230). Society for Economic Dynamics.

Thomas, D. (1990). Intra-household resource allocation: An inferential approach. Journal of Human Resources, 635-664.

Thomas, D., \& Strauss, J. (1997). Health and wages: Evidence on men and women in urban Brazil. Journal of Econometrics, 77(1), 159-185.

UNICEF. (2007). Progress for children: a world fit for children statistical review (No. 6). New York, NY: UNICEF

UNICEF. (2014). Hidden in plain sight: A statistical analysis of violence against children. New York, NY: UNICEF

UNICEF. (2015). Levels \& Trends in Child Mortality. Retrieved from http://www.childmortality.org/files_v20/download/IGME\%20Report\%202015_9_3\%20L R\%20Web.pdf

UNICEF. (2016). State of the World's Children. New York, NY: UNICEF

Union, A. (2015). The Effects of Traditional and Religious Practices of Child Marriage on Africa's Socio-Economic Development. Retrieved from https://static1.squarespace.com/static/5519047ce4b0d9aaa8c82e69/t/5746d78e2b8dde64f dce077f/1464260671406/Study+on+Tradition_+Religion_Childmarriage_Africa_report.p df

Upadhyay, U. D., \& Hindin, M. J. (2005). Do higher status and more autonomous women have longer birth intervals? : Results from Cebu, Philippines. Social Science \& Medicine, $60(11), 2641-2655$.

Van Poppel, F. W. (1979). Regional differences in mortality in Western and Northern Europe: a review of the situation in the seventies.

Veghari, G. R. (2007). Assessment of physical growth among the under 6 years children in rural area in Gorgan, Iran. Pak J Nutr, 6(3), 252-255.

Verma, R., Sinha, T., \& Khanna, T. (2013). Asia Child Marriage Initiative: Summary of research in Bangladesh India and Nepal. Bangkok, Thailand: Plan Asia Regional Office 
Vitolo, M. R., Gama, C. M., Bortolini, G. A., Campagnolo, P. D., \& Drachler, M. D. L. (2008). Some risk factors associated with overweight, stunting and wasting among children under 5 years old. Jornal De Pediatria, 84(3), 251-257.

Vogelstein, R. B. (2013). Ending child marriage: How elevating the status of girls advances US foreign policy objectives. New York, NY: Council on Foreign Relations.

Waldron, I. (1983). Sex differences in human mortality: the role of genetic factors. Social Science \& Medicine, 17(6), 321-333.

Waldron, I. (1985). What do we know about causes of sex differences in mortality? A review of the literature. Population Bulletin of the United Nations, (18), 59-76.

Walker, S. P., Chang, S. M., Powell, C. A., Simonoff, E., \& Grantham-McGregor, S. M. (2007). Early childhood stunting is associated with poor psychological functioning in late adolescence and effects are reduced by psychosocial stimulation. The Journal of Nutrition, 137(11), 2464-2469.

WHO Working Group. (1986). Use and interpretation of anthropometric indicators of nutritional status. Bulletin of the World Health Organization, 64(6), 929.

WHO. (1995). Physical status: The use of and interpretation of anthropometry, Report of a WHO Expert Committee. Geneva, Switzerland: World Health Organization.

WHO. (2006). WHO child growth standards: length/height for age, weight-for-age, weight-for-length, weight-for-height and body mass index-for-age, methods and development. Geneva, Switzerland: World Health Organization.

WHO. (2010). Nutrition Landscape Information System (NLIS) country profile indicators: interpretation guide. Geneva, Switzerland: World Health Organization

WHO. (2013). Childhood Stunting: Context, Causes, Consequences. Geneva, Switzerland: World Health Organization.

WMO. (2006). Social aspects and stakeholder involvement in integrated flood management: Associated Programme on Flood Management No.1008 


\section{APPENDIX}

Table A1: Correlations

Child Marriage

\begin{tabular}{lc}
\hline Child Marriage & 1 \\
Urban & 0.193 \\
Mother Primary & 0.217 \\
Mother Literate & 0.334 \\
Father Primary & 0.265 \\
Lowest Quintile & 0.204 \\
Second Quintile & 0.171 \\
Fourth Quintile & 0.112 \\
Highest Quintile & 0.330 \\
Teenage Pregnancy & 0.662 \\
\hline
\end{tabular}


Table A2: OLS Results - Effect on Moderate Stunting and Severe Stunting

\begin{tabular}{|c|c|c|c|c|}
\hline & $\begin{array}{c}\text { (1) } \\
\text { Moderate Stunting } \\
\text { OLS }\end{array}$ & $\begin{array}{c}\text { (2) } \\
\text { Moderate Stunting } \\
\text { OLS }\end{array}$ & $\begin{array}{c}\text { (3) } \\
\text { Severe Stunting } \\
\text { OLS }\end{array}$ & $\begin{array}{c}\text { (4) } \\
\text { Severe Stunting } \\
\text { OLS }\end{array}$ \\
\hline Child Marriage & $\begin{array}{c}0.00432 \\
(0.00592)\end{array}$ & $\begin{array}{c}0.00736 \\
(0.00750)\end{array}$ & $\begin{array}{l}0.0124 * * \\
(0.00506)\end{array}$ & $\begin{array}{c}0.00635 \\
(0.00640)\end{array}$ \\
\hline Urban & $\begin{array}{l}-0.0155 \\
(0.0286)\end{array}$ & $\begin{array}{l}-0.0155 \\
(0.0286)\end{array}$ & $\begin{array}{r}-0.00291 \\
(0.0244)\end{array}$ & $\begin{array}{r}-0.00299 \\
(0.0244)\end{array}$ \\
\hline Mother Primary Edu & $\begin{array}{l}0.00152 \\
(0.0336)\end{array}$ & $\begin{array}{l}0.00262 \\
(0.0336)\end{array}$ & $\begin{array}{l}0.00424 \\
(0.0287)\end{array}$ & $\begin{array}{l}0.00207 \\
(0.0287)\end{array}$ \\
\hline Mother Literate & $\begin{array}{l}0.00984 \\
(0.0326)\end{array}$ & $\begin{array}{l}0.00997 \\
(0.0326)\end{array}$ & $\begin{array}{c}-0.0605 * * \\
(0.0278)\end{array}$ & $\begin{array}{c}-0.0607 * * \\
(0.0278)\end{array}$ \\
\hline Father Primary Edu & $\begin{array}{c}-0.0704 * * \\
(0.0282)\end{array}$ & $\begin{array}{c}-0.0698 * * \\
(0.0282)\end{array}$ & $\begin{array}{c}-0.0447 * \\
(0.0241)\end{array}$ & $\begin{array}{c}-0.0459 * \\
(0.0241)\end{array}$ \\
\hline Lowest Quintile & $\begin{array}{l}-0.0575 \\
(0.0386)\end{array}$ & $\begin{array}{c}-0.0573 \\
(0.0386)\end{array}$ & $\begin{array}{l}0.0586^{*} \\
(0.0330)\end{array}$ & $\begin{array}{l}0.0583 * \\
(0.0330)\end{array}$ \\
\hline Second Quintile & $\begin{array}{l}-0.0283 \\
(0.0386)\end{array}$ & $\begin{array}{l}-0.0278 \\
(0.0386)\end{array}$ & $\begin{array}{l}-0.0126 \\
(0.0330)\end{array}$ & $\begin{array}{l}-0.0135 \\
(0.0330)\end{array}$ \\
\hline Fourth Quintile & $\begin{array}{c}-0.0921 * * \\
(0.0378)\end{array}$ & $\begin{array}{c}-0.0937 * * \\
(0.0379)\end{array}$ & $\begin{array}{r}-0.00341 \\
(0.0323)\end{array}$ & $\begin{array}{c}-0.000169 \\
(0.0324)\end{array}$ \\
\hline Highest Quintile & $\begin{array}{c}-0.121 * * * \\
(0.0398)\end{array}$ & $\begin{array}{c}-0.123 * * * \\
(0.0400)\end{array}$ & $\begin{array}{c}-0.0684 * * \\
(0.0340)\end{array}$ & $\begin{array}{c}-0.0643 * \\
(0.0341)\end{array}$ \\
\hline Flood Shock at Birth & $\begin{array}{c}-0.00397 \\
(0.262)\end{array}$ & $\begin{array}{c}-0.00646 \\
(0.262)\end{array}$ & $\begin{array}{c}-0.467 * * \\
(0.224)\end{array}$ & $\begin{array}{c}-0.462 * * \\
(0.224)\end{array}$ \\
\hline Teenage Pregnancy & & $\begin{array}{l}-0.0202 \\
(0.0305)\end{array}$ & & $\begin{array}{c}0.0399 \\
(0.0261)\end{array}$ \\
\hline $\mathrm{FE}$ & yes & Yes & yes & yes \\
\hline $\mathrm{R} 2$ & 0.0226 & 0.0229 & 0.0545 & 0.0560 \\
\hline Observations & 1555 & 1555 & 1555 & 1555 \\
\hline
\end{tabular}

Standard errors in parentheses

1999-2000 DHS Data. OLS regression; dependent variable is children's health outcomes measured by moderate stunting (Columns 1 to 2 ) severe stunting (Columns 3 to 4 ).

$* \mathrm{p}<0.10, * * \mathrm{p}<0.05, * * * \mathrm{p}<0.010$ 
Table A3: First-stage IV Results - Child Mortality by Gender

\begin{tabular}{|c|c|c|c|c|}
\hline & $\begin{array}{c}\text { (1) } \\
\text { Child Marriage }\end{array}$ & $\begin{array}{c}\text { (2) } \\
\text { Child Marriage }\end{array}$ & $\begin{array}{c}(3) \\
\text { Child Marriage }\end{array}$ & $\begin{array}{c}\text { (4) } \\
\text { Child Marriage }\end{array}$ \\
\hline FS -12 to 13 & $\begin{array}{l}-0.0324^{* * *} \\
(0.0118)\end{array}$ & $\begin{array}{l}-0.0425^{* * *} \\
(0.0118)\end{array}$ & $\begin{array}{l}-0.0103 \\
(0.0122)\end{array}$ & $\begin{array}{l}-0.0186 \\
(0.0122)\end{array}$ \\
\hline FS -14 to 15 & $\begin{array}{l}0.00666 \\
(0.0117)\end{array}$ & $\begin{array}{l}-0.00887 \\
(0.0118)\end{array}$ & $\begin{array}{l}-0.0207^{*} \\
(0.0120)\end{array}$ & $\begin{array}{c}-0.0344^{* * *} \\
(0.0121)\end{array}$ \\
\hline FS -16 to 17 & $\begin{array}{l}-0.0225^{* *} \\
(0.0114)\end{array}$ & $\begin{array}{c}-0.0434^{* * *} \\
(0.0116)\end{array}$ & $\begin{array}{l}-0.0206^{*} \\
(0.0114)\end{array}$ & $\begin{array}{c}-0.0361^{* * *} \\
(0.0115)\end{array}$ \\
\hline Age at Birth & $\begin{array}{l}-0.0112^{* * *} \\
(0.000665)\end{array}$ & $\begin{array}{l}-0.0109^{* * *} \\
(0.000661)\end{array}$ & $\begin{array}{l}-0.0115^{* * *} \\
(0.000682)\end{array}$ & $\begin{array}{l}-0.0109^{* * *} \\
(0.000681)\end{array}$ \\
\hline Highest Quintile & $\begin{array}{c}-0.0858^{* * *} \\
(0.0104)\end{array}$ & $\begin{array}{l}-0.0767^{* * *} \\
(0.0104)\end{array}$ & $\begin{array}{c}-0.0607^{* * *} \\
(0.0105)\end{array}$ & $\begin{array}{l}-0.0512^{* * *} \\
(0.0105)\end{array}$ \\
\hline Fourth Quintile & $\begin{array}{l}-0.0159^{*} \\
(0.00965)\end{array}$ & $\begin{array}{l}-0.00859 \\
(0.00959)\end{array}$ & $\begin{array}{c}-0.0127 \\
(0.00991)\end{array}$ & $\begin{array}{l}-0.00781 \\
(0.00985)\end{array}$ \\
\hline Lowest Quintile & $\begin{array}{l}-0.0271^{* * *} \\
(0.0102)\end{array}$ & $\begin{array}{l}-0.0196^{*} \\
(0.0102)\end{array}$ & $\begin{array}{l}-0.0218^{* *} \\
(0.0103)\end{array}$ & $\begin{array}{l}-0.0151 \\
(0.0103)\end{array}$ \\
\hline Second Quintile & $\begin{array}{l}-0.0223^{* *} \\
(0.00982)\end{array}$ & $\begin{array}{l}-0.0217^{* *} \\
(0.00974)\end{array}$ & $\begin{array}{l}-0.00605 \\
(0.0100)\end{array}$ & $\begin{array}{l}-0.00666 \\
(0.00997)\end{array}$ \\
\hline Father-Primary Edu & $\begin{array}{l}-0.0297^{* * *} \\
(0.00716)\end{array}$ & $\begin{array}{l}-0.0288^{* * *} \\
(0.00712)\end{array}$ & $\begin{array}{l}-0.0372^{* * *} \\
(0.00718)\end{array}$ & $\begin{array}{l}-0.0377^{* * *} \\
(0.00714)\end{array}$ \\
\hline Mother-Primary Edu & $\begin{array}{l}-0.0708^{* * *} \\
(0.00717)\end{array}$ & $\begin{array}{l}-0.0793^{* * *} \\
(0.00721)\end{array}$ & $\begin{array}{l}-0.0633^{* * *} \\
(0.00728)\end{array}$ & $\begin{array}{l}-0.0712^{* * *} \\
(0.00732)\end{array}$ \\
\hline Urban & $\begin{array}{l}-0.0272^{* * *} \\
(0.00779)\end{array}$ & $\begin{array}{l}-0.0259^{* * *} \\
(0.00776)\end{array}$ & $\begin{array}{l}-0.0506^{* * *} \\
(0.00795)\end{array}$ & $\begin{array}{l}-0.0500^{* * *} \\
(0.00792)\end{array}$ \\
\hline Nuclear Family & $\begin{array}{c}0.00680 \\
(0.00747)\end{array}$ & $\begin{array}{l}-0.00260 \\
(0.00745)\end{array}$ & $\begin{array}{c}-0.00354 \\
(0.00767)\end{array}$ & $\begin{array}{l}-0.00958 \\
(0.00764)\end{array}$ \\
\hline Living Children in $\mathrm{HH}$ & $\begin{array}{l}0.0537^{* * *} \\
(0.00287)\end{array}$ & $\begin{array}{l}0.0534^{* * *} \\
(0.00286)\end{array}$ & $\begin{array}{c}0.0539^{* * *} \\
(0.00298)\end{array}$ & $\begin{array}{l}0.0528^{* * *} \\
(0.00297)\end{array}$ \\
\hline Birth Interval & $\begin{array}{c}0.00217 \\
(0.00960) \\
\end{array}$ & $\begin{array}{c}0.00456 \\
(0.00952) \\
\end{array}$ & $\begin{array}{r}-0.000545 \\
(0.00990) \\
\end{array}$ & $\begin{array}{c}0.00431 \\
(0.00983) \\
\end{array}$ \\
\hline $\begin{array}{l}\text { FE } \\
\text { F-Stat } \\
\text { R2 } \\
\text { Observations }\end{array}$ & $\begin{array}{c}\text { no } \\
65.87 \\
0.0797 \\
10664\end{array}$ & $\begin{array}{c}\text { yes } \\
66.80 \\
0.0808 \\
10664\end{array}$ & $\begin{array}{c}\text { yes } \\
62.31 \\
0.0785 \\
10255\end{array}$ & $\begin{array}{c}\text { yes } \\
62.19 \\
0.0784 \\
10255\end{array}$ \\
\hline
\end{tabular}

Standard errors in parentheses

OLS

${ }^{*} p<.1,{ }^{* *} p<.05,{ }^{* * *} p<.01$ 
Table A4: First-stage IV Results - Infant Mortality by Gender

\begin{tabular}{|c|c|c|c|c|}
\hline & $\begin{array}{c}\text { (1) } \\
\text { Child Marriage }\end{array}$ & $\begin{array}{c}\text { (2) } \\
\text { Child Marriage }\end{array}$ & $\begin{array}{c}\text { (3) } \\
\text { Child Marriage }\end{array}$ & $\begin{array}{c}\text { (4) } \\
\text { Child Marriage }\end{array}$ \\
\hline FS -12 to 13 & $\begin{array}{l}-0.0387^{* * *} \\
(0.0110)\end{array}$ & $\begin{array}{l}-0.0496^{* * *} \\
(0.0110)\end{array}$ & $\begin{array}{c}-0.0336^{* * *} \\
(0.0114)\end{array}$ & $\begin{array}{l}-0.0436^{* * *} \\
(0.0114)\end{array}$ \\
\hline FS -14 to 15 & $\begin{array}{l}0.00128 \\
(0.0110)\end{array}$ & $\begin{array}{l}-0.0130 \\
(0.0111)\end{array}$ & $\begin{array}{l}-0.0269^{* *} \\
(0.0114)\end{array}$ & $\begin{array}{c}-0.0389^{* * *} \\
(0.0115)\end{array}$ \\
\hline FS - 16 to 17 & $\begin{array}{l}-0.0176 \\
(0.0109)\end{array}$ & $\begin{array}{l}-0.0358^{* * *} \\
(0.0110)\end{array}$ & $\begin{array}{l}-0.0156 \\
(0.0110)\end{array}$ & $\begin{array}{c}-0.0307^{* * *} \\
(0.0110)\end{array}$ \\
\hline Age at Birth & $\begin{array}{l}-0.0118^{* * *} \\
(0.000609)\end{array}$ & $\begin{array}{l}-0.0114^{* * *} \\
(0.000607)\end{array}$ & $\begin{array}{l}-0.0132^{* * *} \\
(0.000636)\end{array}$ & $\begin{array}{l}-0.0124^{* * *} \\
(0.000635)\end{array}$ \\
\hline Highest Quintile & $\begin{array}{l}-0.0909^{* * *} \\
(0.00991)\end{array}$ & $\begin{array}{l}-0.0822^{* * *} \\
(0.00992)\end{array}$ & $\begin{array}{c}-0.0618^{* * *} \\
(0.0101)\end{array}$ & $\begin{array}{l}-0.0526^{* * *} \\
(0.0101)\end{array}$ \\
\hline Fourth Quintile & $\begin{array}{l}-0.0186^{* *} \\
(0.00923)\end{array}$ & $\begin{array}{c}-0.0114 \\
(0.00917)\end{array}$ & $\begin{array}{l}-0.00392 \\
(0.00950)\end{array}$ & $\begin{array}{c}0.00132 \\
(0.00944)\end{array}$ \\
\hline Lowest Quintile & $\begin{array}{l}-0.0297^{* * *} \\
(0.00960)\end{array}$ & $\begin{array}{l}-0.0221^{* *} \\
(0.00955)\end{array}$ & $\begin{array}{c}-0.0112 \\
(0.00975)\end{array}$ & $\begin{array}{l}-0.00483 \\
(0.00970)\end{array}$ \\
\hline Second Quintile & $\begin{array}{l}-0.0254^{* * *} \\
(0.00931)\end{array}$ & $\begin{array}{l}-0.0247^{* * *} \\
(0.00924)\end{array}$ & $\begin{array}{c}0.00871 \\
(0.00956)\end{array}$ & $\begin{array}{c}0.00775 \\
(0.00950)\end{array}$ \\
\hline Father-Primary Edu & $\begin{array}{l}-0.0372^{* * *} \\
(0.00678)\end{array}$ & $\begin{array}{l}-0.0359^{* * *} \\
(0.00676)\end{array}$ & $\begin{array}{l}-0.0369^{* * *} \\
(0.00686)\end{array}$ & $\begin{array}{l}-0.0379^{* * *} \\
(0.00683)\end{array}$ \\
\hline Mother-Primary Edu & $\begin{array}{l}-0.0798^{* * *} \\
(0.00679)\end{array}$ & $\begin{array}{l}-0.0884^{* * *} \\
(0.00682)\end{array}$ & $\begin{array}{l}-0.0772^{* * *} \\
(0.00692)\end{array}$ & $\begin{array}{l}-0.0856^{* * *} \\
(0.00695)\end{array}$ \\
\hline urban & $\begin{array}{l}-0.0294^{* * *} \\
(0.00739)\end{array}$ & $\begin{array}{l}-0.0290^{* * *} \\
(0.00737)\end{array}$ & $\begin{array}{l}-0.0472^{* * *} \\
(0.00759)\end{array}$ & $\begin{array}{l}-0.0466^{* * *} \\
(0.00756)\end{array}$ \\
\hline Nuclear Family & $\begin{array}{l}0.0298^{* * *} \\
(0.00696)\end{array}$ & $\begin{array}{l}0.0214^{* * *} \\
(0.00694)\end{array}$ & $\begin{array}{l}0.0266^{* * *} \\
(0.00718)\end{array}$ & $\begin{array}{l}0.0201^{* * *} \\
(0.00715)\end{array}$ \\
\hline Living Children in $\mathrm{HH}$ & $\begin{array}{l}0.0574^{* * *} \\
(0.00269)\end{array}$ & $\begin{array}{c}0.0577^{* * *} \\
(0.00268)\end{array}$ & $\begin{array}{l}0.0605^{* * *} \\
(0.00278)\end{array}$ & $\begin{array}{c}0.0599^{* * *} \\
(0.00278)\end{array}$ \\
\hline Birth Interval & $\begin{array}{c}0.00551 \\
(0.00947)\end{array}$ & $\begin{array}{c}0.00853 \\
(0.00940)\end{array}$ & $\begin{array}{c}0.0000804 \\
(0.00977)\end{array}$ & $\begin{array}{c}0.00471 \\
(0.00970)\end{array}$ \\
\hline FE & no & yes & no & yes \\
\hline F-Stat & 92.21 & 93.49 & 92.58 & 92.69 \\
\hline $\mathrm{R} 2$ & 0.0903 & 0.0915 & 0.0941 & 0.0942 \\
\hline Observations & 13017 & 13017 & 12494 & 12494 \\
\hline
\end{tabular}


VITA

\section{AMY VIJAYA RAMNARINE}

2012

B.S., Mathematics

Florida A\&M University

Tallahassee, Florida

2014

M.A., Economics

Florida International University

Miami, Florida

$2012-2017$

Graduate Teaching Assistant and Doctoral Student

Florida International University

Miami, Florida

2017

Joins Occidental College faculty in August

Occidental College

Los Angeles, California

\section{PRESENTATIONS}

The Northeast Universities Development Consortium Conference, Fall 2017, Medford, MA. Presented "The Effect of Child Marriage on Children's Health Outcomes: Evidence from Bangladesh."

Midwest Economics Association 2017 Annual Meeting, Spring 2017, Cincinnati, OH. Presented "The Effect of Child Marriage on Children's Health Outcomes: Evidence from Bangladesh."

86th Southern Economic Association Conference, Fall 2016, Washington, DC. Presented "The Effect of Child Marriage on Children's Health Outcomes: Evidence from Bangladesh."

42nd Eastern Economic Association Conference, Spring 2016, Washington, DC. Presented "The Effect of Child Marriage on Children's Health Outcomes: Evidence from Bangladesh." 